\title{
Optical analysis of synaptic vesicle protein molecules during exo- and endocytosis using $\mathrm{pH}$-switchable fluorescent probes
}

\author{
PhD Thesis \\ in partial fulfillment of the requirements \\ for the degree "Doctor of Philosophy (PhD)" \\ in the Neuroscience Program \\ at the Georg August University Göttingen, \\ Faculty of Biology
}

Submitted by

Raunak Sinha

Born in

Kolkata, India 


\section{DECLARATION}

I hereby declare that my $\mathrm{PhD}$ thesis 'Optical analysis of synaptic vesicle protein molecules during exo- and endocytosis using $\mathrm{pH}$-switchable fluorescent probes' has been written independently with no other aids or sources than quoted.

Raunak Sinha 
To my grandfather Tarani Kumar Datta

for his love, support and motivation. 


\section{TABLE OF CONTENTS}

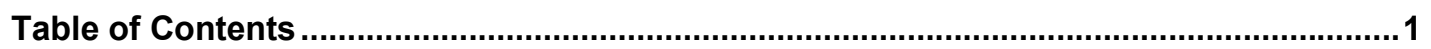

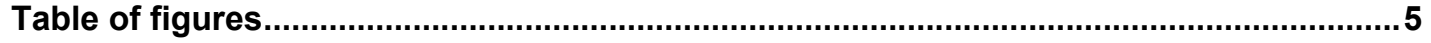

Abstract

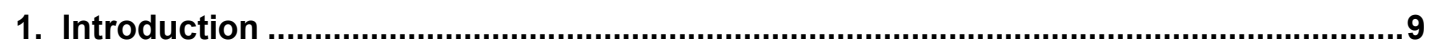

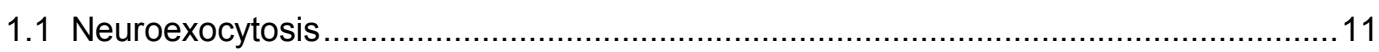

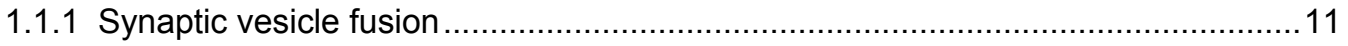

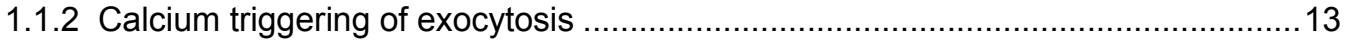

1.1.3 Minimal fusion machinery; SNAREs …….........................................................

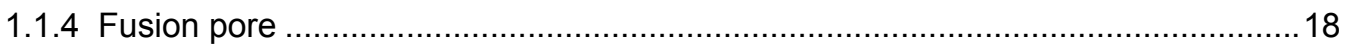

1.1.5 Minimal number of SNARE complexes necessary for fusion ................................19

1.1.6 Copy numbers of synaptic vesicle proteins ....................................................22

1.1.7 Visualizing SNARE molecules during single vesicle fusion ................................24

1.1.8 Single molecule optical methods to determine protein copy numbers....................25

1.2 Synaptic vesicle recycling; different forms of endocytosis ......................................28

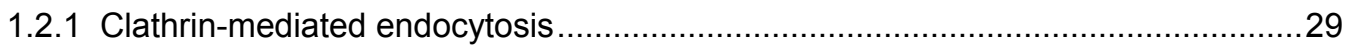

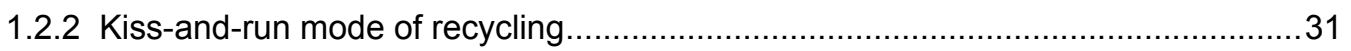

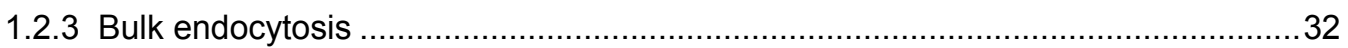

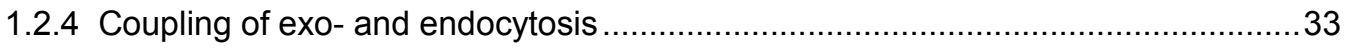

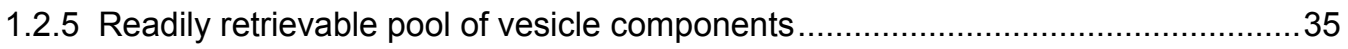

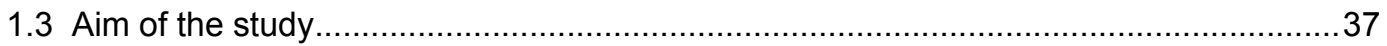

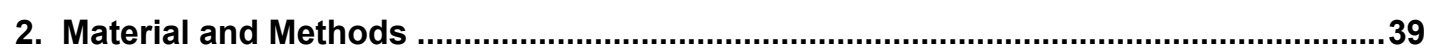

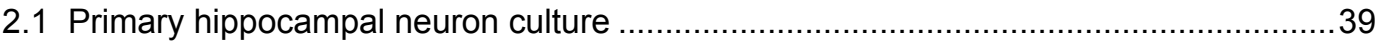

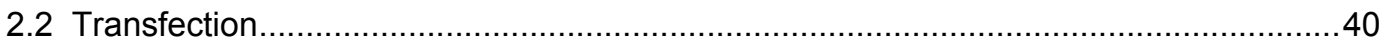

2.3 Synaptobrevin 2/ Cellubrevin mutant mice .......................................................... 41

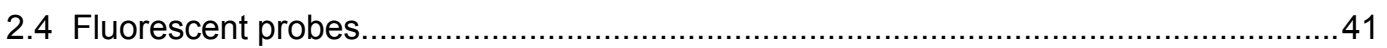

2.4.1 Overexpression of pHluorin-tagged SV proteins ............................................ 42

2.4.2 Live-cell immunolabeling with cypHer5E-coupled antibodies ..............................45 
2.5 Tobacco etch virus protease cleavage

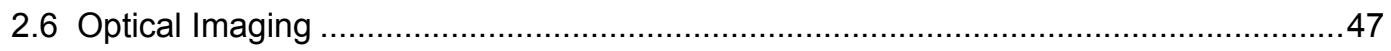

2.7 Single vesicle optical recordings with pHluorin-based probes .................................47

2.8 pHluorin and cypHer measurements (bulk stimulation) .....................................48

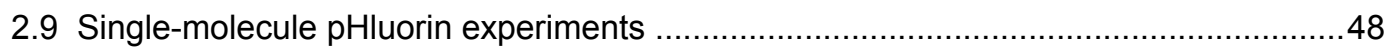

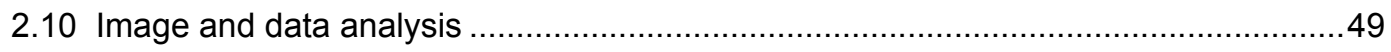

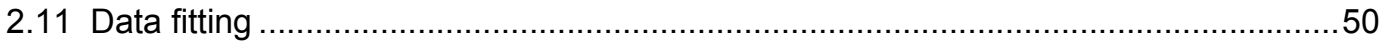

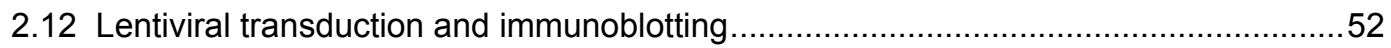

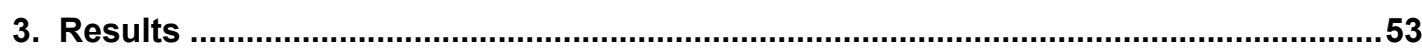

3.1 Number of SNARE complexes necessary and sufficient for evoked fusion ................53

3.1.1 Optical recording of single vesicle fusion ............................................. 53

3.1.2 Single action potential fluorescence responses display quantal distribution .........57

3.1.3 Quantal size is equivalent to single pHluorin molecule fluorescence ..................59

3.1.3a Single molecule bleaching analysis of immobilized pHluorin..............................59

3.1.3b Single molecule bleaching analysis of pHluorin in neuronal measurements ........61

3.1.4 Average number of pHluorin reporters targeted per synaptic vesicle .................63

3.1.5 SynaptopHluorin rescues $\mathrm{Ca}^{2+}$-evoked release in Syb2/Ceb null hippocampal neurons

3.1.6 Two copies of SynaptopHluorin are necessary and sufficient for evoked SV fusion 68

3.1.7 Single SynaptopHluorin molecule is unable to drive evoked fusion ....................70

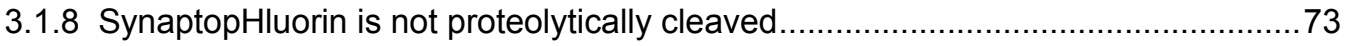

3.2 Number of Synaptotagmin1 molecules required to trigger fusion ............................75

3.3 Spatial and temporal kinetics of the readily retrievable pool of native vesicle proteins.79

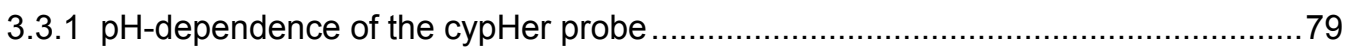

3.3.2 Live-cell labeling of neurons with cypHer-coupled antibodies ........................... 80

3.3.3 Stimulation-dependent exo-endocytosis measured using cypHer-coupled antibodies

3.3.4 Size of the surface pool of synaptic vesicle constituents.

3.3.5 Dual-color measurements of vesicle recycling kinetics using SynaptopHluorin and cypHer-coupled antibodies.............................................................................. 86

3.3.6 Kinetics of the readily retrievable surface pool of synaptic vesicle constituents.....88

3.3.7 Size of the readily retrievable pool compared to the readily releasable pool .........91 
4.1 Two SNARE complexes are sufficient for vesicle fusion during fast synaptic

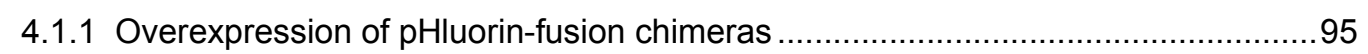

4.1.2 Regulation of synaptic vesicle protein stochiometry and release probability..........96

4.1.3 pHluorin maturation efficiency; implication for fluorescence amplitude estimation.98

4.1.4 Effects of local environment on pHluorin fluorescence ................................99

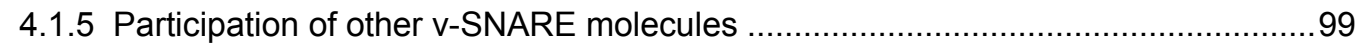

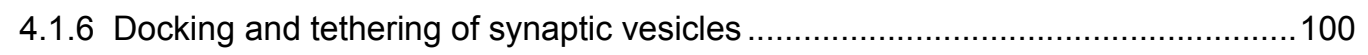

4.1.7 Molecular nature of the fusion pore ............................................................. 101

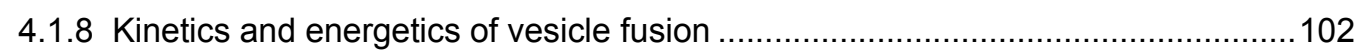

4.2 Minimal number of Synaptotagmin1 required to trigger fusion .............................. 104

4.3 A readily retrievable pool of synaptic vesicle constituents .................................105

4.3.1 A novel exogenous tracer for studying exo-endocytosis of endogenous vesicle

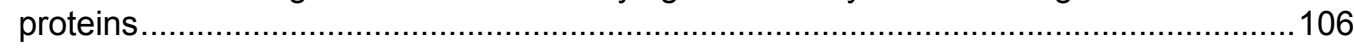

4.3.2 Stranded pool of vesicle proteins on the presynaptic membrane ......................106

4.3.3 Stimulation-dependent uptake of the readily retrievable pool........................108

4.3.4 Spatial organization of the readily retrievable pool ...................................110

4.3.5 Possible mechanisms of exo-endocytic coupling .......................................... 113

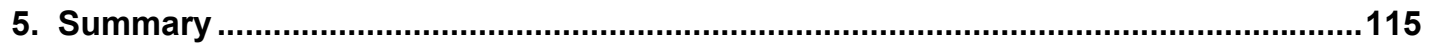

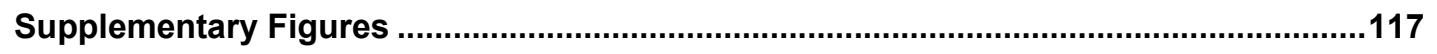

References ......................................................................................................... 119

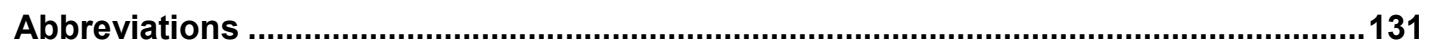

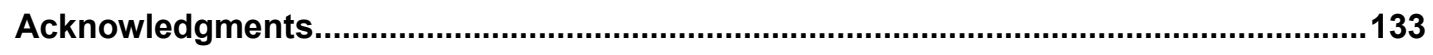

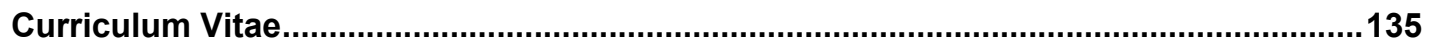

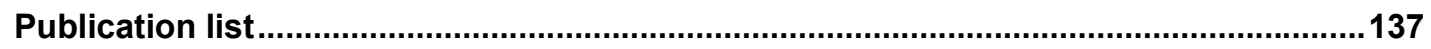




\section{TABLE OF FIGURES}

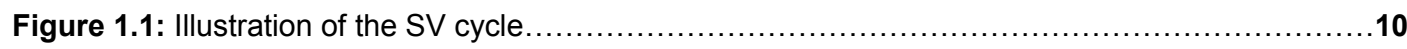

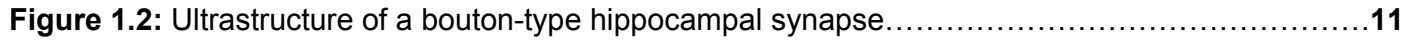

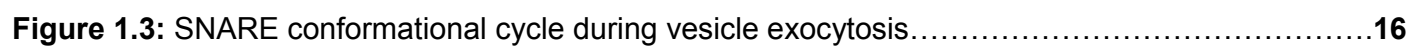

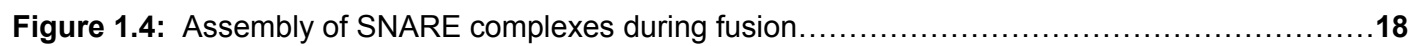

Figure 1.5: A multimeric model of SNARE-dependent membrane fusion ...........................21

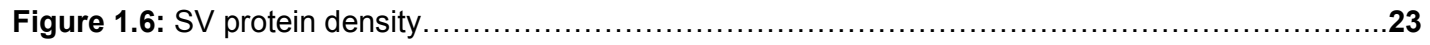

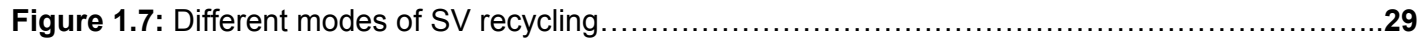

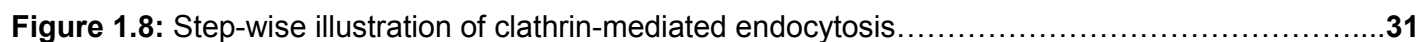

Figure 1.9: Three alternative modes of spatial and temporal coupling between exo- and endocytosis...34

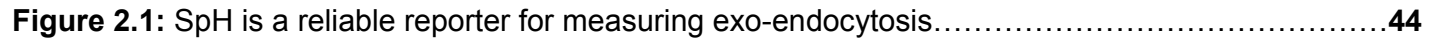

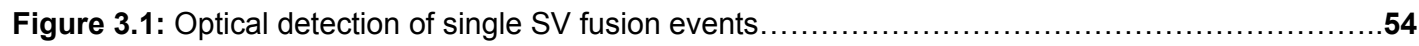

Figure 3.2: Prebleaching selectively attenuates the surface-stranded $\mathrm{SpH}$ without affecting the vesicular $\mathrm{SpH}$ fraction.

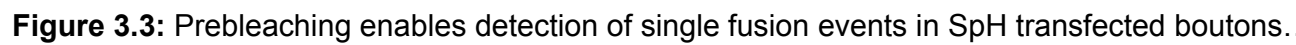
.56

Figure 3.4: $\Delta \mathrm{F}$ distributions of single AP fluorescence responses appear quantized. .58

Figure 3.5: In vitro single molecule imaging reveals only few copies of pHI-tagged proteins targeted to SVs.

Figure 3.6: Single molecule fluorescence intensity quantified from bleaching steps during neuronal measurements.

Figure 3.7: $\mathrm{SpH}$ overexpression in Syb2/Ceb DKO neurons rescues evoked synaptic transmission......66

Figure 3.8: Fluorescence responses to 40 and 100 APs are unaltered in SpH-overexpressing DKO boutons.

Figure 3.9: Two copies of $\mathrm{SpH}$ are minimally required to drive SV fusion.....

Figure 3.10: SVs with one copy of $\mathrm{SpH}$ are unable to fuse

Figure 3.11: Effect of missing one molecule peak in DKO histograms on net fluorescence amplitude...72

Figure 3.12: No cleavage of $\mathrm{pH}$ upon $\mathrm{SpH}$ overexpression in $\mathrm{DKO}$ neurons 
Figure 3.13: One Syt1-pHI molecule is targeted per SV when overexpressed on a WT background.....76

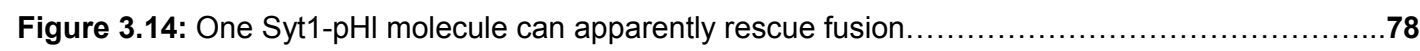

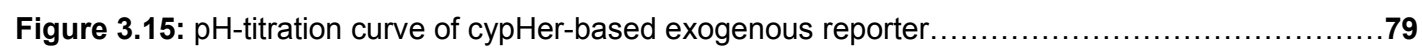

Figure 3.16: Live-cell labeling of hippocampal neurons with cypHer-coupled antibodies...............81

Figure 3.17: Fluorescence response of the cypHer probe upon exo-endocytosis.....................83

Figure 3.18: Dose-response curve to analyse the size of the surface pool...........................85

Figure 3.19: Comparison of SV recycling kinetics probed with $\mathrm{SpH}$ and cypHer-based technique.......87

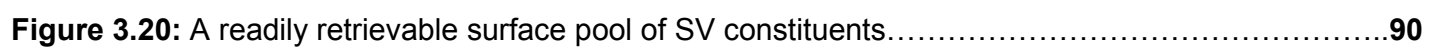

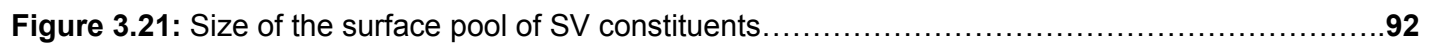

Figure 3.22: Sequential stimulation reveals reuse of the readily retrievable pool.......................93

Figure 4.1: An illustration of SV fusion driven by two SNARE complexes during neuroexocytosis.....101

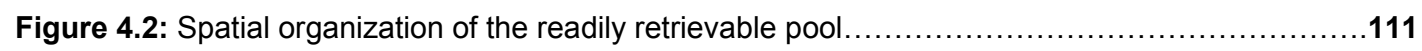

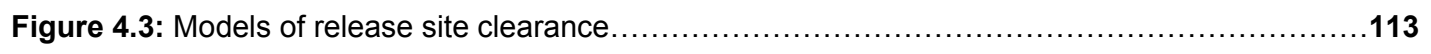

Figure S1: Comparison of standard deviations of $\Delta \mathrm{F}$ distributions from single molecule and single AP experiments. 117

Figure S2: An average of 2-3 copies of pHI-tagged proteins are targeted per SV. 118 


\section{ABSTRACT}

At conventional synapses of the central nervous system (CNS), fast synaptic transmission is mediated by the release of neurotransmitters (NTs) upon $\mathrm{Ca}^{2+}$-triggered synaptic vesicle (SV) exocytosis. Upon exocytosis SV proteins have to be resorted and retrieved from the surface by compensatory endocytosis in order to replenish the pool of NT-filled SVs. For my thesis I used $\mathrm{pH}$-switchable dyes, both genetically encoded as well as new exogenous ones, for optically analysing SV protein molecules necessary for fusion as well as their retrieval.

Exocytosis is mediated by the assembly of low-energy SNARE (soluble N-ethylmaleimidesensitive factor attachment protein receptor) protein complexes formed by the coil-coiling of three SNARE proteins: Synaptosomal associated protein - 25 (SNAP-25), Syntaxin 1A (Syx1A), and Synaptobrevin 2 (Syb2). However, it is unknown how many SNARE complexes are minimally needed for SV priming and fusion at CNS synapses. To resolve this issue, single vesicle fusion events were optically measured in real time using the genetically encoded probe SynaptopHluorin $(\mathrm{SpH})$, a $\mathrm{pH}$-sensitive green fluorescent protein (GFP), pHluorin $(\mathrm{pHI})$ fused to the luminal domain of the SV SNARE Syb2. Fluorescence responses upon fusion displayed a quantal distribution of $\mathrm{SpH}$ molecules into SVs. Quantitative single molecule experiments revealed that the quantal size corresponds to single $\mathrm{SpH}$ molecule fluorescence. Surprisingly, when overexpressed on a genetic null background, $\mathrm{SpH}$ could fully rescue evoked SV fusion. However, SVs expressing only one copy of SpH were unable to rapidly fuse upon stimulation. Taken together, the first part of the study demonstrates that two copies of $\mathrm{SpH}$ and hence two SNARE complexes are necessary and sufficient for SV fusion during fast synaptic transmission.

In order to maintain a steady-state rate of synaptic transmission the fused SV constituents are retrieved for further rounds of use by a compensatory process of endocytosis. Although clathrin-mediated endocytosis $(\mathrm{CME})$ is thought to be the predominant mechanism of SV recycling, it seems too slow to account for fast recycling. Therefore, it has been suggested that a pre-sorted and pre-assembled pool of SV proteins on the presynaptic membrane might support the first phase of CME. In the second part of this study the spatial and temporal 
dynamics of such a 'readily retrievable pool' (RRetP) of SV proteins at the presynaptic membrane of hippocampal neurons was monitored using a novel probe. By applying CypHer $5 \mathrm{E}$, a new cyanine dye-based $\mathrm{pH}$-sensitive exogenous marker, coupled to antibodies against luminal domains of SV proteins, the preferential retrieval of native SV constituents from the RRetP upon exocytosis was demonstrated. The functional size and capacity of this pool was found to closely resemble that of the readily releasable pool (RRP) of docked and primed SVs, suggesting that the RRetP can sustain SV recycling during moderate synaptic activity. Thus, the second part of the thesis demonstrates that small central synapses can avoid SV depletion in response to mild stimulation by having a preassembled pool of 'ready-to-go' SV constituents (RRetP), which efficiently supports compensatory endocytosis to a significant degree without relying on freshly exocytosed SV constituents. 


\section{INTRODUCTION}

Intercellular and intracellular signaling in eukaryotic cells is mediated by trafficking of membrane-delimited organelles (vesicles) between source and target membranes. Fusion of vesicles with the limiting membrane in a process called 'exocytosis', is a fundamental mechanism whereby cells discharge their chemical contents such as hormones, NTs etc. into the extracellular milieu and thereby mediate cell-cell signaling (for review see (Jahn, 2004; Jahn et al., 2003)). In the nervous system such signaling occurs between neurons at the level of specialized junctions called synapses. Arrival of an electric impulse (termed action potential $(A P))$ at the presynaptic terminal leads to $\mathrm{Ca}^{2+}$ influx via voltage-gated calcium channels, which then triggers exocytosis of fusion-competent SVs with the plasma membrane and hence discharge of their NT content into the cleft (Katz and Miledi, 1965, 1967). NT release is a well coordinated cyclic process (Fig. 1.1), whereby SVs are first loaded with NT, and then mobilized to the release site called the active zone (AZ). SVs initially dock and tether to the AZ and then undergo a series of maturation steps to gain fusion-competence (Fig. 1.1). $\mathrm{Ca}^{2+}$ influx upon a stimulus triggers exocytosis of SVs, which occurs within as little as 0.2 ms of an arrival of an AP (for review see (Lisman et al., 2007)). Such precise timing and high rates of $\mathrm{Ca}^{2+}$-triggered exocytosis implies that this process is tightly regulated both spatially and temporally. To achieve such precision and regulation this process is orchestrated by multiprotein complexes involved in a wide array of protein-protein and protein-lipid interactions (for review see (Rizo and Rosenmund, 2008; Sudhof, 2004)). Over three decades of intense research has led to a detailed characterization of the highly conserved protein family, SNAREs, that constitute the core fusion machinery, and other SNARE-associated components mediating exocytosis (for review see (Jahn and Scheller, 2006)). However, there are several fundamental mechanistic details that remain unresolved illustrating the complexity of this process. One such challenge is to understand the precise stochiometry of the fusion machinery necessary and sufficient for a single SV exocytosis and hence develop a minimalistic model of evoked membrane fusion. 


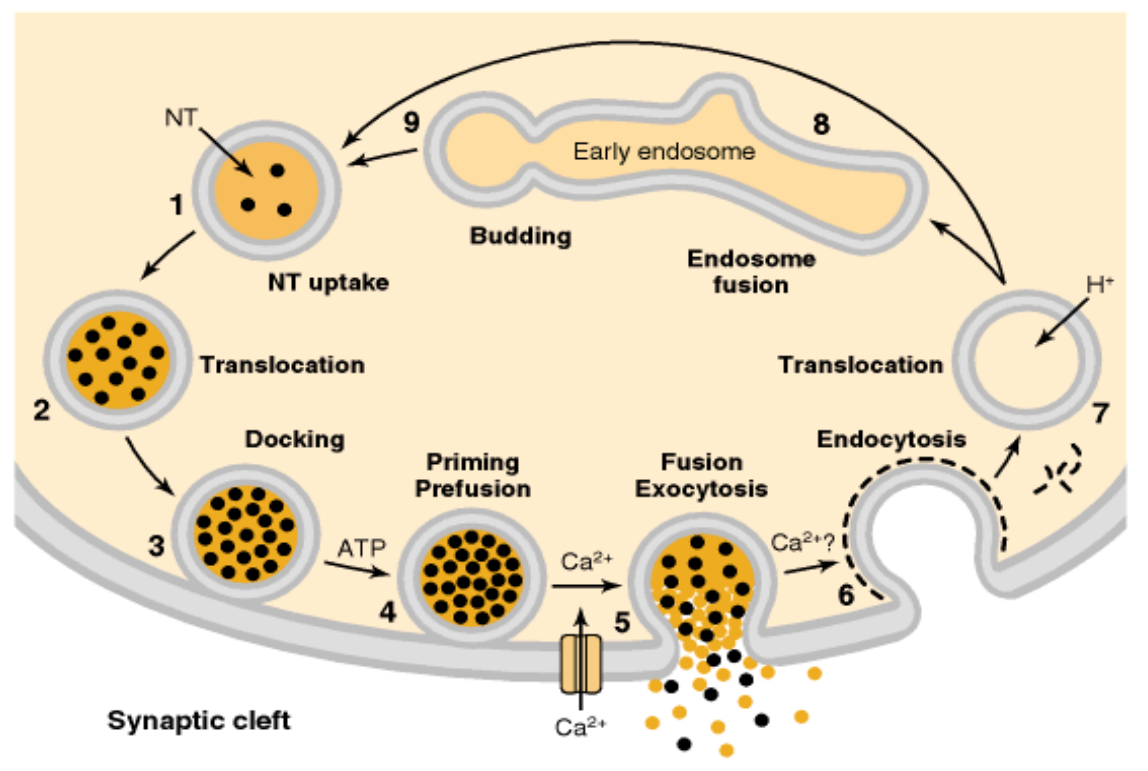

Figure 1.1: Illustration of the SV cycle.

The pathway of SVs in the nerve terminal is divided into several stages. Empty SVs take up NTs by active transport into their lumen (1). Packed SVs are transported to the AZ (2) where they undergo sequential steps of docking and tethering to the $A Z$ (3), priming for fusion (4) and exocytosis of vesicles upon $\mathrm{Ca}^{2+}$ influx through voltage-gated channels (5). Post-fusion SV constituents are retrieved by compensatory endocytosis (6). SVs reacidify via proton pump activity (7) and are then transported to early endosomes (8), intermediate sorting compartments followed by budding from endosomes (9). However, the endosomal processing can be circumvented and SVs can go directly from step 7 to step 1. Taken from (Sudhof, 1995).

To maintain a steady-state rate of synaptic transmission, SV constituents post-fusion are recycled from the plasma membrane by a compensatory process of endocytosis (Fig. 1.1) (Ceccarelli et al., 1973; Heuser and Reese, 1973). Consequently, when vesicle recycling by endocytosis is blocked, synapse activity soon declines (Koenig and Ikeda, 1989). Although several mechanisms of SV protein retrieval and recycling exist, the classical clathrin-mediated endocytosis (CME) represents the major pathway of SV recycling (Dittman and Ryan, 2009; Granseth et al., 2006). CME, similar to exocytosis, involves a network of complex intermolecular interactions but occurs on a much slower timescale and might be the ratelimiting step in SV recycling (for review see (Smith et al., 2008)). Despite its slow kinetics, compensatory endocytosis is able to support synaptic transmission during continuous stimulation at physiological frequencies (for review see (Schweizer and Ryan, 2006)). Thus, one of the central questions that continues to be intensely investigated is how the two 
processes of exocytosis and endocytosis are coupled spatially and temporally to sustain continuous synaptic transmission.

\subsection{Neuroexocytosis}

\subsubsection{Synaptic vesicle fusion}

SV fusion during neuroexocytosis is the fastest form of intracellular membrane fusion reaction (for review see (Rizo and Rosenmund, 2008)). Such high throughput rates and temporal precision, which enables a post-synaptic response within sub-milliseconds of presynaptic $\mathrm{Ca}^{2+}$ influx, is attributed to a well structured release process. Initially, filled SVs are transported to the presynaptic membrane where they attach at specialized sites for fusion on the membrane, active zones (AZs) (Couteaux and Pecot-Dechavassine, 1970; Landis, 1988). At the ultrastructural level the $A Z$ appears as an electron-dense structure associated with a dense cytomatrix (Fig. 1.2) (Harlow et al., 1998). It is composed of several multi-domain proteins, which interact with closely located voltage-gated calcium channels, other presynaptic and also SV proteins thereby ensuring rapid focal exocytosis and efficient stimulus-secretion coupling (for review see (Schoch and Gundelfinger, 2006)).

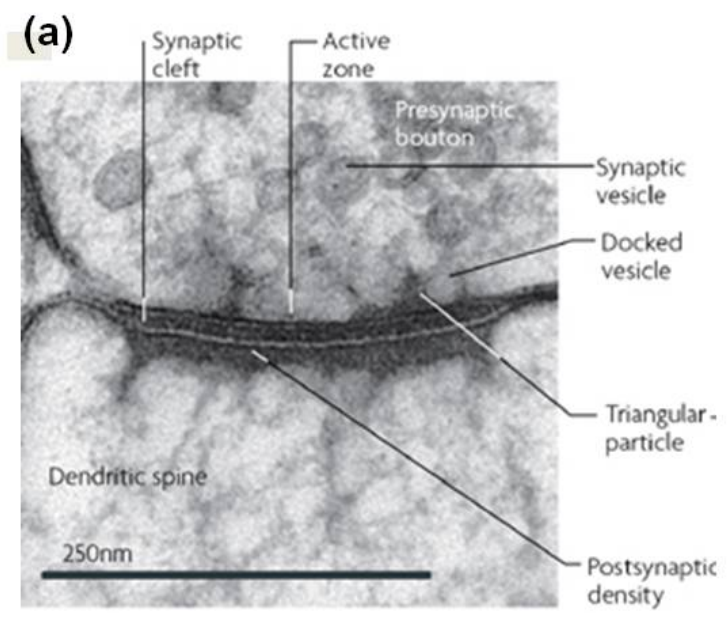

(b)

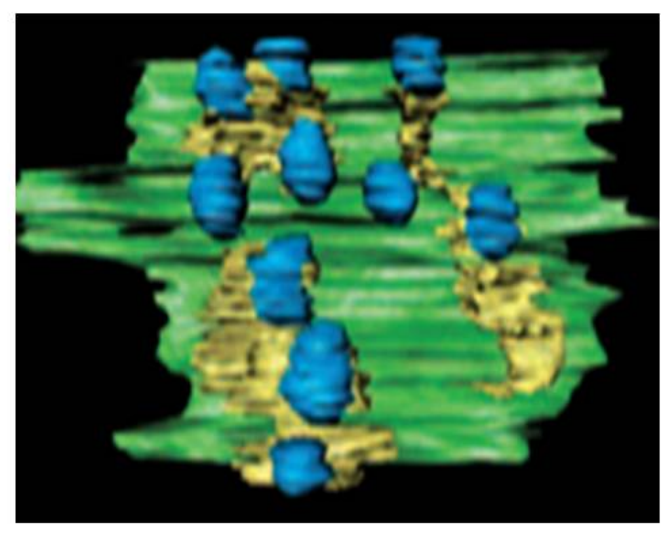

Figure 1.2: Ultrastructure of a bouton-type hippocampal synapse.

(a) Electron micrograph of a synapse in a hippocampal slice preparation made by high-pressure freezing, which avoids artifacts due to fixation. (b) An EM reconstruction of the AZ illustrating the dense AZ cytomatrix (yellow) and the docked SVs (blue). Taken from (Lisman et al., 2007). 
The initial physical attachment of a SV to the AZ prior to exocytosis is termed as docking (Steyer et al., 1997). Docking is more morphologically defined and the precise molecular underpinning is still unresolved (Fig. 1.1 and 1.2) (Verhage and Sorensen, 2008). Ultrastructural analysis based on proximity of SVs to the presynaptic membrane has been used to characterize docking, although the selection thresholds for such distance measurements vary among researchers (de Wit et al., 2006; de Wit et al., 2009; Hammarlund et al., 2007; Siksou et al., 2009). In central synapses there is a pool of SVs docked at the presynaptic membrane. However, their functional state can vary, which is why the RRP of SVs depleted upon a brief stimulus might differ in number to those docked at the membrane (Rettig and Neher, 2002; Rosenmund and Stevens, 1996). Therefore, docking of a SV is an intermediate maturation state, wherein the SVs are not yet release-ready (for review see (Verhage and Sorensen, 2008)). Docking is followed by a subsequent priming reaction whereby SVs gain fusion competence (Sorensen, 2004). Priming has been shown to be an ATP and $\mathrm{Ca}^{2+}$-dependent process, after which SVs are in a metastable state ready for fast $\mathrm{Ca}^{2+}$-triggered exocytosis (Chen et al., 2001; Eliasson et al., 1997; Kawasaki et al., 1998). It has been proposed that priming may occur by hemifusion (Reese et al., 2005; Xu et al., 2005), a process in which the proximal leaflets of the plasma- and vesicle membranes merge (allowing transfer of membrane markers), without fusion of the distal leaflets. Both docking and priming involve several molecular interactions between SV and presynaptic membrane proteins including SNAREs and several accessory cytosolic proteins. Previous studies have firmly implicated Munc-18-1 (member of the Sec1/Munc18-like protein family) and Syx1A (presynaptic membrane SNARE; part of the core fusion machinery) in docking (de Wit et al., 2006; Voets et al., 2001). Both proteins have been shown to interact with each other (Dulubova et al., 1999) and deletion mutants of either protein exhibit severe docking phenotypes (Hammarlund et al., 2007; Weimer et al., 2003). A recent study has identified the vesicle counterpart for docking as Synaptotagmin-1 (Syt1), the calcium sensor for exocytosis (de Wit et al., 2009). Additional factors such as rab3 and rab27, rabphilin3A, granuphilin, and exophilin4/SIp2a, have also been implicated in vesicle docking (for review see (Verhage and Sorensen, 2008)). Based on loss of function models several genes have been identified such 
as Munc-13 and calcium-activator protein for secretion (CAPS), which affected priming (Brose et al., 2000; Jockusch et al., 2007). Although it is difficult to clearly separate the docking and priming reactions, it is believed that the two processes are coupled whereby docking occurs upstream of priming.

\subsubsection{Calcium triggering of exocytosis}

Once SVs attain fusion competence, arrival of an AP leads to influx of $\mathrm{Ca}^{2+}$, which then triggers fusion. Entry of $\mathrm{Ca}^{2+}$ via $\mathrm{P} / \mathrm{Q}$-type or $\mathrm{N}$-type calcium channels leads to a rapid elevation in its concentration in the immediate vicinity called 'microdomain' typically about 200 $\mathrm{nm}$ in central synapses (Meinrenken et al., 2003). Previous studies have proposed that there are several $\mathrm{Ca}^{2+}$ channels clustered within this microdomain generating a gradient of $\mathrm{Ca}^{2+}$ concentration resulting in varying release probabilities $\left(\mathrm{P}_{\mathrm{r}}\right)$ among the docked and primed SVS (Meinrenken et al., 2002; Wadel et al., 2007). The RRP of docked and primed SVs containing on an average of 10 SVs (Harlow et al., 2001; Rosenmund et al., 2003; Schikorski and Stevens, 1997) have a very low $\mathrm{P}_{\mathrm{r}}$ at resting cytosolic $\mathrm{Ca}^{2+}$ concentration, giving rise to baseline spontaneous fusion called 'miniature post synaptic potentials' or 'minis' (Katz and Miledi, 1969; Lou et al., 2005). Upon stimulation the probability of fusion is enhanced due to $\mathrm{Ca}^{2+}$ elevation and the low background rate of spontaneous release (1-2 vesicle per min) (Geppert et al., 1994; Murthy and Stevens, 1999) switches to a fast synchronized form of neurosecretion.

Syt1 has been identified as the sensor for fast synchronous release at central synapses (Chapman, 2008; Koh and Bellen, 2003). Neurons lacking Syt1 display complete absence of rapid evoked release (Geppert et al., 1994). Moreover, the $\mathrm{Ca}^{2+}$ sensitivity of fusion is alleviated in response to a reduced $\mathrm{Ca}^{2+}$ binding affinity of Syt1, which further implicated its role in $\mathrm{Ca}^{2+}$ sensing (Fernandez-Chacon et al., 2001; Pang et al., 2006). About four to five $\mathrm{Ca}^{2+}$ ions bind co-operatively to and activate the calcium sensor Syt1, which thereby triggers vesicle release within $0.2-0.3 \mathrm{~ms}$ of $\mathrm{Ca}^{2+}$ elevation (Bollmann et al., 2000; Dodge and Rahamimoff, 1967; Schneggenburger and Neher, 2000). Thus, the calcium sensor Syt1 is a non-linear detector, which makes it very sensitive to alterations in $\mathrm{Ca}^{2+}$ concentration. Since 
only few Syt1 molecules may get activated, they are largely unsaturated during an AP and cause release of only a fraction of the RRP (for review see (Lisman et al., 2007). Syt1 is thus required for the fast and coordinated stimulus-secretion coupling during neurotransmission (Geppert et al., 1994). Closure of the calcium channels and diffusion causes the $\mathrm{Ca}^{2+}$ concentration to fall and prevents further activation of the sensor. Syt1 consists of distinct $\mathrm{Ca}^{2+}$-binding modules called the $\mathrm{C} 2$ domains (Chapman, 2008). The $\mathrm{C} 2$ domains also bind to phospholipids in a $\mathrm{Ca}^{2+}$-dependent manner and are thought to induce membrane curvature, which is essential for facilitating fusion (Groffen et al., 2010; Martens et al., 2008). Furthermore $\mathrm{Ca}^{2+}$-dependent interaction of $\mathrm{C} 2$ domains with SNARE proteins may stabilize some of the transient curved conformations during fusion (Martens et al., 2007).

\subsubsection{Minimal fusion machinery; SNAREs}

Membrane fusion is mediated and regulated by a wide array of membrane bound and soluble factors, which help in overcoming several energy barriers during this process. The evolutionarily conserved SNARE family of proteins constitutes the basic fusion machinery and mediate all intracellular fusion event in the secretory pathway (for review see (Jahn et al., 2003; Jahn and Scheller, 2006; Martens and McMahon, 2008)). The SNARE proteins are characterized by a conserved stretch of 60-70 amino acids called 'SNARE motifs' that are arranged in heptad repeats and have the tendency to form coiled-coils (for review see (Jahn and Scheller, 2006)). In neuronal synapses the core of the exocytosis machinery is formed by three members of the SNARE protein family: vesicle SNARE protein (v-SNARE), Syb2 and the presynaptic membrane SNAREs (t-SNARE) Syx1A and SNAP-25 (Jahn and Scheller, 2006; Sollner et al., 1993a; Sollner et al., 1993b). These SNAREs assemble into a highly stable heterotrimeric 'SNARE complex' that drives fusion. Syb2 and Syx1A contribute one SNARE motif each, whereas SNAP-25 contributes two SNARE motifs joined by a pamitoylated linker region. Most SNAREs contain a modular structure with a transmembrane domain (TMD) at the C-terminal end, which is coupled to the SNARE motif by a short linker (Kloepper et al., 2007). The central role of these three synaptic SNAREs in fusion was demonstrated when they were found as specific substrates of clostridial neurotoxins (tetanus and botulinum toxins), which via 
their catalytic zinc-endopeptidase domain specifically cleave SNAREs resulting in inhibition of NT release and subsequent paralysis (Schiavo et al., 2000; Simpson, 2004). Besides, targeted deletion of each of the synaptic SNAREs leads to an almost complete impairment of neuroexocytosis (Schoch et al., 2001; Sorensen et al., 2003).

During NT release the SNARE proteins undergo a series of conformational changes, which mediates interaction between the SV and the presynaptic membrane resulting in fusion (Fig. 1.3). The SNAREs assemble into a core complex with equimolar stoichometry $(1: 1: 1)$ by coilcoiling of the four $\alpha$-helical SNARE motifs. On the basis of the highly conserved central layer of the four helix bundle in the core complex (three glutamines $(Q)$, one arginine $(R)$ ), the participating SNAREs have been categorized into Qa- SNAREs (Syx1A), Qb- (SNAP-25) Qc(SNAP-25) and R-SNAREs (Syb2) (Bock et al., 2001; Fasshauer et al., 1998b; Sutton et al., 1998). One copy of each $Q$ and R-SNAREs contribute to the formation of a functional SNARE complex (Fig.1.3). 


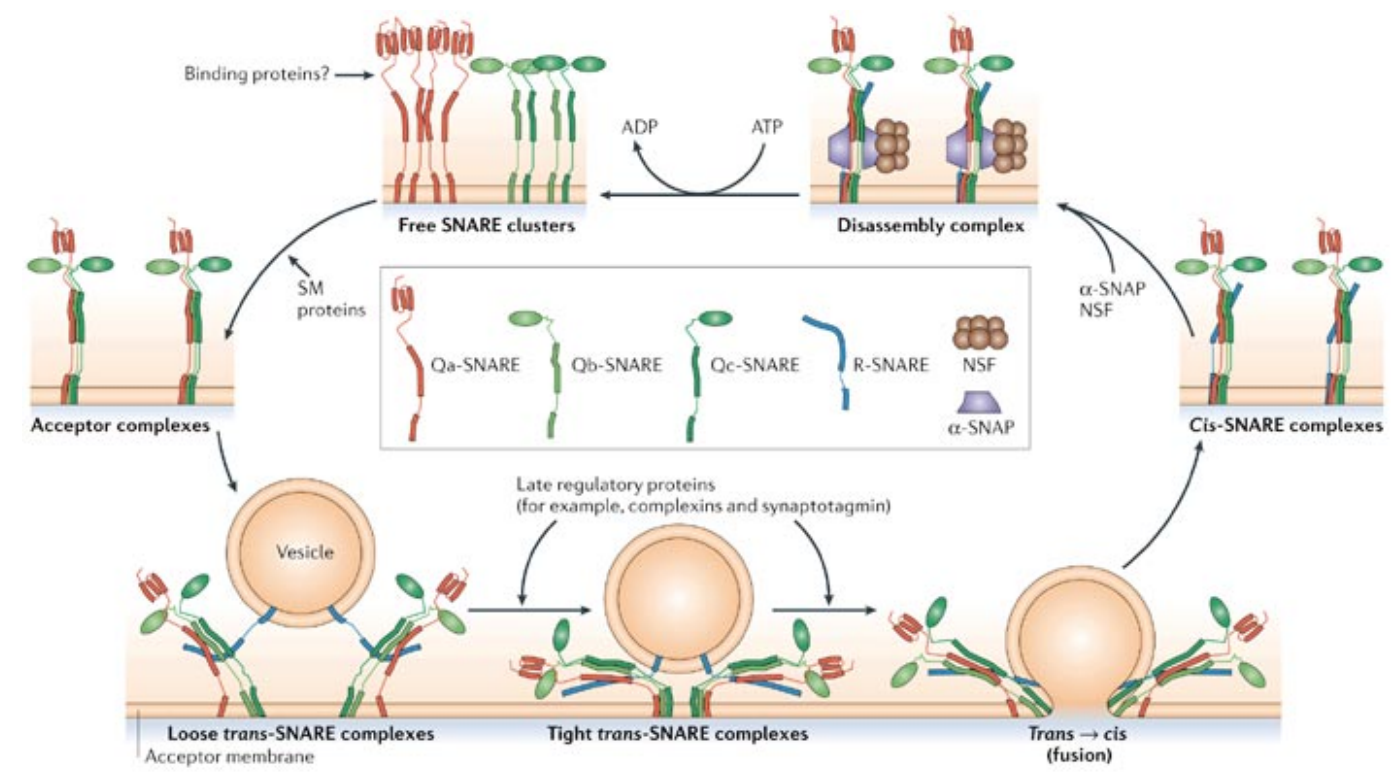

Figure 1.3: SNARE conformational cycle during vesicle exocytosis.

Initially the Q-SNAREs (Syx1A and SNAP-25) on the acceptor membrane exist as free clusters which assemble into acceptor complexes mediated by the SM (Sec1/Munc18-related) proteins. These acceptor complexes then interact with vesicular R-SNARE, Syb2, to form a loose trans-SNARE complex initiated by nucleation of the complex on the N-terminal region of the SNARE motif of the R-SNARE. This is followed by the 'zippering' of SNARE motifs from their N-terminal portion to the C-terminal region, which leads to a tight complex causing membrane merger and formation of a transient fusion pore, through which vesicular contents are released. Other proteins like, complexin and Syt regulate the different transition states during exocytosis. The tight trans-complex relaxes into the cis-complex during fusion which is later disassembled by the concerted action of NSF (N-ethylmaleimide-sensitive factor) and $\alpha$ SNAP (soluble NSF attachment protein). Taken from (Jahn and Scheller, 2006).

The SNARE proteins in the presynaptic membrane associate to form stable acceptor complexes before the start of fusion (Fasshauer and Margittai, 2004; Fiebig et al., 1999). The fusion reaction is initiated when the acceptor complexes of Q-SNAREs in the target membrane interact with the N-terminal region of the SNARE motif of R-SNARE, Syb2, leading to the formation of a loose SNARE complex in a trans-configuration, where the two interacting TMDs are located in the two opposing bilayers (Fig. 1.3). Zipper-like assembly of the SNARE motifs from their $\mathrm{N}$-termini towards their membrane-proximal $\mathrm{C}$-termini results in the formation of a highly stable heterotrimeric tight trans-SNARE complex, consisting of four parallel alpha helices (Fig. 1.3) (Hanson et al., 1997; Hu et al., 2003; Lin and Scheller, 1997; Weber et al., 
1998). Trans-complex synthesis is thought to be reversible and they shuttle in between the loose and tight states maintaining a dynamic equilibrium (Fig 1.4) (for review see (Jahn and Scheller, 2006)). The trans-SNARE complex, also referred to as the SNAREpin due to its similarity to the hairpin bundle formed during viral fusion (Fig. 1.4) (Weber et al., 1998), pulls the two membranes into close proximity and triggers fusion. It is believed that the energy liberated during the formation of the four helix bundle and the mechanical force generated due to the strain on the linker regions, acting as force transducers, is sufficient to overcome the activation energy barrier for phospholipid merger (Fig. 1.4) (Li et al., 2007). Indeed when the synaptic SNAREs were reconstituted into separate populations of synthetic liposomes, they were able to drive membrane fusion which led to the idea that SNAREs are the 'minimal fusion machinery' (Weber et al., 1998). However, these in vitro assays have, thus far, not been able to reproduce the time-course and the spatial control of neuroexocytosis as in vivo, which could be attributed to the absence of numerous accessory proteins involved in the tight regulation of this process. Although SNARE complex formation is the key element for fusion, there are several auxiliary factors such as Munc 18-1, Munc13 and rab3 inertacting molecule (RIM), Syt1 and complexins that interact with and stabilize this complex, but the precise nature and sequence of these interactions remain unclear (for review see (Rizo and Rosenmund, 2008)). Therefore it is rather possible that the basic apparatus for regulated fusion could extend beyond the SNAREs.

As the phospholipid bilayers of the two opposing membranes merge, the trans-SNARE complex relaxes into a cis-complex, which is later disassembled by the concerted action of the ATPase $\mathrm{N}$-ethylmaleimide sensitive factor (NSF) and its adaptor protein soluble NSF attachment protein ( $\alpha$-SNAP), thus regenerating free SNARE molecules for subsequent rounds of fusion (Sollner et al., 1993a). 
(a)

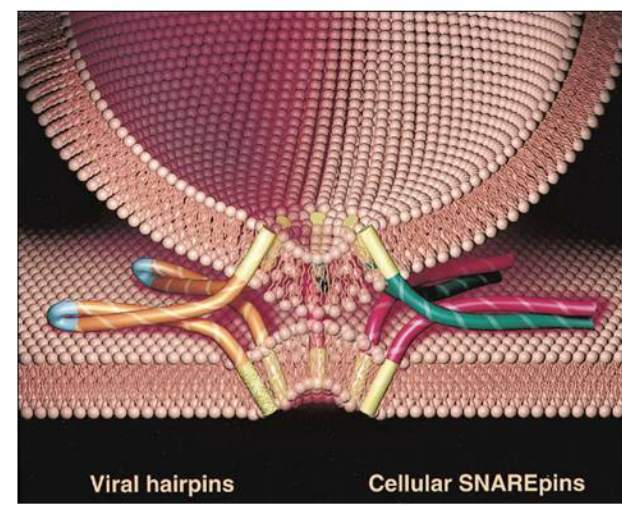

(b)

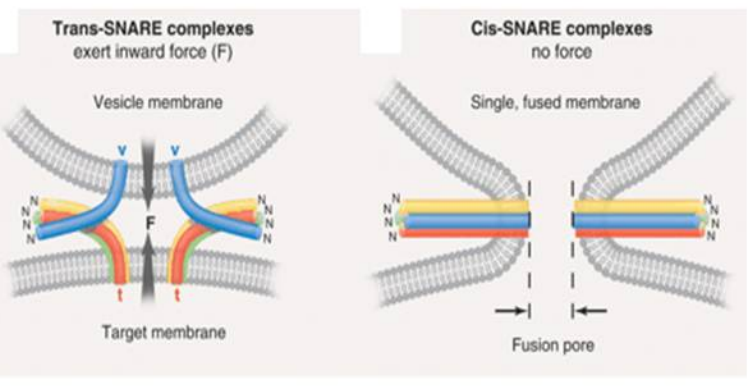

Figure 1.4: Assembly of SNARE complexes during fusion.

(a) Trans-SNARE complexes called SNAREpins formed between cognate v-SNAREs (green) and plasma membrane t-SNAREs (red). The SNAREpin configuration closely resembles the viral hairpin structure formed by a single fusion protein anchored at both the donor and target membrane. Taken from (Weber et al., 1998). (b) Zipper-like assembly of SNAREs during SV fusion. Progressive assembly from the $\mathrm{N}$-terminus towards the $\mathrm{C}$-terminus pulls the two membranes close together exerting an inward force and triggering fusion. Taken from (Sudhof and Rothman, 2009).

\subsubsection{Fusion pore}

During exocytosis the two opposing membranes merge and lead to the formation of a fluidfilled connection called the fusion pore bridging the two aqueous compartments. The fusion pore is a transient intermediate structure of a few nanometers capable of conducting ions and NTs. Indeed patch-clamp amperometric recordings, that enable measurement of NT release from neuroendocrine cells, clearly show an initial 'foot-signal' characteristic of restricted release via fusion pore followed by a spike indicating full release (Alvarez de Toledo et al., 1993). Other techniques, such as cell-attached capacitance measurements, have also been employed to study fusion pore conductance (He et al., 2006; Lindau and Alvarez de Toledo, 2003). These recordings have revealed that the fusion pore is a metastable state and can either close after a transient connection with the plasma membrane as observed in several examples of 'stand-alone foot signals' in amperometric measurements or could evolve into full collapse fusion which would result in a typical amperometric spike (Alvarez de Toledo et al., 1993; He et al., 2006; Jackson and Chapman, 2008; Klyachko and Jackson, 2002; Neher and 
Marty, 1982). It has been proposed that the membrane bending action of the C2 domains in Syt1 molecule creates significant curvature stress which might aid in the opening of the fusion pore and subsequent expansion (Martens et al., 2007). There has been a long-standing debate about the molecular nature of the fusion pore. As a result, there are contrasting models where some propose that the fusion pore is a proteinaceous gap-junction like channel that extends across both the bilayers, while others postulate that the pore is entirely lipidic and hence less rigid (Jackson and Chapman, 2008; Lindau and Almers, 1995). Results in the present study provide some insight into this controversy.

\subsubsection{Minimal number of SNARE complexes necessary for fusion}

One of the most debated topics of membrane fusion that still remains unresolved is the minimum number of SNARE complexes thermodynamically and kinetically sufficient to drive a single vesicle fusion event during rapid neurotransmission? Although single SNARE proteins can assemble into the four-helix bundle of SNAREpin, they have been shown to form higherorder multimeric complexes (Antonin et al., 2000; Fasshauer et al., 1998a; Hayashi et al., 1994; Poirier et al., 1998; Tokumaru et al., 2001). Such a multimeric model for membrane fusion would be consistent with viral fusion where a minimum cooperativity of three hemagglutinin trimers for influenza virus and five trimers for Semliki Forest virus has been observed (Danieli et al., 1996; Gibbons et al., 2004). It was postulated that such a multimeric super-complex could be formed if the two SNARE domains, contributed by SNAP-25, participate in separate SNARE complexes and hence cross-link this multimeric structure (Fig. 1.5) (Tokumaru et al., 2001; Weber et al., 1998). This led to the proposal that several of these SNARE complexes might assemble in a rosette-shaped multivalent super-complex, forming a ring, around the fusion pore (Fig. 1.5); however, there is no direct evidence in support of this model (Montecucco et al., 2005; Tokumaru et al., 2001; Weber et al., 1998). Furthermore, it is difficult to envisage the accumulation of multiple SNARE complexes into the small fusion pore without affecting membrane integrity. From an energetic point of view it was recently shown that the stabilization energy of a single SNARE complex in vitro, which is $\sim 70 \%$ assembled, is very high (35 $\left.\mathrm{k}_{\mathrm{B}} \mathrm{T}\right)$ (Li et al., 2007). Considering the activation energy for lipid bilayer fusion (50 
- $100 k_{B} T$ ), it was suggested that only a few of these complexes could be sufficient to drive fusion (for review see (Rizo and Rosenmund, 2008)). Thus, the precise number of SNARE complexes minimally required to drive membrane fusion is highly debated and current estimates range between 1 and 15 (Bowen et al., 2004; Domanska et al., 2009; Han et al., 2004; Hua and Scheller, 2001; Karatekin et al.; Keller et al., 2004; Li et al., 2007; Mohrmann et al., 2010; van den Bogaart et al., 2010). The disparities in these results arise largely because of the indirect nature of the determinations and the different model systems used. Some of these results are described below.

In an initial study to estimate the number of SNARE complexes sufficient for fusion, the inhibition of dense core vesicle fusion in cracked PC12 cells caused by a soluble SNARE domain was monitored as a function of its concentration (Hua and Scheller, 2001). The doseresponse curve of inhibition of NT release by a truncated Syb2, due to the formation of a nonfusogenic SNARE complex, is thus an indicator of the cooperativity of SNARE proteins. Based on theoretical fitting of the experimental data to a model, it was estimated that three SNARE complexes cooperate to mediate fusion of a single dense core vesicle. Surprisingly, this number was quite similar to the $\mathrm{Ca}^{2+}$ cooperativity for NT release $(n=4)$. Thus, it was suggested that there could be a one-to-one coupling between the calcium sensor, Syt1, and the SNARE complexes (Stewart et al., 2000). Thus, it would be also interesting to quantify the minimum number of Syt1 molecules required to initiate fusion. In a later study, cell-attached capacitance recordings were performed on PC12 cells to measure the effect of Syx1A TMD mutations on fusion pore conductance (Han et al., 2004). Based on a steric hindrance model it was proposed that the fusion pore is lined by five to eight copies of Syx1A TMDs during exocytosis of a single granule, thereby almost doubling the previous estimate. A much higher number $(10-15)$ was suggested for SV exocytosis from dose-response curves, where NT release was inhibited with botulinum neurotoxin serotype A (BoNT/A) (Keller et al., 2004). BoNT/A specifically cleaves SNAP-25 but the product is still able to engage in stable SNARE complex formation with Syb2 and Syx1A thereby leading to a long-term effect. Assuming that SNAP-25 bridges multiple SNARE complexes involved in fusion, the ratio of the half maximal inhibitory concentration $\left(\mathrm{IC}_{50}\right)$ for SNAP-25 cleavage to that of the NT release was suggested 
to yield the stochiometry of the multimeric SNARE super-complex. However, the major drawback of this study was the assumption that every cleaved SNAP-25 molecule must participate in SNARE pairing (Montecucco et al., 2005). Based on the above studies a multimeric model of SV fusion emerged where an array of SNARE complexes arranged in a ring around the fusion pore was thought to be essential for rapid-regulated secretion (Fig. 1.5).

(a)

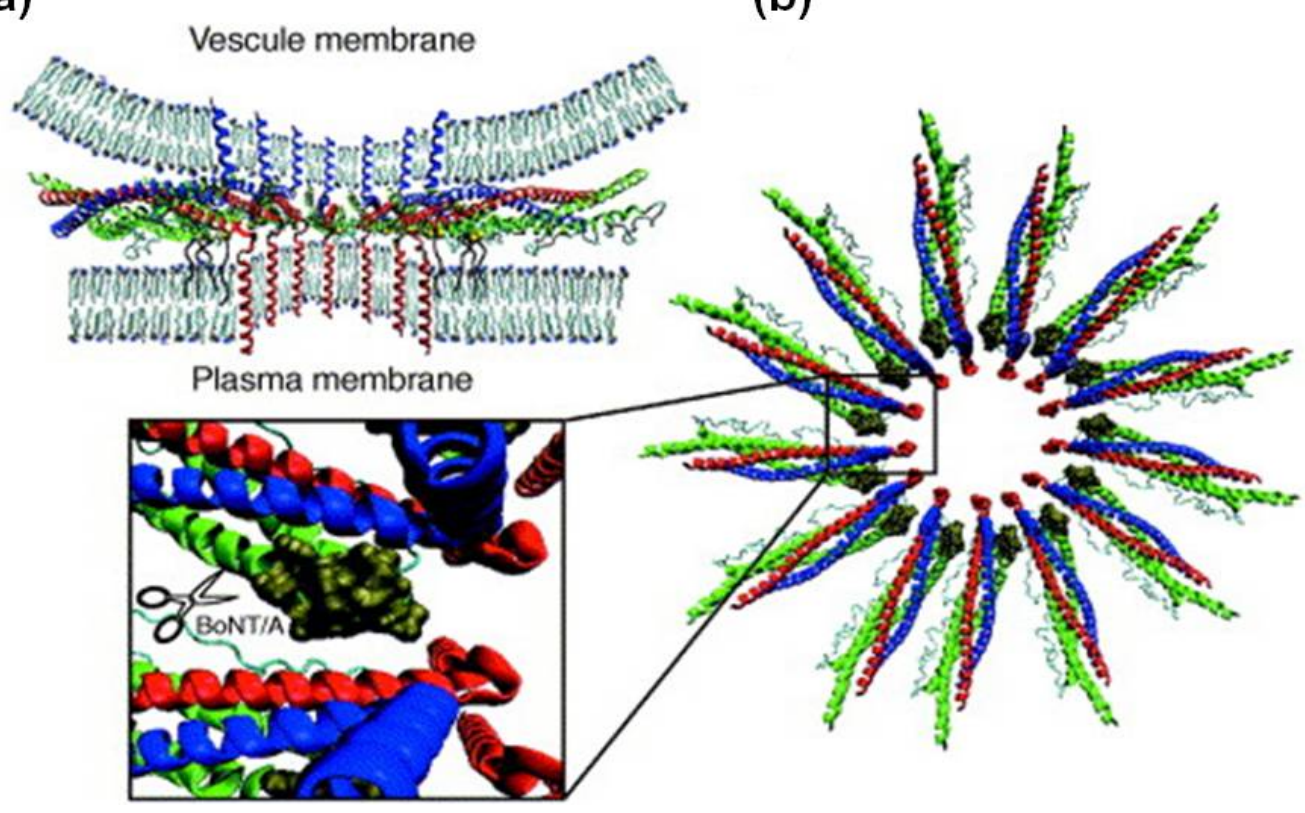

Figure 1.5: A multimeric model of SNARE-dependent membrane fusion.

(a) Side view of the cross-section of the multivalent SNARE supercomplex arranged in a circular array around the fusion pore.

(b) Top view of the dodecamer of SNARE complexes.

Taken from (Montecucco et al., 2005).

Recently, in vitro Förster resonance energy transfer (FRET) experiments have been used to measure SNARE-mediated fusion at very low protein-to-lipid $(\mathrm{p} / \mathrm{l})$ ratios (van den Bogaart et al., 2010). The results from these experiments contradict the requirement of a SNAREsupercomplex for SV fusion. Using liposomes constituted with an average of one SNARE 
protein, they could clearly demonstrate that there is no cooperativity in SNAREpin formation and only one SNARE complex is sufficient for fusion with either liposomes containing complementary SNAREs or even with purified SVs.

The latest attempt to resolve the number of SNARE complexes necessary for fusion was made in live chromaffin cells by titrating the ratio of wild-type and mutant SNARE proteins expressed (Mohrmann et al., 2010). The observations indicated a third-power relation for fast, synchronous fusion indicating that exocytosis of dense-core granules require at least three functional SNARE complexes quite consistent with a previous estimate in PC12 cells (Hua and Scheller, 2001; Mohrmann et al., 2010).

Thus, the number of estimated SNARE complexes for vesicle fusion is quite heterogenous and could depend on various factors, such as kinetics of the fusion reaction and size of the fusing organelle. Moreover, most of the above reports are inferential and indirect extrapolations based on theoretical models and more importantly do not address this issue in an intact neuronal and physiological setting. Therefore, it is essential to apply a more direct method capable of visualizing single SNARE molecules during fast $\mathrm{Ca}^{2+}$-triggered exocytosis and hence in a neuronal setting.

\subsubsection{Copy numbers of synaptic vesicle proteins}

A detailed quantitative picture of the molecular composition of a rat SV was generated in 2006 by Takamori et al using purified SVs from rat brain (Takamori et al., 2006). The copy numbers of the major SV proteins was determined and a structural model was built based on their abundance. The SV proteome analysis revealed most of the previously described membrane proteins along with a few novel ones (Takamori et al., 2006). The SV membrane was found to be packed with integral membrane proteins with a quarter of the entire surface volume occupied by TMDs. On quantifying the average copy numbers of the major proteins per SV, a typical SV was found to contain on an average 70 Syb2 molecules, 32 Synaptophysin (Syp) molecules, 9-14 NT transporters, 15 Syt1 molecules and only 1 copy of the proton pump, vATPase (Fig. 1.6). It is noteworthy that there is such huge variability in densities among 
different proteins such as between the V-ATPase, which is crucial for NT filling of SVs and Syp, which seems to be functionally dispensable. Although there could be heterogeneity in the numbers between individual SVs, it is striking to find such a high surface density of R-SNARE Syb2 on the SV. Since the number of SNARE complexes required, although widely debated, is far less, it was postulated that by having an excess repertoire of the SV fusogen, it would provide a safe reserve pool of SNAREs for continuous exo-endocytosis. This would mean that Syb2 density is not rate-limiting for fusion. A recent study estimated absolute protein numbers on purified SV using a single molecule approach and found on average relatively low levels of Syb2 (Mutch et al., 2011).

(a)

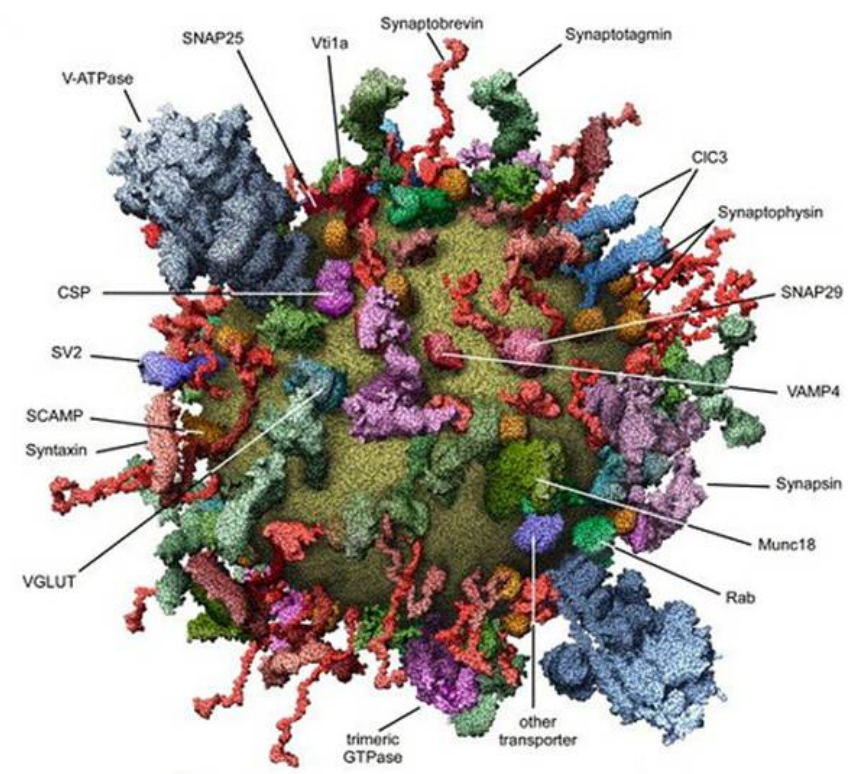

(b)

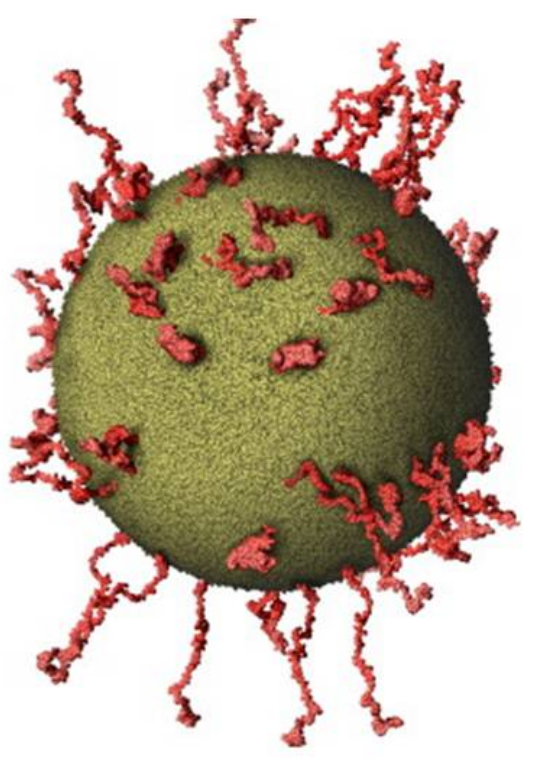

Figure 1.6: SV protein density.

(a) A space-filling model illustrating the surface density of a rat SV.

(b) Overview of the relative abundance of only Syb2 on a SV. Average copy number of Syb2 per SV is 70.

Taken from (Takamori et al., 2006). 
Densities of $Q$ or t-SNAREs on the plasma membrane were recently measured during single granule exocytosis in live PC12 cells using total internal reflection fluorescence microscopy (TIRFM) (Knowles et al., 2010). Fluorescent clusters of Syx1a and SNAP-25 were observed on the plasma membrane, where single granules had docked, and it was estimated that about 50-70 copies each of Syx1a and SNAP-25 molecules in each cluster, although only a small fraction of these might actually engage in SNARE complex formation. Since the size of the secretory granules in PC12 cells is much larger than SV (Bowser and Khakh, 2007) and therefore might differ in SNARE density, it was interesting to see that the t-SNARE copy numbers closely matched that of 70 Syb2 per SV as estimated by Takamori et al (Takamori et al., 2006). Thus, several attempts have been made to estimate SNARE densities on vesicle and target membrane, but it has not been possible to visualize the effective number of SNAREs mediating fusion in live cells. Moreover, relatively little is known about the effect of alterations in SNARE density on SV exocytosis during fast synaptic transmission.

\subsubsection{Visualizing SNARE molecules during single vesicle fusion}

Single molecule fluorescence assays monitoring docking and fusion of SNARE reconstituted liposomes with supported bilayers reconstituted with complementary SNAREs, were able to resolve single vesicle release and estimate the number of participating SNARE proteins. An initial attempt based on such a single molecule approach, found that as low as one SNARE complex is sufficient for docking and fusion (Bowen et al., 2004). Docked liposomes were incubated with BoNT/B such that all the uncomplexed Syb2 were cleaved and only the fraction incorporated in SNARE complex remained unperturbed. However, a recent study on single liposome fusion kinetics reported a number of six to nine SNARE complexes based on models of sigmoidal cumulative distribution of delay times before fusion, which were attributed to multiple activation steps of SNARE complex folding (Domanska et al., 2009). Another study based on similar experiments reported dramatic reduction in fusion rates when Syb2 number per liposomes was lowered and hence suggested similar numbers of five to ten SNAREpins necessary for fusion (Karatekin et al., 2010). Although the fusion reactions in this study were 
significantly fast ( $20 \mathrm{~ms})$ over previous in vitro assays (Pobbati et al., 2006), they did not mimic the physiology and biochemistry of triggered exocytosis in an intact synaptic setting.

Therefore, as a first step to address the minimum SNARE density on a SV, required to trigger fusion, it is essential to resolve single SV fusion in an intact physiological setting. With the advent of pH-sensitive GFPs, pHluorins (pHI), it is possible to label v-SNAREs, Syb2, by coupling $\mathrm{pHI}$ to its lumenal domain $(\mathrm{SpH})($ Miesenbock et al., 1998) and then count the number of $\mathrm{SpH}$ incorporated per SV. Due to their $\mathrm{pH}$-dependent fluorescence property, these genetic reporters can monitor dynamic changes in $\mathrm{pH}$ of $\mathrm{SV}$ lumen resulting from exocytosis and endocytosis of SVs during presynaptic activity (Sankaranarayanan et al., 2000). Subsequently, other SV proteins such as Syt1, Syp and vesicle glutamate transporter (vGlut) have also been conjugated to $\mathrm{pHI}$ to optically track their dynamics during SV recycling (Balaji and Ryan, 2007; Granseth et al., 2006; Sankaranarayanan and Ryan, 2000). Due to the optimal signal-to-noise ratio of these probes, it has been possible to resolve fusion of even single SVs in hippocampal synapses in response to a single stimulus (Balaji and Ryan, 2007; Gandhi and Stevens, 2003; Zhu et al., 2009). Based on the low coefficient of variation in fluorescence amplitude distributions of single SV fusion and further GFP fluorescence calibrations, it was suggested that only a few copies of $\mathrm{SpH}$ get incorporated on a per SV basis (Balaji and Ryan, 2007; Fernandez-Alfonso and Ryan, 2004; Gandhi and Stevens, 2003). Since these measurements were performed against the background of the endogenous complement of 70 Syb2 copies, it was difficult to assess the actual number of Syb2 necessary for fusion. Thus, it has not been feasible to count the minimum number of SNAREs required during single vesicle fusion in vivo.

\subsubsection{Single molecule optical methods to determine protein copy numbers}

Single molecule fluorescence assays have enabled visualization of individual fluorophores even in live cells (Joo et al., 2008; Vale, 2008). Thus, such single molecule analysis can provide a direct assay to count protein copy numbers in cells by overexpressing fluorescent fusion chimeras with the protein of interest. At low density, isolated fluorophores appear as diffraction-limited spots. Since a fluorophore has a limited number of excitation/emission 
cycles, after certain duration it gets irreversibly photobleached. Such bleaching events appear as fast instantaneous fluorescence downsteps and are diagnostic of single molecules (Pierce et al., 1997; Ulbrich and Isacoff, 2007; Vale et al., 1996). Multiple bleaching steps indicate the presence of several fluorophores in the diffraction-limited spot. Studies based on such singlemolecule bleaching analysis, have precisely counted the number of subunits of membranebound proteins in live cells, such as postsynaptic N-methyl D-aspartate (NMDA) receptors, voltage-activated phosphatase Ci-VSP, proton channel Hv1 and the voltage-gated potassium channel KCNQ1 (Kohout et al., 2008; Nakajo et al., 2010; Tombola et al., 2008; Ulbrich and Isacoff, 2007). Other studies based on similar single molecule photobleaching measurements have determined the subunit composition of the calcium channel complex, cAMP-regulated chloride channel and bacterial flagellar proteins (Ji et al., 2008; Leake et al., 2006).

Since the amplitude of single molecule bleaching steps is a direct correlate of single molecule fluorescence, once calibrated the fluorescence intensity can be used to estimate the protein content in terms of absolute numbers in a complex or in a particular region of the cell. This method has been used to determine the absolute number of post-synaptic protein molecules in single hippocampal synapses or number of protein subunits that bind to microtubules (Gestaut et al., 2008; Sugiyama et al., 2005). The above single molecule measurements are largely performed with high-resolution microscopy such as TIRFM, which selectively illuminates and excites fluorophores in a restricted region of the specimen immediately adjacent to the optical interface along the z-axis, hence eliminating the background fluorescence and considerably improving the axial resolution. Thus, it has been used extensively to monitor single fluorescent molecules or processes that occur at or close to the plasma membrane (for review see (Axelrod, 2003)). However, recent advances in detectors with single photon sensitivity allow single molecule detection even with epifluorescence microscopy.

The present study has applied similar single-molecule fluorescence microscopy with genetically encoded probes, in hippocampal botuons, to label individual SNARE molecules 
and then determine the precise stoichiometry of SNARE complexes sufficient to execute membrane fusion during fast synaptic transmission. 


\subsection{Synaptic vesicle recycling; different forms of endocytosis}

The presynaptic terminal of small CNS synapses draw upon a limited pool of recycling SVs and the rate at which this pool is replenished determines the maximal steady-state rate of neurotransmission. Retrieval of fused SVs from the presynaptic membrane by endocytosis for further rounds of use is a crucial and rate-limiting step for replenishment and thus maintaining continuous release without complete depletion of the recycling pool (for review see (Smith et al., 2008)). Several modes of SV retrieval have been proposed but the major pathway is considered to be the classical CME (Fig. 1.7) (Brodin et al., 2000; Brodsky et al., 2001; Cremona and De Camilli, 1997; Heuser and Reese, 1973). In this classical pathway there is full-collapse fusion of the SV membrane with the plasmalemma followed by clathrin-mediated invagination and fission during endocytosis (Heuser and Reese, 1973; Pearse, 1976; Wu et al., 2007). This process is relatively slow and is thought to occur at a timescale of tens of seconds to minutes (Mueller et al., 2004; Voglmaier and Edwards, 2007; Wu et al., 2007). In order to sustain fast synaptic transmission, however, it was suggested that an alternative rapid 'kiss and run' mechanism of SV turnover might occur with a time constant of $1-2 \mathrm{~s}$, whereby the vesicle transiently fuses with the membrane and reseals before full collapse hence retaining its molecular identity (Fig. 1.7) (Aravanis et al., 2003; Ceccarelli et al., 1973; Gandhi and Stevens, 2003; He et al., 2006; Koenig et al., 1998; Zhang et al., 2009). During elevated neuronal activity a third pathway called bulk endocytosis is recruited wherein large pieces of membrane are retrieved directly from the plasma membrane in a clathrin-independent manner (Fig. 1.7) (Holt et al., 2003; Koenig and Ikeda, 1989, 1996; Richards et al., 2000; Takei et al., 1996; Teng and Wilkinson, 2000; Wu and Wu, 2007). 


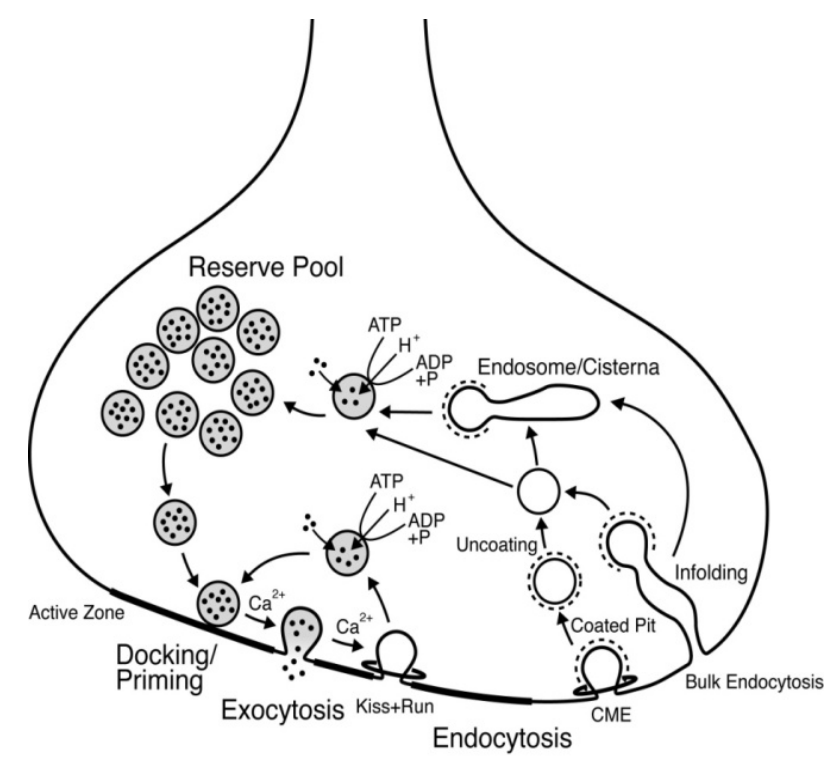

Figure 1.7: Different modes of SV recycling.

(courtesy J. Klingauf)

\subsubsection{Clathrin-mediated endocytosis}

The clathrin-dependent mode of recycling was popularized based on the classical ultrastructural studies of Heuser and Reese (Heuser and Reese, 1973), where they induced a tetanic stimulation of the frog neuromuscular junction, and observed a subseqeunt decrease in SV number together with an increase in the number of coated vesicles and cisternae, which gradually reversed with formation of new SVs. Based on these observations, they proposed that following full-collapse fusion of SVs, there is retrieval of SV constituents by coated pits which recycle through endosomal intermediates. This model has been widely supported in several preparations including CNS synapses using optical assays based on fluorescent dyes, GFP-tagged SV proteins and intraluminal antibodies (for review see (Rizzoli and Jahn, 2007)). One such assay based on pHI-tagged SV proteins (Miesenbock et al., 1998), has been successfully used to monitor exo-endocytosis even at the level of single SVs (Balaji and Ryan, 2007; Gandhi and Stevens, 2003; Granseth et al., 2006; Sankaranarayanan et al., 2000). Using these probes in hippocampal synapses it has been demonstrated that there is a single 
mode of endocytosis with a time-constant of $\sim 14-15 \mathrm{~s}$, and fast endocytosis is just at one end of this distribution (Balaji and Ryan, 2007; Granseth et al., 2006).

The molecular basis of clathrin-mediated endocytosis is not well understood at small CNS synapses, although a lot is known about the process from other cell-types. It a stepwise process, which involves a cascade of interaction between a wide array of proteins, that form an extensive network with the plasma membrane and the underlying actin cytoskeleton (Fig. 1.8) (for review see (Takei and Haucke, 2001)). First, there is recruitment of the adaptor protein, AP-2, Stonin 2 (also known as stoned B) (Diril et al., 2006) and perhaps AP180, which sort specific SV constituents and recruit individual clathrin subunits called 'triskelia' at the cytosolic surface of the plasma membrane forming a clathrin-coated pit at a site away from the AZ (Fig. 1.8) (for review see (Perrais and Merrifield, 2005; Ryan, 2006)). Next, there is polymerization of clathrin heavy and light chain at the pit to form a hexagonal lattice around the invaginating vesicle (Fig. 1.8) (Cremona and De Camilli, 1997; Gundelfinger et al., 2003). During the final stages of endocytosis, the clathrin-coated vesicle is pinched off at its neck by the co-ordinated action of the GTPase dynamin and perhaps a transient burst of actin polymerization (Fig. 1.8) (Cremona and De Camilli, 1997; Merrifield et al., 2002; Sankaranarayanan et al., 2003). The clathrin coat is then rapidly shed in an ATP-dependent reaction carried out by the polyphosphoinositide phosphatase, synaptojanin and the molecular chaperones Hsc70 and Auxilin (Fig. 1.8) (Cremona et al., 1999; Eisenberg and Greene, 2007; Harris et al., 2000; Verstreken et al., 2003). During CME there is progressive recruitment of a range of SH3 domain proteins such as Intersectin, Ampiphysin, Endophilin, Syndapin and other factors which mediate extensive protein-protein and protein-lipid interactions for a productive synergy between the plasma membrane, endocytic machinery and the actin cytoskeleton (Haucke et al., 2011; Ryan, 2006; Slepnev and De Camilli, 2000). Acute perturbation by knockdown or by gene deletion of the key components, such as clathrin and dynamin, in hippocampal synapses result in near-complete inhibition of stimulation-dependent endocytosis (Ferguson et al., 2007; Granseth et al., 2006; Heerssen et al., 2008). This illustrates that most of the SV endocytosis is dependent on the classical clathrin-coated pathway. 


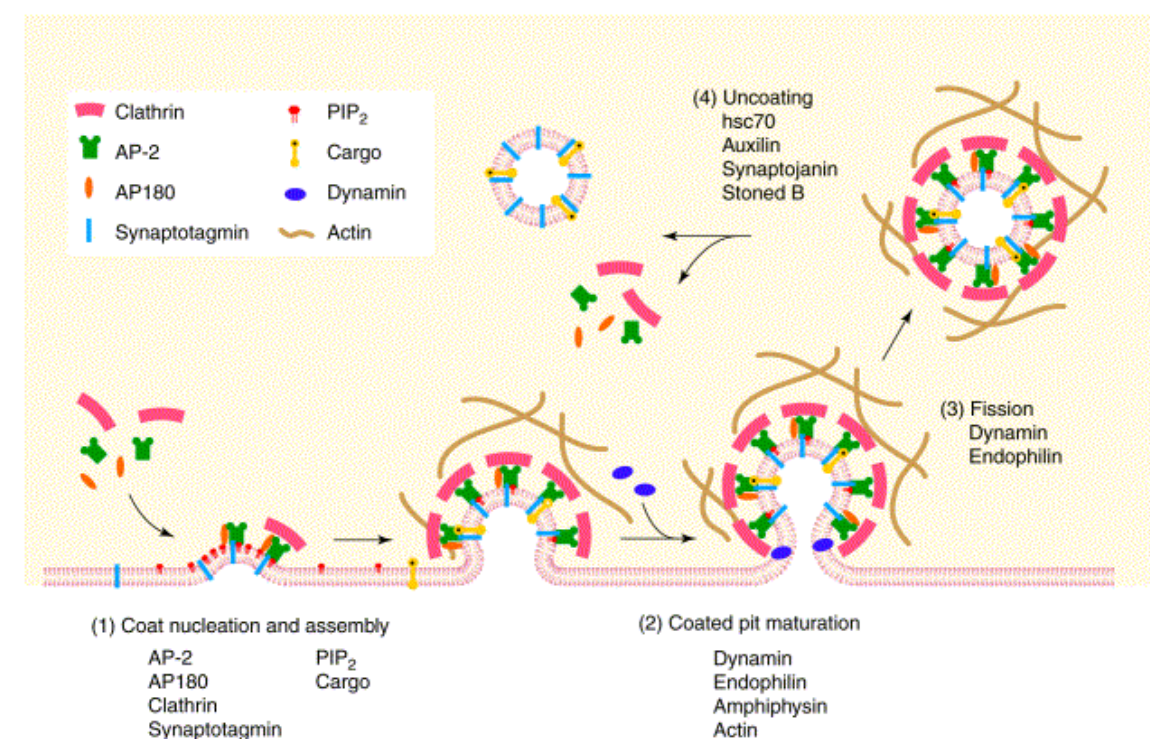

Figure 1.8: Step-wise illustration of clathrin-mediated endocytosis.

Taken from (Takei and Haucke, 2001).

\subsubsection{Kiss-and-run mode of recycling}

Classical studies by Ceccarelli and his co-workers had led to the proposal that SV fusion occurs transiently via the opening of a small fusion pore followed by rapid closure without full collapse of the SV membrane (Ceccarelli et al., 1972, 1973). This model, whereby the SV retains its biochemical identity was later called 'kiss-and-run' largely due to EM micrographs of frog NMJs, after a low frequency stimulation, which exhibited an increase in SVs that acquired the tracer and no considerable increase in coated vesicles. Furthermore, there was an increased incidence of uncoated 'omega' membrane profiles with a narrow neck attached to the plasma membrane at the AZ (Ceccarelli et al., 1973; He and Wu, 2007; Koenig et al., 1998), however it was unclear whether these SVs are going to fully fuse or will reseal their neck. Due to the apparent kinetic advantage of this pathway, it was suggested that this could be a rapid and efficient form of endocytosis, which might prevent SV pool depletion during periods of high activity. Moreover, this mode of SV fusion allows the synapse to restrict the release of transmitters through the narrow fusion pore resulting in a subquantal response (He et al., 2006; Klyachko and Jackson, 2002) compared to full collapse fusion, which might also 
be a mechanism to regulate synaptic strength (Choi et al., 2000). Although kiss-and-run mode of vesicle turnover exists in neuroendocrine cells, its presence at nerve terminals particularly CNS synapses has remained controversial. The strongest evidence in favour of this pathway was derived from optical assays based on partial destaining of single SV loaded with slowly dissociating ( 3 s) styryl FM dye FM1-43 (Aravanis et al., 2003; Klingauf et al., 1998; Pyle et al., 2000). The results indicated partial dye retention in SVs akin a kiss-and-run mode of fusion that was further confirmed using a hydrophilic FM1-43 quencher, bromophenol blue (Harata et al., 2006). In another optical study, Gandhi and Stevens imaged single SV exo-endocytosis by overexpressing $\mathrm{SpH}$ in hippocampal neurons. A rapid fluorescent transient with a retrieval time course of less than 1s was observed, which was ascribed to the fast kiss-and-run mode of retrieval (Gandhi and Stevens, 2003). Recent studies based on small pH-sensitive phospholuminescent 'quantum dots', have shown that SVs loaded with these tiny inorganic particles exhibited distinct patterns of photoluminescence upon kiss-and-run versus full collapse fusion (Zhang et al., 2007; Zhang et al., 2009). However, the relative contribution of the kiss-and-run pathway to exo-endocytosis at CNS synapses is activity dependent and remains highly debated.

\subsubsection{Bulk endocytosis}

In the original tracer experiments by Heuser and Reese at frog NMJs, large endosomes were observed, after moderate to heavy stimulation, which bud off clathrin coated SVs. This mode of recycling occurring via endosomes underlies the slower endocytosis rates (Danglot and Galli, 2007) and has been proposed to occur at motor nerve terminals of frog (Miller and Heuser, 1984; Richards et al., 2000) and snake (Teng and Wilkinson, 2000), endocrine cells (Neher and Marty, 1982; Rosenboom and Lindau, 1994; Thomas et al., 1994) and even at central synapses (de Lange et al., 2003; Gad et al., 1998; Holt et al., 2003; Paillart et al., 2003). After tetanic stimulation deep infoldings of the plasma membrane in continuity with the extracellular space are often observed which results in the retrieval of huge chunks of membrane by bulk endocytosis (Takei et al., 1996). Support for this model at CNS synapses has been further substantiated by recent EM tomography of dynamin-1 deletion mutants, 
which exhibit accumulation of deeply invaginated clathrin-coated pits connected to the plasma membrane upon heavy stimulation (Ferguson et al., 2007). Taken together, these studies suggest that this high-capacity pathway of bulk endocytosis is activity-dependent and is only recruited when the balance between exo- and endocytosis breaks down.

\subsubsection{Coupling of exo- and endocytosis}

During continuous synaptic activity and high rates of exocytosis, the reliability and kinetics of coupling between exo- and endocytosis becomes the rate-limiting step for SV recycling. Due to the complex molecular composition of a SV, which contains $\sim 200$ protein molecules (Sudhof, 2004; Takamori et al., 2006), it becomes a time-consuming process to recapture and resort the different vesicle membrane proteins in the right stoichiometry post-fusion to generate a fully functional SV. However, rapid kinetic modes of recycling like kiss-and-run can circumvent this problem by reversible fusion pore openings, which preserve the biochemical identity of the SV.

There are three possible modes of spatial and temporal coupling of exo- and endocytosis at small central synapses (Fig. 1.9). In the first scenario, SVs undergo kiss-and-run mode of vesicle turnover, where the vesicle undergoes a simple reversal of exocytosis and is retrieved intact without losing its molecular identity thereby spatially and temporally coupling exo- and endocytosis (Fig. 1.9). Such tight coupling between exo- and endocytosis has been demonstrated at the level of single SV (Aravanis et al., 2003; Gandhi and Stevens, 2003). In the second scenario, SVs undergo full collapse fusion but the vesicle constituents post-fusion remain clustered as a unit in raft-like patches (Fig. 1.9) (Martin, 2000; Thiele et al., 2000), diffusing along the presynaptic membrane to designated sites of retrieval (endocytic zone) where they get internalized thereby also preserving the molecular identity and coupling exoendocytosis (for review see (Rizzoli and Jahn, 2007)). In the third scenario, post-fusion SV constituents disperse and mix at the plasma membrane thereby losing its molecular identity and subsequent endocytosis preferentially retrieves from a pool of preassembled SV material at the endocytic site, hence uncoupling exo- and endocytosis (Fig. 1.9) (Fernandez-Alfonso et al., 2006; Wienisch and Klingauf, 2006). A study on single vesicle imaging in hippocampal 
neurons suggested an alternative mechanism, where it was shown that at low frequency stimulation, endocytosis occurs in two distinct kinetic steps; a fast mode ( 3 s) which preserves the identity of SVs exo- and endocytosed with high fidelity and a slow mode which retrieves the SV constituents from the surface (Zhu et al., 2009). This way, synapses can ensure both fast turnover and partial maintenance of identity during repeated rounds of exoendocytosis.

(a)
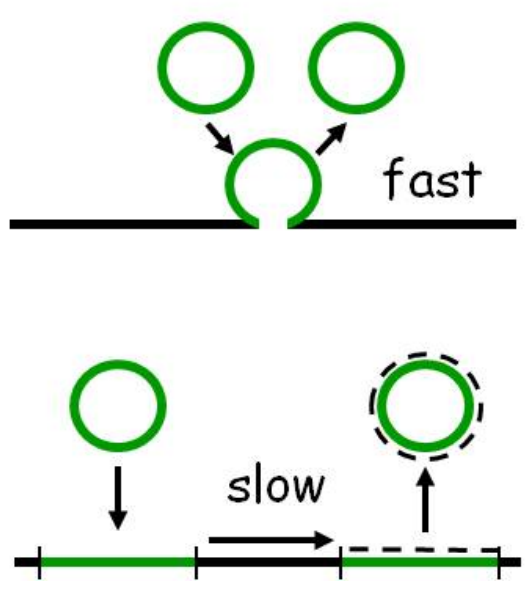

(b)

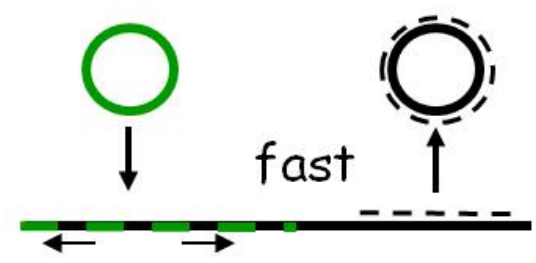

Figure 1.9: Three alternative modes of spatial and temporal coupling between exo- and endocytosis.

(a) Maintenance of biochemical identity of SVs post-fusion either due to a diffusion barrier like the fusion pore (kiss-and-run; upper left) or adherence of SV components to each other remaining as a unit (lipid rafts; lower left).

(b) Loss of molecular identity upon fusion due to dilution with the surface reservoir of SV constituents, which are preferentially retrieved during compensatory endocytosis.

Courtesy: M Wienisch and J Klingauf. 


\subsubsection{Readily retrievable pool of vesicle components}

It was proposed that in central synapses, SV constituents post-fusion mix and dilute with a surface pool of SV constituents, which are predominantly recruited upon subsequent endocytosis (Fernandez-Alfonso et al., 2006; Wienisch and Klingauf, 2006). Using a GFP fusion protein with the clathrin light chain, it was found that during the first $10 \mathrm{~s}$ of prolonged stimulation clathrin is not being recruited from the cytosol to form coated pits, although the rate of endocytosis measured with FM dyes is high (Mueller et al., 2004). These results suggested that SVs during the first phase are either retrieved by a clathrin-independent mechanism (kissand-run) or by preassembled 'ready-to-go' coat structures at regions near the AZ. Moreover, BoNT/A, which completely inhibits exocytosis, did not block endocytosis of a limited pool of SVs clearly suggesting that compensatory endocytosis draws upon pre-existing SV constituents at the presynaptic membrane (Neale et al., 1999).

Further support for a functional pool of preassembled structures at the presynaptic membrane came from experiments using fusion constructs of the SV proteins Syb2 and Syt1 with pHI (Miesenbock et al., 1998). These studies have shown that SVs post fusion lose their protein complement, and the molecular identity of SVs exocytosed and subsequently endocytosed is not conserved (Dittman and Kaplan, 2006; Fernandez-Alfonso et al., 2006; Wienisch and Klingauf, 2006). Based on these observations it was suggested that exocytosis and subsequent endocytosis is uncoupled and there may be a pool of preassembled SV proteins on the presynaptic membrane, which is preferentially retrieved upon exocytosis (Mueller et al., 2004; Wienisch and Klingauf, 2006). Previous studies using activity dependent markers in snake neuromuscular terminals have shown their accumulation at bouton margins upon stimulation which might represent endocytic zones (Teng et al., 1999; Teng and Wilkinson, 2000). This is in agreement with other ultrastructural and high-resolution microscopy analyses, which describe the presence of several SV proteins on the presynaptic membrane of resting synapses (Taubenblatt et al., 1999; Willig et al., 2006). Presence of such a surface complement of vesicle constituents has been postulated to represent a pre-clustered pool being immediately available for a first wave of endocytosis upon stimulation (Miller and 
Heuser, 1984; Wienisch and Klingauf, 2006). Transient overexpression of the genetic exoendocytic probe SpH (Miesenbock et al., 1998), in hippocampal boutons leads to targeting of up to $\sim 30 \%$ of the fusion protein to the bouton membrane, constituting a surface pool which participates in SV protein recycling during compensatory endocytosis (Fernandez-Alfonso et al., 2006; Wienisch and Klingauf, 2006).

The finding, however, that $\mathrm{pH}$ tagged versions of other SV proteins result in only minor surface expression, about $8 \%$ for Syp-pHI (Granseth et al., 2006) and even only about $2 \%$ for the glutamate transporter vGlut1-pHI (Balaji and Ryan, 2007) has called into question the existence of a surface pool of endogenous SV proteins. It has thus been suggested that the high surface expression of SpH and Syt1-pHI (Fernandez-Alfonso et al., 2006; Wienisch and Klingauf, 2006) might be a mere overexpression artefact, thereby discrediting the use of $\mathrm{pHI}$ as optical probes for exo-endocytosis (Opazo et al., 2010; Willig et al., 2006). Thus, endogenous SV proteins might be only transiently exposed to the surface during exoendocytosis and might even remain clustered thereby tightly coupling exo- and endocytosis (Willig et al., 2006), contrary to $\mathrm{pHI}$ based finding that SVs post fusion lose their identity during recycling (Wienisch and Klingauf, 2006). Thus, it is crucial to visualize the spatial and temporal kinetics of endogenous SV protein recycling and to directly visualize the retrieval from the "readily retrievable surface pool of synaptic vesicles" (RRetP), if existent. 


\subsection{Aim of the study}

The aim of my thesis was to explore the usability of $\mathrm{pH}$-switchable reporters, both genetically encoded as well as new exogenous ones, for studying trafficking of SV proteins during the exo-endocytic itinerary, if possible down to the single molecule level. Using these probes, I addressed two unresolved questions concerning SV exo-endocytosis:

1. How many SNARE complexes are necessary and sufficient for vesicle fusion during fast synaptic transmission?

To resolve the current disparity in estimates (1 to 15$)$ of the precise number of SNARE complexes required for membrane fusion, this study employed a direct approach to optically resolve single SNARE molecules mediating fusion and then count the minimum number of these molecules required to drive evoked fusion.

To achieve this, a four-step strategy was adopted:

a) Overexpress a fluorophore-tagged version of Syb2, like $\mathrm{SpH}$, which selectively switches on upon fusion thereby giving a specific read-out for exocytosis.

b) Optically resolve fusion of single SVs upon stimulation in hippocampal boutons.

c) Single molecule calibration to estimate the number of $\mathrm{SpH}$ molecules incorporated per SV.

d) Overexpress $\mathrm{SpH}$ on a genetically null background to determine the precise number of $\mathrm{SpH}$ molecules and hence the number of SNARE complexes minimally required to rescue evoked fusion.

Since the fusion apparatus works in close concert with the $\mathrm{Ca}^{2+}$-sensing machinery, it would be interesting to understand the molecular basis of the $\mathrm{Ca}^{2+}$-co-operativity for NT release. In simple terms, what is the minimum number of calcium sensors (Syt1) required for synchronicity of synaptic transmission? By overexpressing Syt1-pHI on a null background, the number of Syt1-pHI molecules required for single SV fusion was estimated. 
2. Is there a small functional surface pool of presorted and reclustered SV constituents constituting a 'readily retrievable pool' (RRetP) from which SVs are regenerated during compensatory endocytosis?

Previous studies have proposed the existence of a reservoir of SV components on the presynaptic membrane, which are preferentially endocytosed leading to molecular non-identity between exo- and subsequently endocytosed SVs. However, it was so far not possible to monitor the kinetics of this surface pool in real time. Therefore, to address this issue, I together with my colleague Yunfeng Hua took a new approach to label and monitor the spatio-temporal dynamics of endogenous SV proteins on the presynaptic membrane:

a) First, a novel pH-sensitive tracer, cypHer5E, coupled to antibodies against the luminal domains of Syt1 (aSyt1-cypHer) and the vesicular GABA transporter (VGAT; aVGAT-cypHer), was used to label the native SV proteins.

b) Live-cell imaging was performed to test the efficiency of these new exogenous probes for monitoring exo-endocytosis in real time.

c) Finally in combination with genetically encoded $\mathrm{pH}$-sensitive probes such as $\mathrm{SpH}$, the spatial and temporal kinetics of the endogenous surface pool of vesicle proteins was studied. 


\subsection{Primary hippocampal neuron culture}

Primary cultures of hippocampal neurons were prepared from the CA3/CA1 region of 1 or 2 day-old Wistar rats as previously described (Wienisch and Klingauf, 2006). The Synaptobrevin 2/ Cellubrevin (Syb2/Ceb) double knock-out (DKO) mice were obtained from Prof. Dieter Bruns (University of Saarland, Homburg, Germany). Since the Syb2/Ceb DKO mice are postnatally lethal (Borisovska et al., 2005; Schoch et al., 2001), hippocampi from embryonic day 18 (E18) mice were used. As wild-type (WT) control we used E18 pups from separately bred C57BL/6 mice. The hippocampi were dissected in ice-cold Hanks solution (Sigma) supplemented with $20 \%$ Fetal Bovine Serum (FBS) and cut into small sections. This was followed by enzymatic digestion for $10 \mathrm{~min}$ at $37^{\circ} \mathrm{C}$ with $2 \mathrm{ml}$ digestion solution containing 10 $\mathrm{mg}$ trypsin (Sigma) and $1 \mathrm{mg}$ DNase (Sigma). The tissue was then triturated with siliconized pasteur pipettes in $2 \mathrm{ml}$ dissociation solution containing $1 \mathrm{mg}$ DNase followed by centrifugation at $2000 \mathrm{rpm}$ for $5 \mathrm{~min}$ at $4^{\circ} \mathrm{C}$ to obtain a cell pellet. The cell pellet was resuspended in $\sim 2 \mathrm{ml}$ of plating medium and plated onto $18 \mathrm{~mm}$ coverslips which were coated with Matrigel (BecktonDickinson; diluted 1:50 in Hank's solution $+10 \%$ FBS). The cells were first incubated with plating medium containing $2 \mathrm{mM} \mathrm{L-glutamine} \mathrm{to} \mathrm{promote} \mathrm{glial} \mathrm{proliferation.} \mathrm{After} \mathrm{one} \mathrm{day} \mathrm{cells}$ were fed with growth medium containing either $2 \mu \mathrm{M}$ or $4 \mu \mathrm{M}$ of the mitosis blocker, cytosine arabinoside (Sigma), depending on the glial density. Cultures were maintained at $37^{\circ} \mathrm{C}$ in a 95 $\%$ air/ $5 \% \mathrm{CO}_{2}$ humidified incubator. Cultures were used after 14-16 days in vitro (DIV) for imaging experiments. All prenatal E18 neurons were grown on an astrocyte feeder layer at low density to promote long-term viability for the imaging experiments.

Solutions used for cell culture preparation:

Basic Medium: Minimal essential medium (MEM without phenol red, Gibco) with the following supplements per $500 \mathrm{ml}: 2.5 \mathrm{~g}$ glucose, $100 \mathrm{mg} \mathrm{NaHCO}$, $50 \mathrm{mg}$ Transferrin (Calbiochem).

Plating Medium: $100 \mathrm{ml}$ Medium contained $10 \%$ FBS, heat inactivated at $57^{\circ} \mathrm{C}$ for $30 \mathrm{~min}, 1$ $\mathrm{ml} 0.2 \mathrm{M}$ L-glutamine solution, $2.5 \mathrm{mg}$ insulin. 
Growth Medium: $100 \mathrm{ml}$ Medium contained $5 \%$ FBS, heat inactivated at $57{ }^{\circ} \mathrm{C}$ for $30 \mathrm{~min}$, $0.25 \mathrm{ml} \mathrm{0.2} \mathrm{M} \mathrm{L-glutamine} \mathrm{solution,} 2 \mathrm{ml}$ B-27-Supplement (Gibco), $4 \mu \mathrm{M}$ or $2 \mu \mathrm{M}$ cytosine arabinoside (Sigma).

Hank's Solution: Hank's balanced salt solution without calcium or magnesium (Sigma) plus 50 mg/l NaHCO3, 1 mM HEPES, pH adjusted to 7.3-7.4 with $\mathrm{NaOH}$

Hank's Solution with $20 \%$ FBS

Dissociation Solution: Hank's solution plus $12 \mathrm{mM} \mathrm{MgSO} 4 \cdot 7 \mathrm{H}_{2} \mathrm{O}$

Digestion Solution: $137 \mathrm{mM} \mathrm{NaCl}, 5 \mathrm{mM} \mathrm{KCl}, 7 \mathrm{mM} \mathrm{Na} \mathrm{HPO}_{4}, 25 \mathrm{mM}$ HEPES, adjusted with $\mathrm{NaOH}$ to $\mathrm{pH} 7.3-7.4$

FBS: Fetal bovine serum (Biochrom KG) Trypsin: type XI (Sigma)

DNase: type IV (Sigma)

Matrigel (Beckton-Dickinson): diluted 1:50 in Medium (MEM)

\subsection{Transfection}

Cultured neurons were transfected at 3 DIV by a modified calcium phosphate transfection procedure (Threadgill et al., 1997; Wienisch and Klingauf, 2006). First the growth medium was replaced with serum-free Neurobasal A Medium (NBA, Invitrogen) 30 min prior to transfection. The old growth medium was saved. A calcium phosphate/DNA precipitate was then formed by mixing 5-30 $\mu \mathrm{g} / \mathrm{ml}$ plasmid DNA, diluted in sterile double distilled water to a final concentration of $0.2 \mathrm{mg} / \mathrm{ml}$, with $5 \mu \mathrm{l}$ of $2.5 \mathrm{M} \mathrm{CaCl}_{2}$ stock solution and $50 \mu \mathrm{l}$ of $2 \mathrm{X}$ BBS-buffered saline (50 $\mathrm{mM} \operatorname{BBS}(\mathrm{N}, \mathrm{N}$-bis[2-hydroxyethyl]-2-aminoethanesulfonic acid), $280 \mathrm{mM} \mathrm{NaCl}, 1.5 \mathrm{mM}$ $\left.\mathrm{Na}_{2} \mathrm{HPO}_{4}, \mathrm{pH} 7.05\right) \mathrm{kept}$ at room temperature in darkness for $15-20 \mathrm{~min}$. The precipitate was added drop-wise to $900 \mu \mathrm{NBA}$ while gently vortexing. $500 \mu \mathrm{l}$ of the diluted calcium phosphate/DNA precipitate solution was then added to cells in each well after removing the medium. After 20 min incubation at $37^{\circ} \mathrm{C}$ and $5 \% \mathrm{CO}_{2}$, a fine sandy precipitate covering the cells was observed. The cultures were then washed in Hank's Balanced Salt Solution (HBSS; 
Invitrogen) and finally replaced with the original growth media. The efficiency was typically between $5 \%$ and $20 \%$, and there was no apparent toxicity to the cells. GFP expression could be detected as early as 6 hrs post-transfection and remained stable for several weeks. Imaging was performed at 14-21 DIV.

\subsection{Synaptobrevin 2/ Cellubrevin mutant mice}

Syb2 and Ceb mutant mice were kept in different colonies. Heterozygote of Ceb was maintained by continuous crossbreeding with C57BL/6 and Ceb KO animals, which are viable and fertile. Heterozygous mice for Syb2 and homozygous KO mice for Ceb were obtained by crossbreeding of the mutant Syb2 and Ceb mouse strains. The DKO mice were created by crossing of heterozygous Syb2 and KO Ceb mouse strains and confirmed by standard PCR genotyping as described previously (Schoch et al., 2001). These animals die at birth and exhibit a phenotype similar to the Syb2 KO phenotype, (Borisovska et al., 2005; Schoch et al., 2001) with a tucked posture and no spontaneous movements or sensorimotor reflexes (Borisovska et al., 2005). All analyses were performed on E18 animals derived from the heterozygote matings.

\subsection{Fluorescent probes}

Due to the small size $(\sim 1 \mu \mathrm{m})$ of bouton-type synapses, they have mostly been inaccessible to electrophysiological techniques. Therefore optical tools based on fluorescent membrane probes have been used to analyze presynaptic function. Fluorescent membrane tracers have enabled quantitative measurments of SV exo-endocytosis at individual synapses and even at a single molecule level. In this study two different kinds of fluorescent probes have been used a) genetically encoded probes such as fusion chimeras of GFP variants with specific SV protein of interest and b) exogenous tracer based on cyanine dye derivative, cypHer5E coupled to antibodies against specific SV markers. 


\subsubsection{Overexpression of pHluorin-tagged SV proteins}

The excitation spectrum of WT GFP is not sensitive to changes in the $\mathrm{pH}$ of the environment. A systematic histidine-based combinatorial mutagenesis of key residues resulted in the generation of a novel pH-sensitive GFP mutant termed 'ecliptic pHluorin' (e-pHI) which exhibited $\mathrm{pH}$-dependent absorbance characteristics. At a $\mathrm{pH}$ of $<6$, the excitation peak at 475 $\mathrm{nm}$ of e-pHI disappears and it ceases to be fluorescent, thus 'eclipsed'. Enhanced GFP (EGFP), which is several fold brighter and contains a single excitation peak at $488 \mathrm{~nm}$, also exhibits some $\mathrm{pH}$-dependence, although the dynamic range of the $\mathrm{pH}$-dependent fluorescence change is much smaller (Ashby et al., 2004). Subsequently, e-pHI was further mutated to generate a superecliptic $\mathrm{pHI}(\mathrm{pH})$, which is $\sim 9$ times brighter and displays the same $\mathrm{pH}$ sensitivity. The $\mathrm{pH}$-dependent fluorescent property of $\mathrm{pH}$ is described by a single protonbinding equilibrium, where the protonated state is the non-fluorescent state (Palm et al., 1997; Sankaranarayanan et al., 2000). Thus, the baseline fluorescence is determined by the Henderson-Hasselbach equation, where the fraction of deprotonated $\mathrm{pHI}$ molecules $[X]$ is described by

$$
[X]=\frac{1}{1+10^{\mathrm{pK}-\mathrm{pH}}}
$$

where $\mathrm{pK}$ is the logarithm of the equilibrium constant for protonation. During fusion and increase in $\mathrm{pH}$ from 5.5 from 7.4 , the change in $[X]$ will be

$$
\Delta[X]=\frac{1}{1+10^{\mathrm{p} K-7.4}}-\frac{1}{1+10^{\mathrm{p} K-5.5}}
$$

Thus, pK determines the magnitude of this signal.

By genetically fusing e-pHI or $\mathrm{pHI}$ to the luminal domain of Syb2 $(\mathrm{SpH})$, they can be targeted to SVs, where the fluorescence is quenched due to the acidic lumen ( $\mathrm{pH}$ 5.5). Upon SV fusion the $\mathrm{pH}$ is neutralized resulting in deprotonation of the $\mathrm{pHI}$ and a dramatic increase in its fluorescence. The $\mathrm{pK}$ for $\mathrm{pHI}$ in overexpressed neurons is $\sim 7.1$ (Fig. 2.1b). The fluorescence change for $\mathrm{pH}$ upon fusion is $\sim 20-25$ times (from $\mathrm{pH} 5.5$. to 7.4 ) and therefore ideal as a pH- 
sensor. When overexpressed in hippocampal neurons in culture, $\mathrm{SpH}$ exhibits a diffuse distribution both in boutons and at the axonal regions with a significant fraction targeted to the plasma membrane (Fig. 2.1a). This surface fraction varies among different $\mathrm{pH}$ fusion proteins with SV proteins such as Syp-pHI, Syt1-pHI, and vGlut-pHI. Upon extracellular stimulation, the fluorescence increases due to deprotonation of $\mathrm{pHI}$ upon exocytosis and $\mathrm{pH}$ neutralization. Subsequently, the fluorescence signal slowly returns to baseline due to endocytosis and reacidification, which can be well described by a monoexponential recovery. The kinetics of recovery represents the time course of endocytosis since reacidification has been shown to occur at a relatively fast time scale $(\sim 4 \mathrm{~s})$ (Sankaranarayanan and Ryan, 2000). Typical pHI fluorescence transients upon stimulation-dependent exo-endocytosis have been shown in Fig 2.1d. The amplitude of fluorescence change scales with the strength of stimulation implying that with increasing stimulation more SVs are recruited until a point, where the entire SV recycling pool of these synapses has been depleted. Due to the low $P_{r}$ of hippocampal synapses and optimal signal-to-noise ratio of the $\mathrm{pHI}$ probes, it is possible to elicit and optically resolve single SV fusion. 
(a)

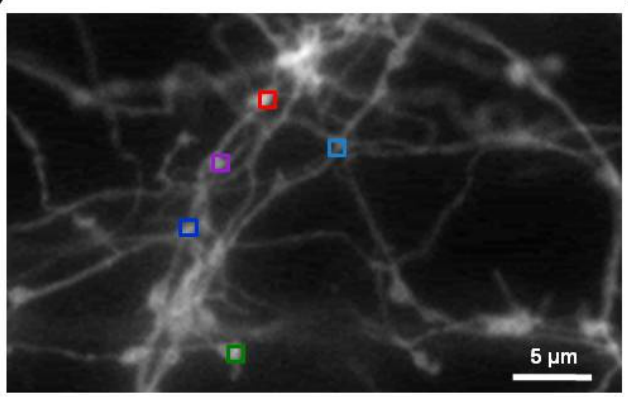

(c)

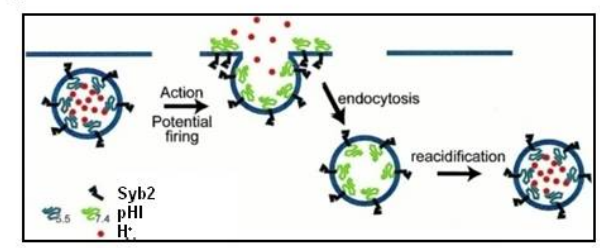

(b)

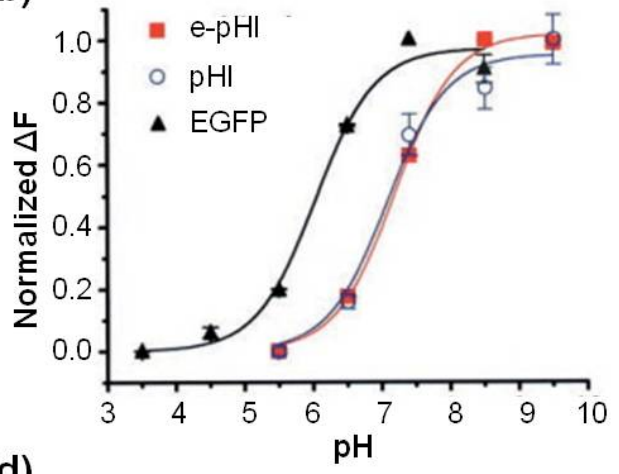

(d)

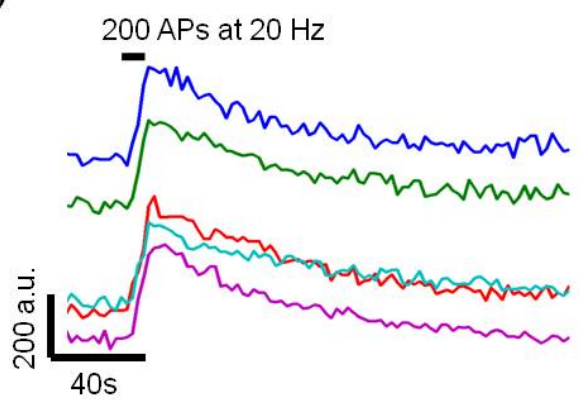

Figure 2.1: $\mathrm{SpH}$ is a reliable reporter for measuring exo-endocytosis.

(a) Exemplar image of hippocampal neurons transfected with $\mathrm{SpH}$ (Scale bar: $5 \mu \mathrm{m}$ ).

(b) $\mathrm{pH}$ titration curve of e-pHI, $\mathrm{pHI}$ and EGFP with a $\mathrm{pK}$ of $7.07,7.18$ and 6.03 ideal for measuring exoendocytosis. Taken from (Sankaranarayanan et al., 2000).

(c) Resting SVs are at an acidic $\mathrm{pH}(\mathrm{pH} 5.5)$, which quenches the luminal $\mathrm{pHI}$. Upon fusion and neutralization with the extracellular $\mathrm{pH}$, the proton-dependent quenching is relieved and the fluorescence signal increases. Upon endocytosis and reacidification, the fluorescence slowly recovers. Taken from (Sankaranarayanan et al., 2000).

(d) Exemplar fluorescence responses from putative boutons marked in (a) upon field stimulation. The rising phase indicates externalization of $\mathrm{SpH}$ and is followed by monoexponential recovery due to endocytosis and reacidification.

In the entire study five different variants of pHI-tagged SV proteins have been used:

SpH (Miesenbock et al., 1998; Wienisch and Klingauf, 2006) was kindly provided by G. Miesenböck (Yale University, New Haven). Another variant of $\mathrm{SpH}, \mathrm{SpH}-\mathrm{TEV}$ was used in the second part of the study where a Tobacco Etch Virus (TEV) protease recognition sequence was introduced flanked by flexible linker regions in between Syb2 and pHI was introduced. 
Syp-pHI (Granseth et al., 2006) was provided by Leon Lagnado (MRC, Cambridge, UK) and vGlut-pHI (Balaji and Ryan, 2007) was a gift from Robert Edwards (UCSF, San Francisco). The fusion constructs Syt1-pHI, Syp-pHI and vGlut-pHI were driven by the ubiquitous Cytomegalovirus (CMV) promoter where as $\mathrm{SpH}$ was driven by a neuron-specific human Synapsin-1 gene promoter (Wienisch and Klingauf, 2006) and verified by dideoxynucleotide sequencing.

\subsubsection{Live-cell immunolabeling with cypHer5E-coupled antibodies}

Overexpression of pHI-tagged SV proteins has been argued to cause artifacts and exhibit a different distribution pattern as compared to the endogenous proteins although presynaptic function remains perfectly normal (Opazo et al., 2010). To circumvent these drawbacks, in the second part of the study, a novel exogenous tracer based on a $\mathrm{pH}$-sensitive cyanine dye derivative, CypHer5E conjugated to antibodies against endogenous proteins Syt1 and VGAT (aSyt1-cypHer and aVGAT-cypHer ; Synaptic Systems, Göttingen, Germany), was used to label hippocampal neurons and study SV recycling. Syt1 is an integral membrane glycoprotein with a single membrane spanning domain. It consists of a short $\mathrm{N}$-terminus at the luminal or extracellular side and a large conserved cytoplasmic $\mathrm{C}$-terminus domain with two $\mathrm{Ca}^{2+}$ binding C2 domains connected by a linker. VGAT is predicted to have 10 TMDs and a long $\mathrm{N}$-terminal cytoplasmic domain (for review see (Reimer et al., 1998)). The epitope for the cypHer-coupled antibody is at the luminal/extracellular side for both Syt1 (N-terminus) and VGAT (C-terminus) conducive for monitoring changes in $\mathrm{pH}$ during exo-endocytosis.

Neurons were incubated with either aSyt1-cypHer or aVGAT-cypHer, at $37^{\circ} \mathrm{C}$ for $3-4$ hrs in a bicarbonate buffer ( pH 7.4) containing $120 \mathrm{mM} \mathrm{NaCl}, 5 \mathrm{mM} \mathrm{KCl}, 1 \mathrm{mM} \mathrm{MgCl}, 2.5 \mathrm{mM}$ $\mathrm{CaCl}_{2}, 10 \mathrm{mM}$ glucose and $18 \mathrm{mM} \mathrm{NaHCO}_{3}$. The cells were then washed twice and placed in a perfusion chamber containing modified Tyrode solution $(150 \mathrm{mM} \mathrm{NaCl}, 4 \mathrm{mM} \mathrm{KCl}, 1 \mathrm{mM}$ $\mathrm{MgCl}_{2}, 2 \mathrm{mM} \mathrm{CaCl}$, $10 \mathrm{mM}$ glucose, $10 \mathrm{mM}$ HEPES buffer $\sim \mathrm{pH} 7.4$ ). Acidic buffer with a final $\mathrm{pH}$ of 5.5 and 6.5 was prepared by replacing HEPES with 2-[N-morpholino] ethane sulphonic acid] (MES; pK $=6.1$ ), whereas alkaline solution with $\mathrm{pH} 8.5$ and 9.5 was prepared by replacing HEPES with Bicine ( $\mathrm{pK}=8.5$ ), all other components remained unchanged. 
For $\mathrm{pH}$ titration measurements of cypHer, aSyt1-cypHer labeled neurons were stained, washed and then fixed with $4 \%$ paraformaldehyde (PFA) in phosphate buffered saline (PBS). Cells were permeabilized with $0.4 \%$ saponin to make the internalized antibodies accessible to perfusion with buffers of different $\mathrm{pH}$.

The surface pool staining for Fig. 3.21 and Fig. 3.22 was performed by incubating the neurons with high concentrations of aSyt1-cypHer (1:50) in the presence of $0.5 \mu \mathrm{M}$ tetrodotoxin (TTX) for $5 \mathrm{~min}$ at room temperature. The neurons were then washed with normal Tyrode solution and used for imaging.

\subsection{Tobacco etch virus protease cleavage}

Tobacco etch virus (TEV) encodes a $27 \mathrm{kDa}$ catalytic domain of the Nuclear Inclusion a (NIa) protein called the TEV protease. Due to its strict sequence specificity compared to other proteases, such as factor $\mathrm{Xa}$, thrombin, or enterokinase, TEV protease is very useful for effectively cleaving fusion proteins. TEV protease recognizes a linear epitope of the general form E-Xaa-Xaa-Y -Xaa-Q-(G/S), with cleavage occurring between $Q$ and $G$ or $Q$ and $S$ (Parks et al., 1995). The most commonly used sequence is ENLYFQG. Unlike other proteases such as thrombin, TEV proteases do not have a secondary protease activity or serve as agonist for proteinase-activated receptors expressed in neurons which can alter the physiological function and even lead to cell death (Donovan et al., 1997; Kiseleva et al., 2004; Smith-Swintosky et al., 1997).

SpH-TEV transfected neurons were incubated with AcTEV protease and $1 \mathrm{mM}$ dithiothreitol (both from Invitrogen) for $15 \mathrm{~min}$ at room temperature as described previously (Wienisch and Klingauf, 2006). After digestion, neurons were washed several times with normal Tyrode solution to remove the enzyme and cleaved $\mathrm{pHI}$. Cleavage of surface $\mathrm{SpH}$ was observed over time as a reduction in the fluorescence signal. 


\subsection{Optical Imaging}

Coverslips were placed in a perfusion chamber $(\sim 500 \mu$ volume $)$ containing a modified Tyrode solution (in mM: $140 \mathrm{NaCl}, 5 \mathrm{KCl}, 2 \mathrm{CaCl}_{2}, 2 \mathrm{MgCl}_{2}, 30$ Glucose, 10 HEPES, pH 7.4; 330 mOsm). APs were elicited by electric field stimulation with $1 \mathrm{~ms}$ pulses of $50 \mathrm{~mA}$ generated by a constant current stimulus isolator (WPI A 385, World Precision Instruments) between platinum-iridium electrodes (distance $\sim 1 \mathrm{~cm}$ ) in the presence of 6-cyano-7-nitroquinoxaline2,3-dione (CNQX; $10 \mu \mathrm{M}$; Tocris Bioscience) and D-amino-5-phosphonovaleric acid (D,LAPV; $50 \mu \mathrm{M}$; Tocris Bioscience) to prevent recurrent activity. Given the low $\mathrm{P}_{\mathrm{r}}$ of hippocampal synapses, single vesicle fusion was evoked by eliciting 1 AP in Syp-pHI, vGlut-pHI and SpH measurements. Bulk measurements to calculate the size of the recycling pool of SVs was performed by eliciting $900 \mathrm{APs}$ at $20 \mathrm{~Hz}$ in presence of $65 \mathrm{~nm}$ folimycin (Calbiochem). Total pool size was calculated by dequenching the residual $\mathrm{SpH}$ in the bouton after 900 APs stimulation with $\mathrm{NH}_{4} \mathrm{Cl}$ solution, which was prepared by substituting $50 \mathrm{mM} \mathrm{NaCl}$ in normal saline with $\mathrm{NH}_{4} \mathrm{Cl}$, while the remaining constituents were unchanged.

\subsection{Single vesicle optical recordings with pHluorin-based probes}

Experiments were conducted at room temperature on an inverted Nikon TE2000 microscope equipped with a 100X/1.45 numerical aperture (NA) oil immersion objective (Nikon, Tokyo, Japan). The $\mathrm{pH}$ fusion constructs were excited at $488 \mathrm{~nm}$ by a mechanically shuttered Argon laser whose beam was slightly defocused at the back focal plane to fill the field of view (17 $\mathrm{mW}$ at the back-focal plane). Images (128 X 128 pixels) were acquired with a back-illuminated electron multiplying charge couple device (EMCCD) camera (DV-860 camera; Andor Technology, Belfast, UK) at $100 \mathrm{~Hz}$ frame rate using $9.6 \mathrm{~ms}$ exposure time during short $0.5 \mathrm{~s}$ periods of constant laser illumination to minimize photobleaching. The stimulus was applied at $0.15 \mathrm{~s}$, and the sequence was repeated 10 times with an interval of $10 \mathrm{~s}$ in between consecutive trials. On average 10-20 boutons were imaged from one field of view on one coverslip due to the small chip size of the camera (DV-860 camera) used. For both WT and DKO SpH recordings, the data has been compiled from 5-6 independent transfections of hippocampal neurons prepared from 8-9 individual E18 pups, which were later reconfirmed by 
genotyping. For single vesicle recordings with $\mathrm{SpH}$-transfected boutons, imaged regions were pre-photobleached for $50 \mathrm{~s}$, using the same laser intensity (17 $\mathrm{mW}$ at back-focal plane) as during image acquisition.

\section{8 pHluorin and cypHer measurements (bulk stimulation)}

Fluorescence measurements of $\mathrm{SpH}$-expressing boutons for $40 \mathrm{APs}$ and $100 \mathrm{APs}$, were performed at reduced laser intensity ( $4 \mathrm{~mW}$ at back-focal plane) and at a $2 \mathrm{~Hz}$ image acquisition frequency. The rest of the $\mathrm{SpH}, \mathrm{Syt1}-\mathrm{pHI}$ and cypHer bulk stimulation experiments were performed with a 60X, 1.2 NA water-immersion objective. Images (512 X 512 pixels) were acquired with a back-illuminated EMCCD camera (Andor iXon+ DU-897E-BV camera; Andor Technology, Belfast, UK) controlled by Andor iQ Software (Andor Technology, Belfast, UK). $\mathrm{pH}$ and cypHer was excited at $488 \mathrm{~nm}$ and $640 \mathrm{~nm}$ respectively, with a computercontrolled monochromator (Polychrom IV, Till Photonics) and imaged using an EGFP single band or EGFP/Cy5 dual-band filter set (AHF Analysentechnik AG, Tuebingen). Time-lapse images were acquired at 0.2 and $0.5 \mathrm{~Hz}$ sampling frequency using an exposure time of 200 ms. Due to the larger chip size and lower magnification of the objective lens, more than 100 boutons could be detected per field of view for $\mathrm{SpH}$ overexpression in WT and DKO neurons. Fast solution exchanges were achieved through a two-barrel glass tubing perfusion system controlled by a piezo-controlled stepper device (SF778, Warner Instruments). Dual-color recordings of $\mathrm{SpH}$ (green) and cypHer (red) were acquired at $0.5 \mathrm{~Hz}$ one after the other. Vesicular cypHer was photobleached by continuous exposure to red light $(640 \mathrm{~nm})$ for $5 \mathrm{~min}$. During the cypHer bleaching procedure (Fig. 3.20), cells were bathed in an alkaline buffer solution of pH 8.5 for better protection of surface bound cypHer molecules.

\subsection{Single-molecule pHluorin experiments}

The $\mathrm{pH}$ expression construct was designed and generated together with Entelechon (Regensburg, Germany). In brief, the SpH construct (Wienisch and Klingauf, 2006) was used as a template to introduce suitable $5^{\prime}$ and $3^{\prime}$ ends by PCR amplification for subsequent cloning into the $\mathrm{pET} 21 \mathrm{~b}(+)$ expression vector. A best clone screen was performed to identify the E. coli clone with the highest protein expression. $100 \mu \mathrm{g}$ of the $\mathrm{pHI}$ protein was purified 
and dissolved in $10 \mathrm{mM}$ Tris, $10 \mathrm{mM}$ Ethylenediaminetetraceticacid (EDTA; pH 8) with a final concentration of $1 \mu \mathrm{g} / \mu \mathrm{l}$. The purified protein was immobilized in a polyacrylamide gel (Kubitscheck et al., 2000). Experiments were performed using the same laser intensity and camera settings as in the neuronal experiments for single vesicle detection. Images were acquired at $15 \mathrm{~Hz}$ (9.6 ms exposure). Square regions of interest (ROls; 1 by $1 \mu \mathrm{m}$ ) were overlaid on fluorescence spots and mean intensities were plotted. Single molecule fluorescence amplitude was calculated by subtracting an average of 5-10 frames before and after the bleaching step. Extrapolating from the measured photobleaching time constants at $\mathrm{pH} 7.4$ and 9 we estimated that 50 s surface fluorescence prebleaching results in up to $20 \%$ bleaching of the vesicular fraction of $\mathrm{SpH}$ at a $\mathrm{pH}$ of 5.5.

\subsection{Image and data analysis}

Data were acquired using the Andor IQ software suite (Andor Technology, Belfast, UK). Quantitative analysis was performed with MetaMorph 6.0 (Molecular devices, USA) and with self-written macros in Igor Pro 6.03A (WaveMetrics Inc, Lake Oswego, OR, USA). To avoid the bias introduced by manual selection of functional boutons, an automated detection algorithm was used to localize the active boutons. The difference image, generated by subtracting an average of 5 images in the time series before and after stimulation, was subjected to an à trous wavelet transformation with the level $k=4$ and detection level $I_{d}=1.0$, resulting in a segmented mask image (Wienisch and Klingauf, 2006). Spots on the mask image, each representing putative functional boutons, were identified, and their co-ordinates were used to overlay a square ROI (as mentioned above) on the original image series. Only masks with areas between 4 and 16 pixels were accepted for localizing the bouton fluorescence transients. All identified masks were visually inspected for correspondence to individual functional boutons. Mean fluorescence intensities from the ROls were then plotted. $\Delta \mathrm{F}$ was calculated as the difference in average intensity of five frames before and after stimulation (average peak fluorescence). Responses from all 10 trials of the experiments (including failures), for each detected bouton, was used to build the $\Delta \mathrm{F}$ histograms in the case of $\mathrm{SpH}$ and Syt1-pHI. However, for amplitude distributions of vGlut-pHI and Syp-pHI a fraction 
of the failures (less than $1 \sigma$ of the quantal size) were used to get a resulting noise peak in the histograms for fitting. Therefore, vGlut-pHI and Syp-pHI intensity histograms should not be used as a measure of $\mathrm{P}_{\mathrm{r}}$.

Images from cypHer measurements were analyzed using custom-written routines in Matlab (MathWorks). Active boutons were localized based on $\mathrm{SpH}$ fluorescence using an automated routine where a difference image is generated by subtracting an average of 5 frames before from after stimulation. Spots on the difference image, each representing putative functional boutons, were identified and centered based on the maximum pixel intensity. Only spots with fluorescence response $>2 \sigma$ of baseline fluorescence were chosen for analysis. Square ROls $\left(1.5 \times 1.5 \mu \mathrm{m}^{2}\right)$ were centered on these spots in both the $\mathrm{SpH}$ and cypHer channel and average fluorescence profiles were then plotted. Due to the low transfection efficiency of $\mathrm{SpH}$, not every cypHer positive bouton was $\mathrm{SpH}$ positive. For dual-color recordings, we used the coordinates of the ROIs identified from $\mathrm{SpH}$ response to define the positions of active boutons in the cypHer channel. Fluorescence traces from individual boutons were normalized before calculating the average response.

\subsection{Data fitting}

The $\Delta \mathrm{F}$ histograms of single AP responses from $\mathrm{SpH}$, Syt1-pHI, Syp-pHI and vGlut-pHI transfected boutons were fit to a multimodal Gaussian distribution constrained by the quantal size q (width of each peak) and coefficient of variation for the intensity measurements $c_{m}$ based on previously described procedures (Gandhi and Stevens, 2003; Lemke and Klingauf, 2005; Murthy and Stevens, 1998):

$$
\sum_{k=0}^{n} A_{k} \cdot \exp \left(-\frac{1}{2} \frac{[x-(q \cdot k)]^{2}}{c_{m}^{2}\left[(q \cdot k+r)^{2}+r^{2}\right]}\right)
$$

where $A_{k}$ is the amplitude (number of responses) of the $k^{\text {th }}$ peak, $q$ is the average intensity of an individual quantum (peak distance in the fit), $c_{m}$ is the coefficient of variation of the quantal intensity and $r$ is the baseline/background fluorescence. The positions of each peak were constant and evenly spaced at integer multiples of q. Due to the smearing of the zero-order 
peak in the negative direction only a sub-range of the entire histogram was fit as indicated by the overlaid best-fit curves. All Gaussian curve fittings were performed using LevenbergMarquardt $x^{2}$ minimization for non-linear least-square fitting. The baseline fluorescence $r$ was taken as the average camera readout before stimulation and represents the background noise of our measurements which consists of a mixture of camera shot noise, laser-illumination noise, the signal from unquenched pHI-tagged molecules on the surface and the quenched $\left(1 / 25^{\text {th }}\right)$ fluorescence of acidified SVs. This baseline fluorescence $r$ determines the standard deviation of the $k^{\text {th }}$ peaks. All other parameters were freely fit.

The in vitro single molecule histograms were fit using a multigaussian function as described below :

$$
\sum_{k=0}^{n} A_{k} \cdot \exp \left(-\frac{\left[x-\mu_{k}\right]^{2}}{2 \sigma_{k}^{2}}\right)
$$

where the parameters of the function i.e. amplitude $\left(A_{k}\right)$, peak spacing $\left(\mu_{k}\right)$ and standard deviation $\left(\sigma_{k}\right)$ are freely fit. Since the quantal size corresponds to single $\mathrm{pHI}$ molecules the zero and first-order peaks in the in vitro experiments are comparable to that of the single AP fluorescence responses and hence represent the true resolution of the optical measurements without any smearing in the negative direction of the zero order peak, as explained before. From these in vitro distributions we can derive the standard deviation of the kth peaks based on the following equation as previously described (Gandhi and Stevens, 2003).

$$
\sigma_{k t h p e a k}=\sqrt{\sigma_{0}^{2}+|k| \sigma_{q}^{2}}
$$

Based on the in vitro single molecule distributions we could empirically determine $\sigma_{q}$ and hence predict the standard deviations of the higher-order peaks in the single AP amplitude distributions. The computed standard deviations were comparable to those estimated from the Gaussian fitting of individual higher-order peaks for the single AP SpH distribution (Fig. S1). The standard deviations do increase with an increase in the mean $\Delta \mathrm{F}$ amplitude as expected from Poissonian statistics, but this peak broadening is not very prominent since the baseline 
fluorescence $r$ and $\sigma_{0}$ are relatively high and hence dominate the $\sigma$ of the kth peaks (Fig. S1). We circumvented this problem by averaging fluorescence intensity over 5 frames and over 16 pixels ( $4 \times 4$ pixels).

\subsection{Lentiviral transduction and immunoblotting}

The lentivirus plasmid expressing $\mathrm{pH}$ fused to the C-terminus of Syb2 was used to infect hippocampal neuronal cultures at DIV 1. The neurons were lysed and harvested at DIV 12 using $0.1 \mathrm{ml}$ of lysis buffer containing $150 \mathrm{mM} \mathrm{NaCl}, 10 \mathrm{mM}$ Hepes (pH 7.4), 1 mM EGTA, 2 $\mathrm{mM} \mathrm{MgCl} 2,1 \%(\mathrm{v} / \mathrm{v})$ Triton X-100 and protease inhibitor cocktail tablet (Roche, Applied Science). The lysates were centrifuged at 8,000 rpm for $5 \mathrm{~min}$. The supernatant was collected, denatured and separated in $12 \%$ SDS-PAGE followed by western blotting using standard procedures. The blot was probed with a mouse monoclonal antibody against Syb2 (69.1, 1:6,000, Synaptic Systems, Göttingen, Germany) followed by detection with an anti-mouse secondary antibody coupled to horseradish peroxidase. The blot was developed by enhanced chemiluminescence (PerkinElmer Life Sciences) and detected using a LAS 1000 CCD camera (Fugifilm). To show equal loading of each lane, the membranes were washed in stripping buffer, rinsed, and reprobed with polyclonal antibody against lactate dehydrogenase (LDH; SC-33781, Santa Cruz Biotechnology). 


\section{RESULTS}

\subsection{Number of SNARE complexes necessary and sufficient for evoked fusion}

\subsubsection{Optical recording of single vesicle fusion}

As a first step to quantify the number of SNARE molecules, it is crucial to measure fusion of single SVs upon stimulation. pHI fusion chimeras have been used successfully to monitor exoendocytosis, however it has been challenging to detect single exocytic events. When $\mathrm{pHI}$ is fused to the luminal domain of SV proteins its fluorescence is quenched at the acidic intravesicular $\mathrm{pH}$ of $\sim 5.5$, but its fluorescence increases about 25 -fold when exposed to the neutral extracellular $\mathrm{pH}$ during exocytosis (Miesenbock et al., 1998). The resolution of such measurements is primarily limited by background fluorescence caused by a fraction of $\mathrm{pHI}$ tagged SV proteins targeted to the presynaptic membrane. The size of this surface pool varies for different SV proteins and has been shown to actively participate in exo-endocytic cycling (Fernandez-Alfonso et al., 2006; Wienisch and Klingauf, 2006). While this surface pool is small for vGlut-pHI and Syp-pHI with 2-3 \% for vGlut-pHI (Balaji and Ryan, 2007) and 9 \% for Syp-pHI (Granseth et al., 2006), it is very high for SpH and Syt1-pHI with up to $30 \%$ and $24 \%$ respectively (Gandhi and Stevens, 2003; Wienisch and Klingauf, 2006). Thus, single vesicle fusion events could be easily visualized using vGlut-pHI (Balaji and Ryan, 2007) or Syp-pHI (Granseth et al., 2006) as reporters (Fig. 3.1), but only one study so far has reported single vesicle resolution for viral overexpression of $\mathrm{SpH}$ (Gandhi and Stevens, 2003), the protein of interest here. 
(a)

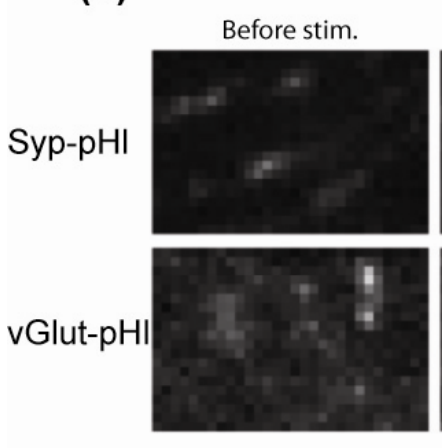

After stim.

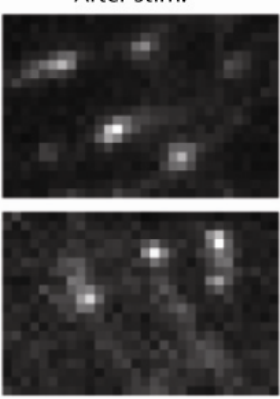

Difference.
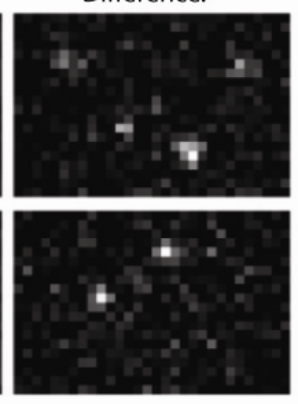

(b)

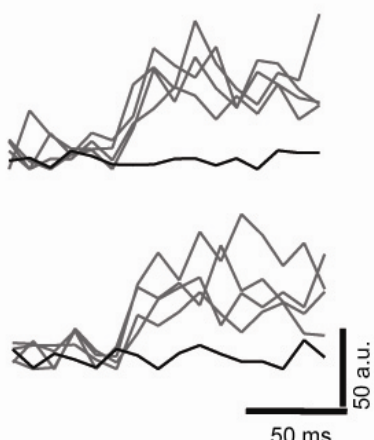

Figure 3.1: Optical detection of single SV fusion events.

(a) Image of Syp-pHI and vGlut-pHI transfected boutons before (Before Stim.) and after (After Stim.) 1 AP stimulation. Difference image reveals sites of evoked release.

(b) Exemplar fluorescence traces from individual boutons exhibit instantaneous increase upon SV fusion.

The SpH background fluorescence, however, can be selectively attenuated by prephotobleaching, since the quenched intravesicular $\mathrm{SpH}$ is mostly protected against bleaching (Gandhi and Stevens, 2003). If bleaching is executed fairly rapidly, relative to spontaneous SV fusion and recycling, the surface pool is mostly bleached, and exo- and endocytic trafficking of SpH is normal (Fig. 3.2) (Wienisch and Klingauf, 2006). To eliminate this background fluorescence and achieve optimal signal-to-noise ratio for single vesicle detection, the neurons were prebleached for 50 s with continuous laser illumination at $488 \mathrm{~nm}$ (Fig. 3.2). The size of surface and vesicular fraction of $\mathrm{SpH}$ was measured with the sequential application of acid and alkaline solution before and after bleaching (Fig. 3.2). The acid (pH 5.5) pulse selectively quenches the fluorescence of the surface pool of $\mathrm{SpH}$ denoted by a decrease in fluorescence, which recovers upon reperfusion with a neutral $\mathrm{pH}$ solution (Fig. 3.2). Subsequently, when neurons were challenged with an $\mathrm{NH}_{4} \mathrm{Cl}$ pulse, which neutralizes $\mathrm{pH}$ across all membranes and thereby unmasks all intracellular $\mathrm{SpH}$, the fluorescence increased dramatically giving an estimate of the total vesicular SpH (Fig. 3.2). By comparing the two average fluorescence profiles before and after bleaching, it was estimated that the surface 
$\mathrm{SpH}(\sim 90 \%)$ was selectively bleached whereas the vesicular fraction remained largely unaffected (Fig. 3.2).

(a)

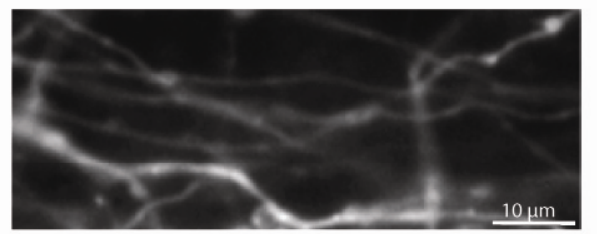

(iii)

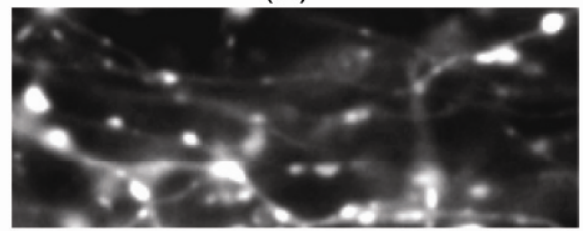

(b)

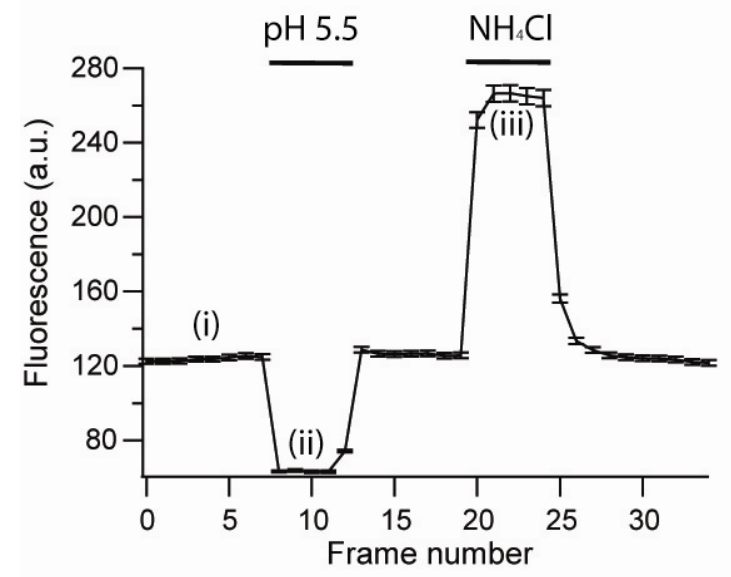

(ii)

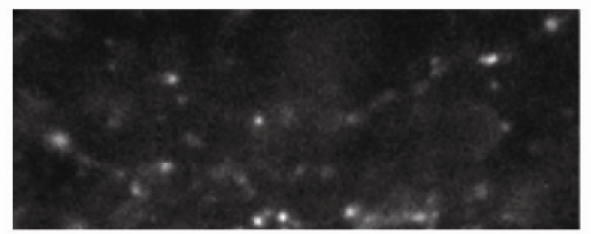

After Bleaching

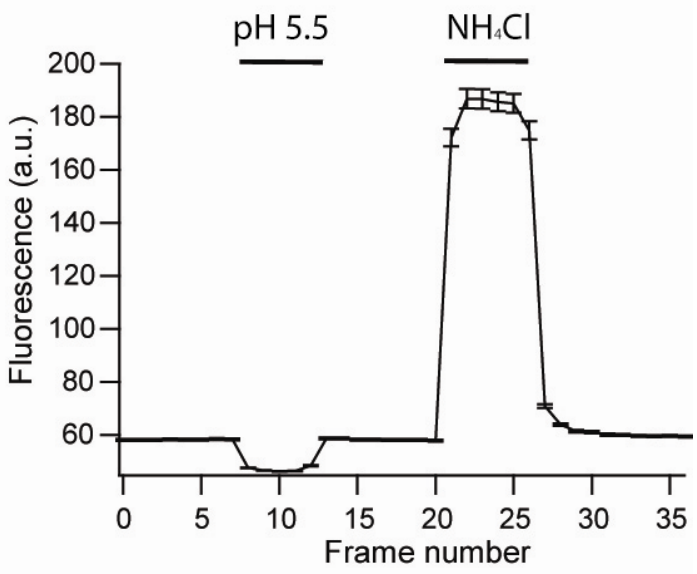

Figure 3.2: Prebleaching selectively attenuates the surface-stranded $\mathrm{SpH}$ without affecting the vesicular $\mathrm{SpH}$ fraction.

(a) Fluorescence images of $\mathrm{SpH}$-transfected Syb2/Ceb DKO neurons during the sequential pulses of an acidic solution and $\mathrm{NH}_{4} \mathrm{Cl}$ solution.

(b) Exemplar average fluorescence traces before and after laser illumination for $50 \mathrm{~s}$ clearly demonstrate that $\sim 90 \%$ of the surface $\mathrm{SpH}$ molecules are photobleached and only $\sim 10-15 \%$ of the vesicluar $\mathrm{SpH}$ fraction is affected ( $n>1000$ boutons from 4 experiments). Error bars indicate s.e.m. 
With prebleaching the surface pool for $50 \mathrm{~s}$, I could indeed image single SV fusion events in synaptic boutons of SpH-expressing WT hippocampal neurons in culture, when stimulated by single APs (Fig. 3.3a). To obtain robust fluorescence responses without appreciable photobleaching, the sampling rate was increased to $100 \mathrm{~Hz}$ and the time window for imaging and illumination was minimized to $0.5 \mathrm{~s}$. Under these experimental conditions, $\mathrm{SpH}$ fluorescence responses from individual boutons exhibited instantaneous increases upon stimulation, indistinguishable from responses obtained from boutons expressing either SyppHI or vGlut-pHI (Fig. 3.3b, Fig. 3.1).

(a)

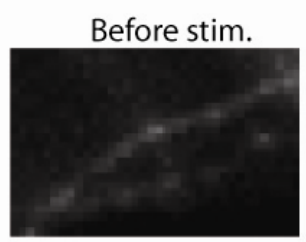

After stim.

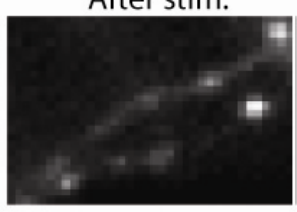

Difference.

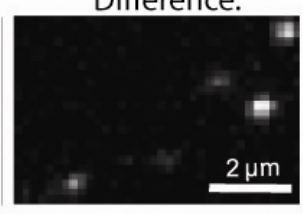

(b)

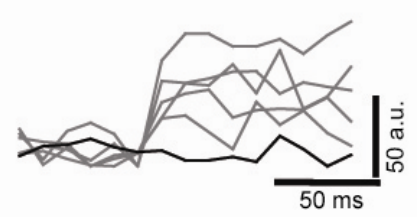

Figure 3.3: Prebleaching enables detection of single fusion events in $\mathrm{SpH}$ transfected boutons.

(a) Exemplar image of $\mathrm{SpH}$ transfected boutons before and after $1 \mathrm{AP}$. Difference image reveals sites of evoked release.

(b) Fluorescence transients from individual boutons similar to Syp-pHI and vGlut-pHI responses in Fig 3.1. 


\subsubsection{Single action potential fluorescence responses display quantal distribution}

A histogram of the fluorescence responses, $\Delta \mathrm{F}$, at individual boutons upon stimulation was constructed (Fig. 3.4). The $\Delta \mathrm{F}$ distributions for all four reporters displayed several evenly spaced peaks (Fig. 3.4). The zero-order peak represented failures to evoke fusion and indicated the baseline variability of the measurements, while the higher order peaks represented release of one, two or more packages (quanta) of $\mathrm{pHI}$ molecules. These quanta might originate from the fusion of either single SVs, as shown in previous studies where lowering extracellular $\mathrm{Ca}^{2+}$ (which reduces $\mathrm{P}_{\mathrm{r}}$ ) reduced the frequency of the higher-order peaks, or alternatively from SVs with varying number of $\mathrm{pHI}$ molecules (Balaji and Ryan, 2007; Gandhi and Stevens, 2003; Granseth et al., 2006). To estimate the quantal size $q$, the fluorescence contribution of single fusing SVs, the histograms were fitted to multiple Gaussian curves (Material and Methods) (Lemke and Klingauf, 2005; Murthy and Stevens, 1998), which yielded very similar values for the reporters, namely $14.7 \pm 0.15$ arbitrary units (a.u.) for vGlut-pHI, $14.7 \pm 0.08$ a.u. for Syp-pHI, and $15.2 \pm 0.21$ a.u. for SpH (Fig. 3.4). Moreover, the quantal size remained invariant irrespective of the type of promoter used to drive expression (CMV promoter for ubiquitous Syp-pHI and vGlut-pHI expression and synapsin promoter for neuron-specific $\mathrm{SpH}$ expression). 

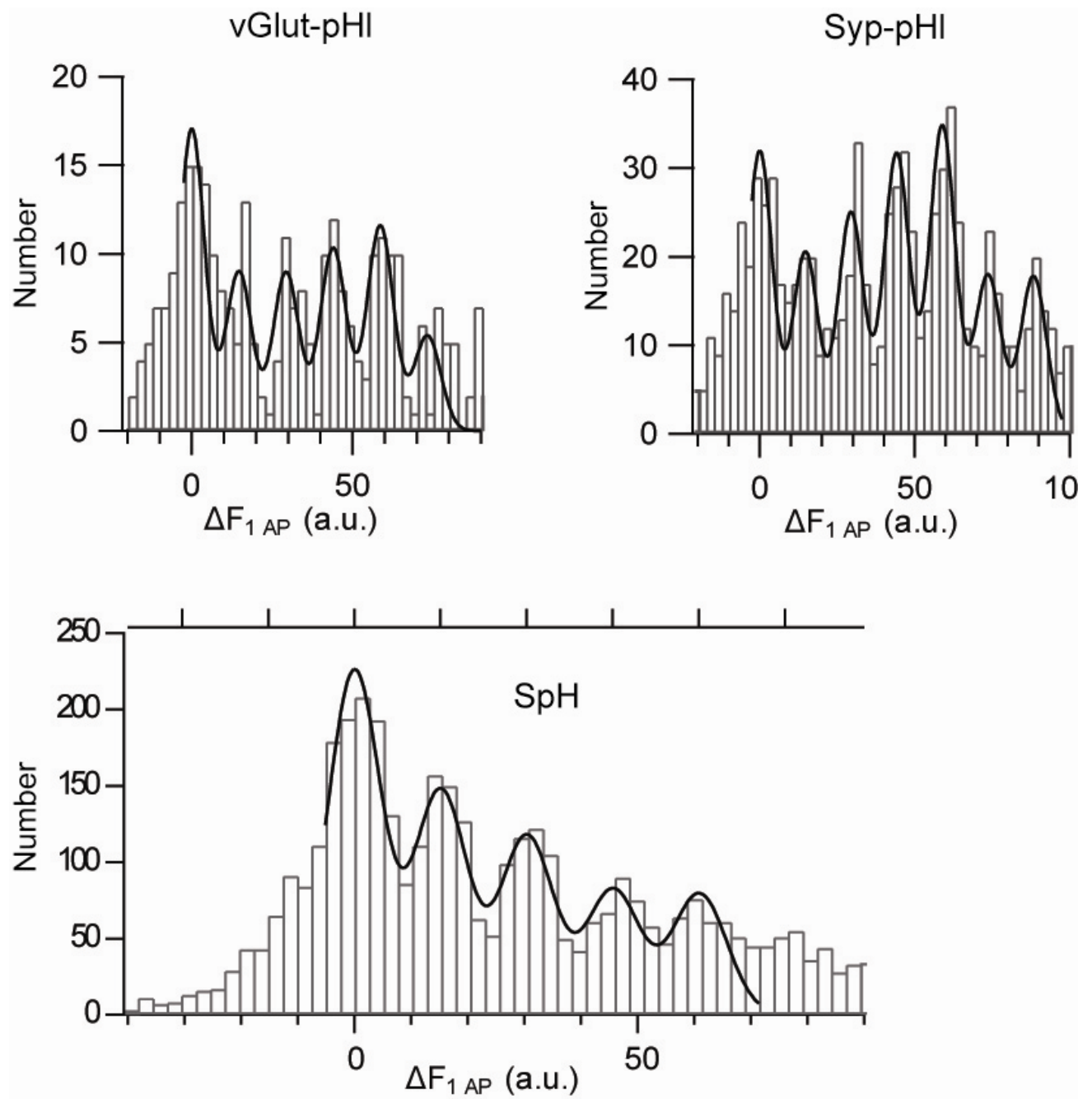

Figure 3.4: $\Delta \mathrm{F}$ distributions of single AP fluorescence responses appear quantized.

$\Delta \mathrm{F}$ distributions from vGlut-pHI, Syp-pHI and $\mathrm{SpH}$ overexpressing boutons evoked by 1 AP plotted with a binwidth of $2.5 \mathrm{a}$.u. The smooth solid curve is the best overall fit to multiple Gaussians of fixed quantal size $q \pm$ s.d. The estimated $q$ is $14.7 \pm 0.17$ a.u. for vGlut-pHI $(n>30$ boutons from 3 experiments, $14.7 \pm$ 0.08 a.u. for Syp-pHI ( $\mathrm{n}>100$ boutons from 6 experiments), and $15.2 \pm 0.21$ a.u for $\mathrm{SpH}$ ( $\mathrm{n}>400$ boutons from 14 experiments; adjusted $\mathrm{R}^{2}=0.8$ for both vGlut-pHI and Syp-pHI; adjusted $\mathrm{R}^{2}=0.94$ for $\mathrm{SpH}$ ). 


\subsubsection{Quantal size is equivalent to single pHluorin molecule fluorescence}

\subsection{3a Single molecule bleaching analysis of immobilized pHluorin}

To calibrate the quantal sizes in terms of number of $\mathrm{pHI}$-tagged proteins per SV, photobleaching experiments were performed using a dilute solution of immobilized $\mathrm{pHI}$ molecules on a coverslip (Kubitscheck et al., 2000). Isolated pHI molecules appeared as diffraction-limited spots, which were then bleached by constant irradiation with a $488 \mathrm{~nm}$ Argon laser (Fig. 3.5a). Single pHI molecules could be identified by photobleaching in single steps (Fig. 3.5b). The distribution of fluorescence downsteps at pH 9 was well described by a sum of two evenly spaced Gaussian curves with a mean size of $20.1 \pm 1.09$ a.u. (Fig. 3.5c). The centre of the second peak corresponds to the average fluorescence intensity of single pHI molecules. To compare it with the neuronal single vesicle measurements I repeated the photobleaching experiment at a physiological pH of 7.4 (Fig. 3.5c). I observed a shift in the mean size to $15.3 \pm 0.34$ a.u., fully consistent with the described $\mathrm{pH}$-dependence of the superecliptic pHI fluorescence (Sankaranarayanan et al., 2000). Likewise, the distributions of dwell times before bleaching at $\mathrm{pH} 7.4$ and 9 , well described by single exponentials, reflected the same pH-dependence (Fig. 3.5d). By further extrapolation, one could assess the fraction of vesicular $\mathrm{pHI}$ molecules (at $\mathrm{pH} 5.5)$ bleached during a time period of $50 \mathrm{~s}$. Since there is $\sim 25$ fold fluorescence change of $\mathrm{pHI}$ molecules upon $\mathrm{pH}$ transition from 5.5 to 7.4 (Balaji and Ryan, 2007; Sankaranarayanan et al., 2000), this fraction amounted to less than $20 \%$ consistent with the bulk measurements (Fig. 3.2). Comparison of the single pHI fluorescence intensity with the quantal fluorescence amplitude suggested that SVs, on average, bear one $\mathrm{pHI}$ molecule $(0.97 \pm 0.02 \mathrm{SpH}, 0.99 \pm 0.02$ vGlut-pHI and $0.96 \pm 0.02 \mathrm{Syp}-\mathrm{pHI})$. Although the quantal size corresponds precisely to a single pHI molecule, the higher-order peaks in Fig. $\mathbf{3 . 4}$ could well indicate fusion of SVs with greater number of $\mathrm{pH}$ reporters or of multiple SVs. Overall these estimates are in good agreement with previous studies suggesting that the number of fusion proteins targeted per vesicle is low, close to one (Balaji and Ryan, 2007; Fernandez-Alfonso et al., 2006; Gandhi and Stevens, 2003). 
(a)

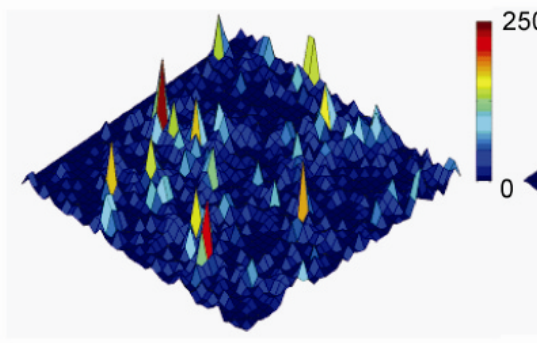

(c)

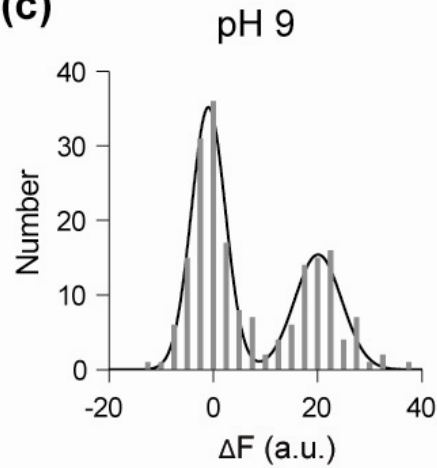

250

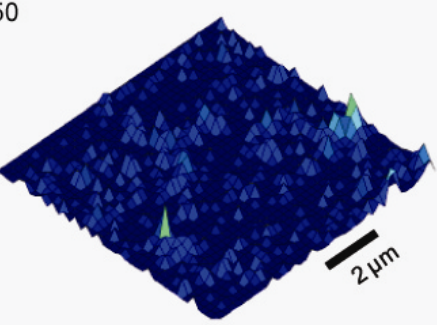

(b)

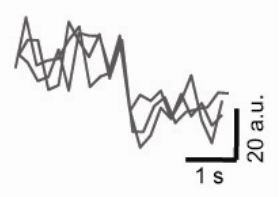

Figure 3.5: In vitro single molecule imaging reveals only few copies of pHI-tagged proteins are targeted to SVs.

(a) Surface plot of purified pHI molecules in a polyacrylamide gel before (left) and after (right) bleaching.

(b) Exemplar intensity traces (average of 3 data points) show instantaneous single molecule bleaching steps.

(c) Single molecule $\Delta \mathrm{F}$ distribution (binned at 2.5 a.u.). The solid line is the overall fit to multiple Gaussians of a mean unitary size $(\mathrm{m})$ of $20.11 \pm 1.09$ a.u. at $\mathrm{pH} 9\left(\mathrm{~N}=195\right.$, adjusted $\left.\mathrm{R}^{2}=0.94\right)$ and $15.06 \pm 0.35$ a.u. at $\mathrm{pH} 7.4\left(\mathrm{~N}=262\right.$, adjusted $\left.\mathrm{R}^{2}=0.98\right)$.

(d) Distributions of bleaching waiting times of $\mathrm{pH}$ at $\mathrm{pH} 9$ (black) and 7.4 (grey) are fitted to monoexponential functions (blue $-\mathrm{pH} 9$, red $-\mathrm{pH} 7.4$ ) yielding time constants of $7.35 \pm 0.43 \mathrm{~s}$ at $\mathrm{pH} 9$ and 9.65 $\pm 0.28 \mathrm{~s}$ at $\mathrm{pH} 7.4$. 


\subsection{3b Single molecule bleaching analysis of pHluorin in neuronal measurements}

The above single SV recordings in hippocampal boutons have the resolution of single SpH molecules. Therefore quite often, distinct bleaching steps of fluorescent $\mathrm{SpH}$ at the presynaptic membrane are observed randomly during the time course of the optical recordings $(0.5 \mathrm{~s}$ at $100 \mathrm{~Hz})$ (Fig. 3.6). Since these bleaching steps are not time-locked to the stimulus (Fig. 3.6), their occurrence at any of the frames before or after stimulation, which are used to calculate the fluorescence amplitude, can smear the noise peak in the $\Delta \mathrm{F}$ distributions towards the negative values. Therefore in the noise peak of the distributions in Fig. 3.3, even peaks or shoulders can be discerned corresponding to -1 or $-2 \mathrm{pH}$ molecules. On the contrary, the noise peak in the immobilized pHI calibration experiments (Fig. 3.5) does not suffer from this drawback (where locking in time is done with respect to a step-like bleaching event, not with respect to a stimulus) and therefore is a more reliable indicator of the actual baseline variability in these measurements, since the optical settings for both the gel and neuronal measurements were identical. The bleaching steps were thus quantified to get a direct correlate of single $\mathrm{pHI}$ molecule fluorescence intensity in an identical fluorophore environment (Fig. 3.6). The $\Delta \mathrm{F}$ distribution of bleaching steps was well described by a single Gaussian curve with a mean size of $14.1 \pm 0.19$ a.u. (Fig. 3.6) identical to the single pHI molecule intensity calculation (Fig. 3.6). Therefore, both the immobilized gel and neuronal measurements of bleaching steps provide similar and hence reliable estimates of single $\mathrm{pHI}$ molecule intensity. 
(a)

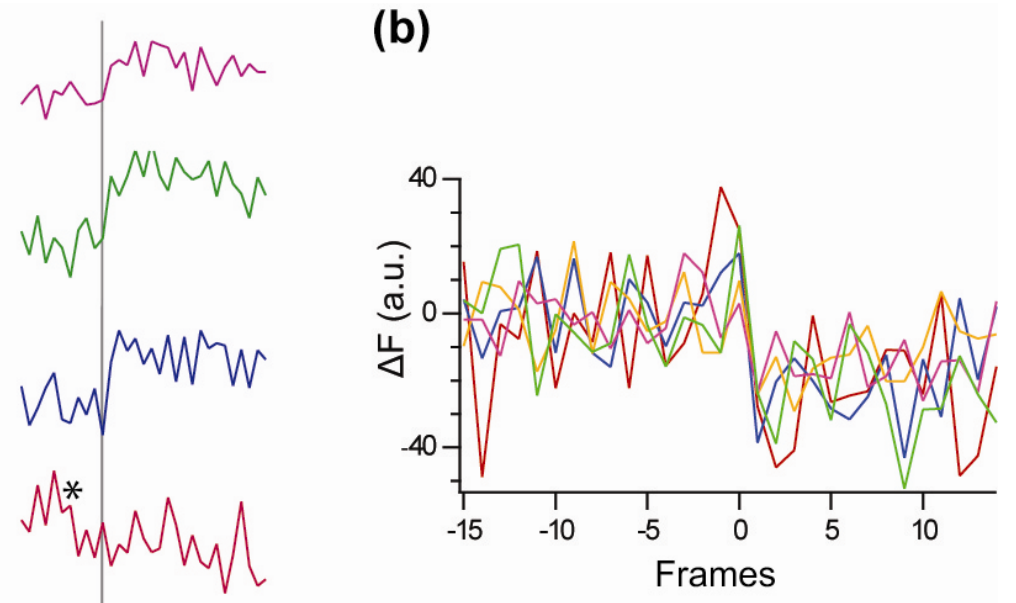

(c)

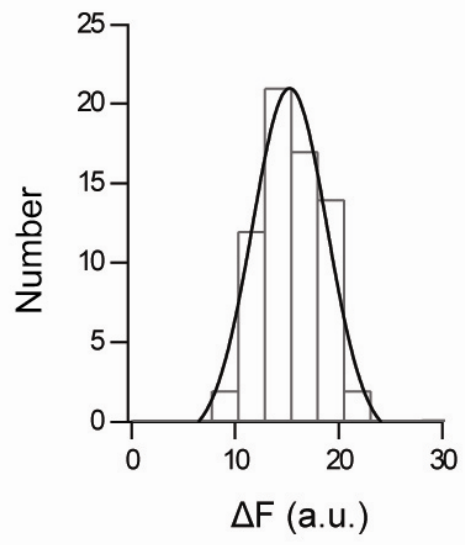

Figure 3.6: Single molecule fluorescence intensity quantified from bleaching steps during neuronal measurements.

(a) Exemplar raw fluorescence traces indicating photobleaching steps occurring randomly (denoted by *) during the single vesicle optical recordings not locked to the stimulus (grey bar) as opposed to the top 3 evoked responses.

(b) Photobleaching steps centered at the point of bleaching to quantitate the step-size and hence single molecule intensity.

(c) Amplitude distribution (bin width 2.5 a.u.) of the fluorescence downsteps $(n=102)$ is well fit with a Gaussian curve denoted by the solid line. The unitary size estimated was $15.2 \pm 0.29$ a.u. 


\subsubsection{Average number of pHluorin reporters targeted per synaptic vesicle}

Although the quantal size corresponds precisely to a single $\mathrm{pHI}$ molecule, the higher-order peaks in Fig. 3.4 could represent multiple fusion events or fusion of SVs with greater number of $\mathrm{pHI}$ reporters. Therefore to quantify the average number of $\mathrm{pHI}$ reporters per $\mathrm{SV}$, the single AP $\Delta$ F distributions (Fig. 3.4) were binned with increasing binwidth till the distribution could no longer be fit to a multiple Gaussian. Increasing the bin-width four-fold resulted in a quantal distribution, which was well described with a multiple Gaussian function. The quantal size estimated was very similar for the different reporters, namely $46.8 \pm 1.44$ a.u. for Syp-pHI, 46.5 \pm 1.95 a.u. for vGlut-pHI, and $35.6 \pm 3.85$ a.u. for $\mathrm{SpH}$ (Fig. S2). Comparison of the single pHI fluorescence intensity (Fig. 3.5) with the quantal fluorescence amplitude suggested that SVs bear on average $2.3 \pm 0.25 \mathrm{SpH}, 3.0 \pm 0.14$ vGlut-pHI, and $3.1 \pm 0.11$ Syp-pHI molecules (Fig. S2). The one-molecule peak previously distinct in Fig. 3.4 merged into the noise peak resulting in a large zero-order peak and the two and three molecule peak merged into the first non-zero peak (Fig. S2). Any further increase in binwidth caused a smearing with a positive tail, which could no longer be fit with a multi Gaussian function (Fig. S2). Overall these estimates are consistent with previous studies suggesting that the number of fusion proteins targeted per SV is low (Balaji and Ryan, 2007; Fernandez-Alfonso et al., 2006; Gandhi and Stevens, 2003) and there is a sizeable fraction of SVs with one pHI-tagged reporter. 


\subsubsection{SynaptopHluorin rescues $\mathrm{Ca}^{2+}$-evoked release in Syb2/Ceb null hippocampal neurons}

The single-vesicle intensity distribution with equidistant peaks at widths, close to the single $\mathrm{pHI}$ molecule fluorescence, revealed a sizeable fraction of SVs fusing with only a single copy of fluorescent $\mathrm{SpH}$. Note, that in WT boutons overexpressing $\mathrm{SpH}$, these SVs could contain in addition the endogenous complement of up to 70 non-fluorescent Syb2 (Takamori et al., 2006), which might participate in SNARE complex formation. Thus, it is not possible in this system to estimate the number of Syb2 molecules required for exocytosis from measuring copy numbers of $\mathrm{SpH}$. To overcome this problem, I expressed $\mathrm{SpH}$ in hippocampal neurons isolated from Syb2/Ceb DKO mice, thus creating a situation in which neurons contained $\mathrm{SpH}$ as the only Syb2 variant. In the DKO mice, secretion is completely impaired from adrenal chromaffin cells (Borisovska et al., 2005). Furthermore, deletion of Syb2 alone resulted in a 10-fold reduction of spontaneous fusion and 100-fold decrease in fast $\mathrm{Ca}^{2+}$-triggered exocytosis in hippocampal synapses (Schoch et al., 2001), thus providing a clean loss-offunction background for rescue experiments. Indeed, expression of Syb2 fused to fluorescent proteins at the lumenal domain in KO neurons was previously shown to rescue evoked exocytosis (Deak et al., 2006; Guzman et al., 2010). Hence the ability of SpH to rescue synaptic function in Syb2 KO hippocampal synapses was first tested (Fig. 3.7). First, the size of the recycling pool of SVs was estimated using the alkaline trap method (Guzman et al., 2010; Sankaranarayanan and Ryan, 2001). In this method, the vesicle proton pump, vATPase, that reacidifies the lumen of SV upon endocytosis, was blocked by a specific inhibitor folimycin, which arrested the SVs in an alkaline state after fusion. Once the entire recycling pool $\left(R_{c}\right)$ was depleted with a train of 900 APs at $20 \mathrm{~Hz}$ in the presence of folimycin, the fluorescence rose and plateaud with no recovery due to reacidification (Fig. 3.7a). This increase in fluorescence signified a pure exocytic signal. Neurons were then superfused with $\mathrm{NH}_{4} \mathrm{Cl}$ solution immediately post-stimulation (Fig. $3.7 \mathrm{a} \&$ b), thereby neutralizing the $\mathrm{pH}$ across all membranes to 7.4 and thereby unmasking any SVs that previously did not fuse. The fluorescence increase in response to the depleting stimulation relative to the total increase (T) in response to the $\mathrm{NH}_{4} \mathrm{Cl}$ pulse then yielded the fractional size of the recycling pool $\left(R_{c} / T\right)$. 
Fluorescence responses from the DKO neurons were indistinguishable from WT (Fig. 3.7b), as was the distribution of recycling pool fraction. The peak of the distribution was at $\sim 0.45$, consistent with previous size estimates of recycling pool (Sankaranarayanan and Ryan, 2001) (Fig. 3.7c). The above experiment was repeated with a train of $900 \mathrm{APs}$ at $5 \mathrm{~Hz}$, in hippocampal synapses, to look at SV repriming and re-release over prolonged stimulation in hippocampal synapses. After stimulation the residual fluorescence was dequenched with a $\mathrm{NH}_{4} \mathrm{Cl}$ pulse. Average traces from DKO boutons seemed identical to WT both in terms of kinetics and relative amplitude (Fig. 3.7d). Amplitude histogram of the fractional increase in fluorescence upon stimulation (a) to the total increase after $\mathrm{NH}_{4} \mathrm{Cl}$ (b) perfectly overlapped between the DKO and WT boutons (Fig. 3.7e) indicating that $\mathrm{SpH}$ could fully rescue evoked release in the DKO neurons. 
(a)

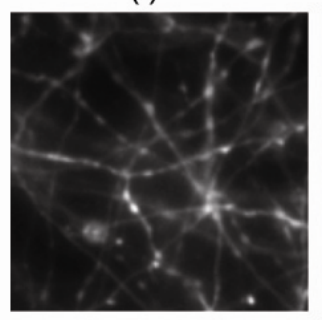

(b)

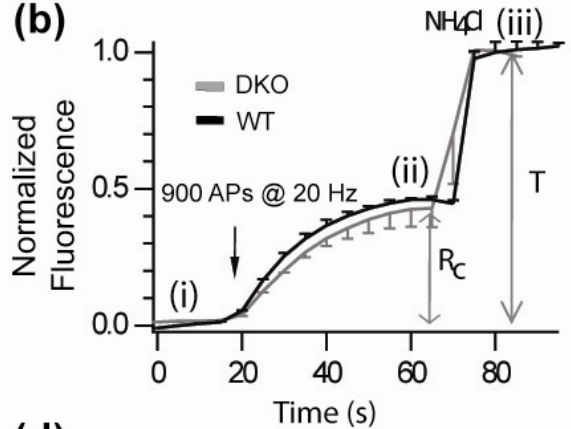

(d)

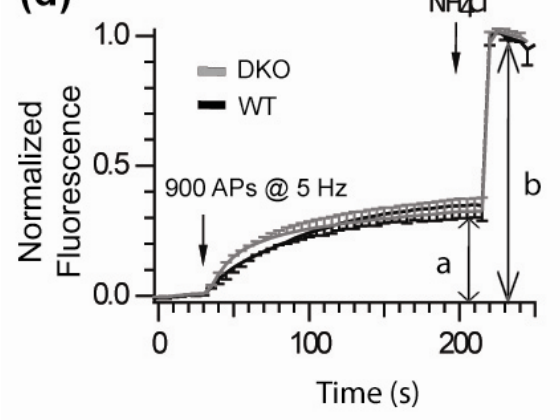

(ii)

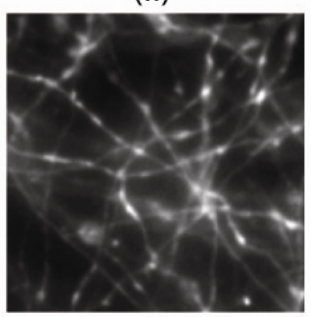

(iii)

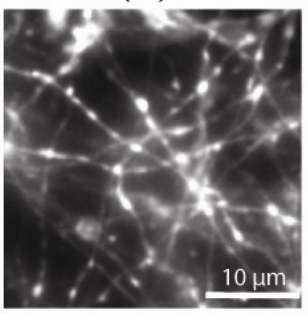

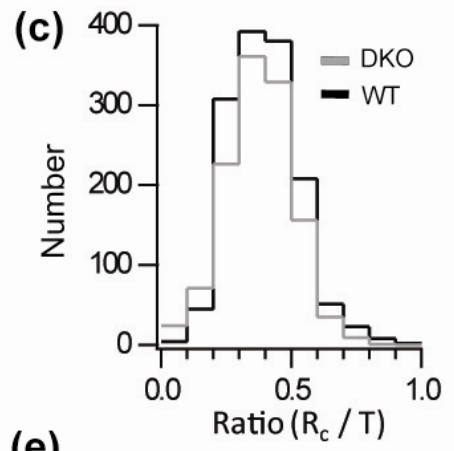

(e)

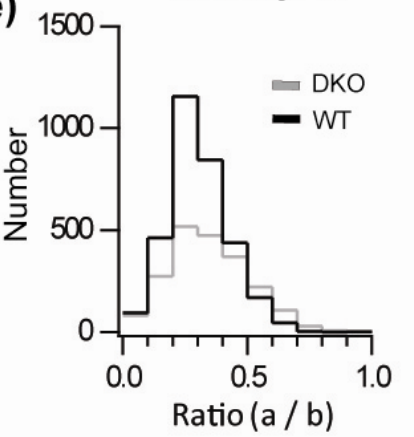

Figure 3.7: SpH overexpression in Syb2/Ceb DKO neurons rescues evoked synaptic transmission.

(a) Fluorescence images of DKO hippocampal boutons overexpressing SpH before (i) and after (ii) 900 APs stimulation followed by $\mathrm{NH}_{4} \mathrm{Cl}$ application (iii).

(b) Exemplar average fluorescence responses (DKO in gray and WT in black; $\mathrm{n}>300$ boutons from 2 experiments for both conditions) to $900 \mathrm{APs}$ at $20 \mathrm{~Hz}$ followed by $\mathrm{NH}_{4} \mathrm{Cl}$ dequenching. The relative increase in fluorescence upon stimulation in the presence of folimycin denotes the recycling pool size $\left(R_{c}\right)$. Subsequent fluorescence increase upon $\mathrm{NH}_{4} \mathrm{Cl}$ application denotes the size of the total pool $(T)$.

(c) $\Delta \mathrm{F}$ distributions of the recycling pool fraction from $\mathrm{SpH}$-overexpressing $\mathrm{DKO}$ (gray; $\mathrm{n}>1000$ boutons from 6 experiments) and WT (black; $n>800$ boutons from 4 experiments) boutons are similar .

(d) Exemplar average fluorescence response (DKO in gray and WT in black; $\mathrm{n}>300$ boutons from 2 experiments for both conditions) to $900 \mathrm{APs}$ at $5 \mathrm{~Hz}$ (a) followed by $\mathrm{NH}_{4} \mathrm{Cl}$ dequenching (b).

(e) $\Delta \mathrm{F}$ distributions of the relative increase in fluorescence upon stimulation (a / b) from DKO (gray ; $\mathrm{n}>$ 1500 boutons from 7 experiments) and WT (black; $n>1200$ boutons from 6 experiments) boutons are similar. 
The above results indicated that the fusion construct $\mathrm{SpH}$ is functional and fully restored stimulation dependent exocytosis in the DKO neurons, in agreement with previous electrophysiology measurements of Syb2 KO hippocampal neurons expressing $\mathrm{C}$ - and $\mathrm{N}$ terminal GFP fusion constructs of Syb2 (Deak et al., 2006). I further quantified the fluorescence responses to weaker stimuli such as trains of $40 \mathrm{APs}$ at $20 \mathrm{~Hz}$, a protocol that selectively depletes the RRP, or $100 \mathrm{APs}$, in the absence of folimycin. SpH fluorescence profiles obtained from DKO neurons were indistinguishable from WT (Fig. 3.8a), as well as their amplitude distributions (Fig. 3.8b).

(a)

$$
100 \text { APs@ } 20 \mathrm{~Hz}
$$
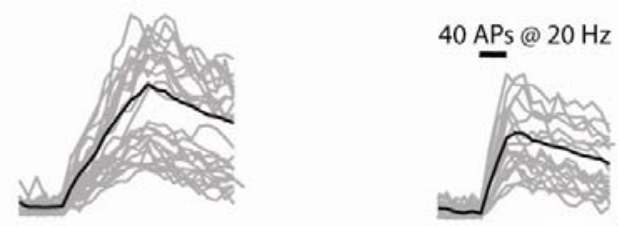

(b)
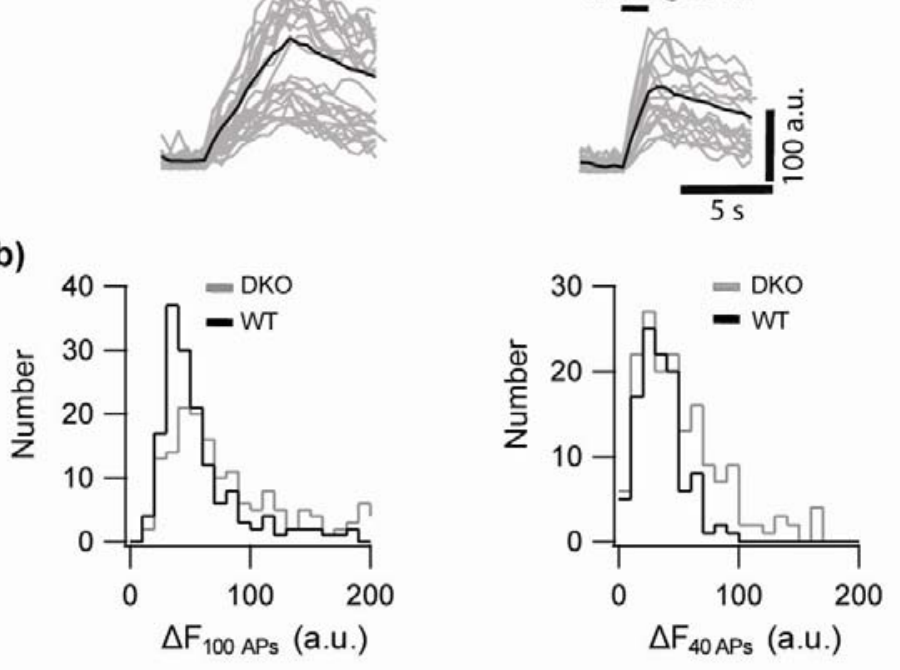

Figure 3.8: Fluorescence responses to 40 and $100 \mathrm{APs}$ are unaltered in SpH-overexpressing DKO boutons.

(a) Fluorescence responses (gray traces) of individual boutons to 100 APs (left) and 40 APs (right) at 20 $\mathrm{Hz}$. The average response is overlaid (black line).

(b) $\Delta \mathrm{F}$ distributions from $\mathrm{SpH}$-overexpressing $\mathrm{DKO}$ (gray; $\mathrm{n}>150$ boutons from 6 experiments) and WT (black; $n>150$ boutons from 6 experiments) boutons in response to 100 APs (left) and 40 APs (right) were similar. 


\subsubsection{Two copies of SynaptopHluorin are necessary and sufficient for evoked SV fusion}

In simple terms the above amplitude distributions reflect the product of $\mathrm{P}_{\mathrm{r}}$ during stimulation and the quantal content, i.e. the number of $\mathrm{SpH}$ molecules per SV. Thus, a similar distribution might either reflect a low $\mathrm{P}_{\mathrm{r}}$, i.e. incomplete rescue by $\mathrm{SpH}$, combined with a high $\mathrm{SpH}$ copy number per fusing SV (high quantal content), or a full rescue (same $P_{r}$ ) with the same low copy number of about one SpH molecule per SV as in WT (Fig. 3.9). The latter case would imply that only one copy of $\mathrm{SpH}$, i.e. capable of forming a single SNARE complex, can rescue rapid exocytosis in the DKO neurons. To resolve between these two scenarios, single AP evoked $\mathrm{SpH}$ fluorescence responses were measured similar to those in the WT neurons. The resulting amplitude histogram looked strikingly similar to the WT distribution with a peak spacing of $14.9 \pm 0.28$ a.u. except for the first non-zero peak (Fig. 3.9b). Superposition of both the quantal histograms revealed an almost complete lack of the one molecule peak in the DKO rescue histogram (Fig. 3.9c) suggesting that SVs with only one copy of $\mathrm{SpH}$ are unable to fuse within ms upon stimulation. However, the higher order peaks from the DKO rescue and WT histograms were perfectly aligned to one another. The prominence of the single molecule peak in the WT histogram and its absence from the DKO rescue histogram signifies that minimally two molecules of $\mathrm{SpH}$ are required to promote rapid vesicle fusion within less than $10 \mathrm{~ms}$ after stimulation, i.e. within one frame, at our $100 \mathrm{~Hz}$ sampling frequency. Thus, two copies of SpH have to engage in SNARE pairing, and these two SNARE complexes are necessary and sufficient for SV fusion during fast synaptic transmission. 
(a)
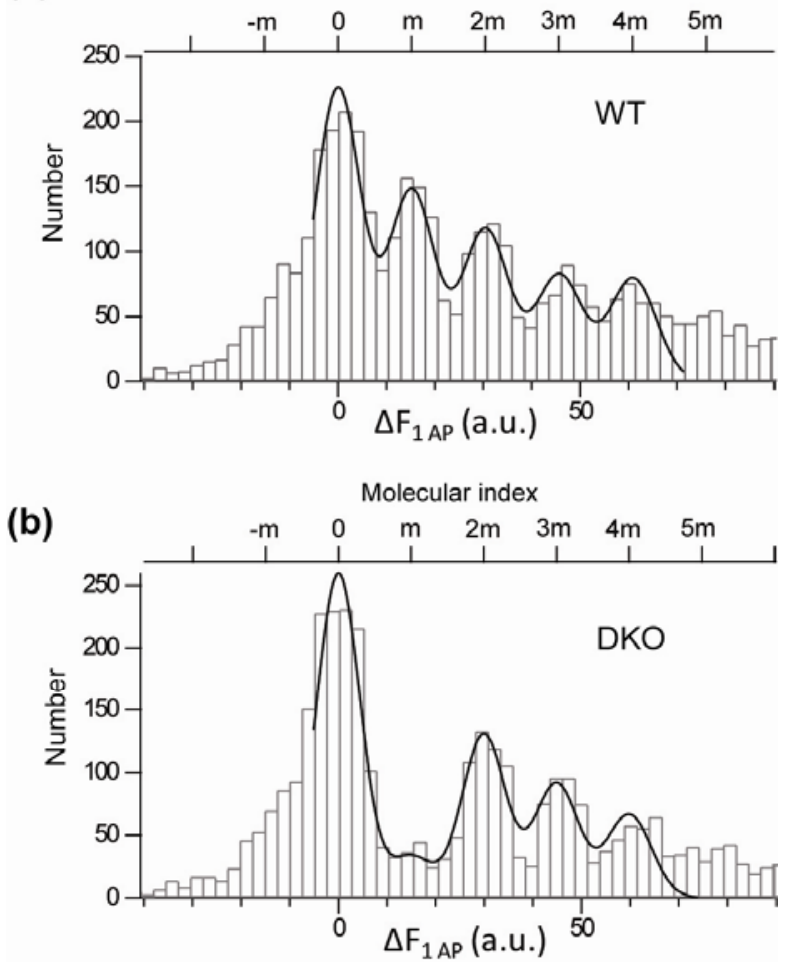

(c)

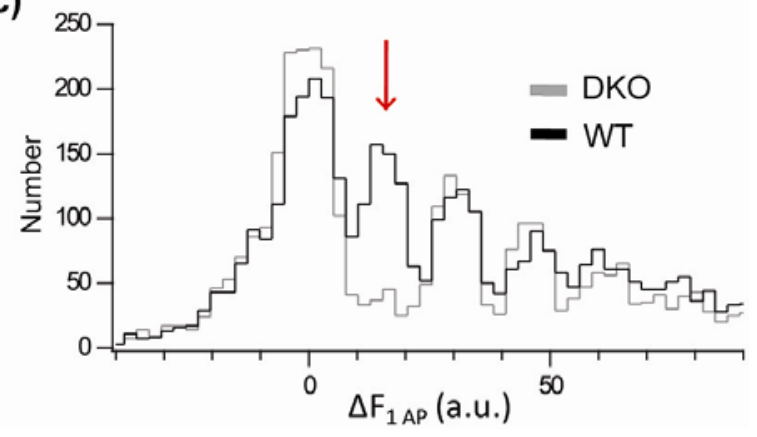

Figure 3.9: Two copies of SpH are minimally required to drive SV fusion.

(a) $\Delta \mathrm{F}$ distribution from $\mathrm{SpH}$-overexpressing WT boutons plotted with a bin width of 2.5 a.u. The smooth curve is the overall fit to multiple Gaussians (adjusted $R^{2}=0.94$ ). The estimated unitary size is $15.2 \pm$ 0.21 a.u. ( $\mathrm{n}>400$ boutons from 14 experiments).

(b) $\Delta \mathrm{F}$ distribution from $\mathrm{SpH}$-overexpressing $\mathrm{DKO}$ boutons plotted with a bin width of 2.5 a.u. The smooth curve is the overall fit to multiple Gaussians (adjusted $R^{2}=0.94$ ). The estimated unitary size is $14.9 \pm 0.28$ a.u. ( $n>400$ boutons from 15 experiments).

(c) Superimposed intensity distributions for SpH-overexpressing WT (gray) and DKO boutons (black). The red arrow indicates the marked reduction of the first non-zero peak in spH-overexpressing DKO boutons. 


\subsubsection{Single SynaptopHluorin molecule is unable to drive evoked fusion}

In the single AP histograms of DKO rescue neurons, a small residual single molecule peak was evident, when comparing the absolute frequencies of the fusion events of SVs bearing one, two or three SpH molecules (Fig. 3.10a). This indicates that SVs with one molecule of $\mathrm{SpH}$ may occasionally fuse. However, it cannot be excluded that during the prebleaching period a small fraction of $\mathrm{SpH}$ inside the SV may become bleached despite the low $\mathrm{pH}$ and will go undetected, thus leading to an underestimation of the Syb2 copy number. To resolve this issue, the duration of photobleaching was increased to more than two-fold i.e. 2 min (Fig. 3.10b). Since all the non-zero peaks of the histograms are affected at the same rate the resulting ratio is more or less constant for WT neurons (Fig. 3.10c). The relative ratio of the amplitudes of the first over the second non-zero peak was quantified. Indeed, the ratio more than doubled when the prebleaching time was increased for DKO rescue neurons (Fig. 3.10d). Thus, the small one molecule peak observed in the histogram of DKO boutons is rather a consequence of prebleaching than an indication of SV fusion with only one SNARE complex.

Due to irreversible prebleaching of $\sim 20 \%$ of the vesicle resident $\mathrm{SpH}$, local recycling of the rapidly releasable vesicles containing the bleached $\mathrm{SpH}$ might underestimate the actual number of SpH molecules per SV during the later half of the recording period. On comparing the intensity distributions from the first five consecutive recording periods to the next five for WT and DKO rescue boutons there was no significant change in the relative frequency of the one to two molecule peak (Fig. 3.10e \& f), indicating little or no local recycling of SVs containing bleached $\mathrm{SpH}$ within the measurement period. 
(a)

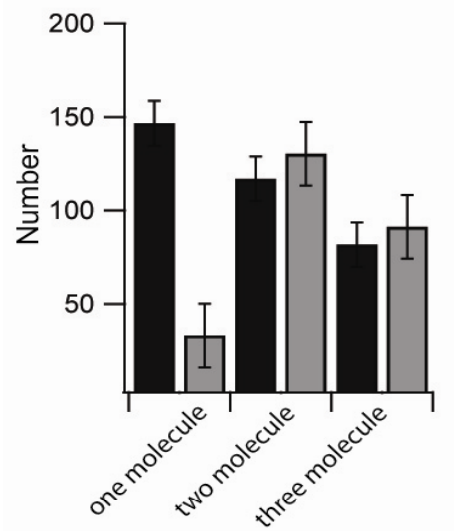

(d)

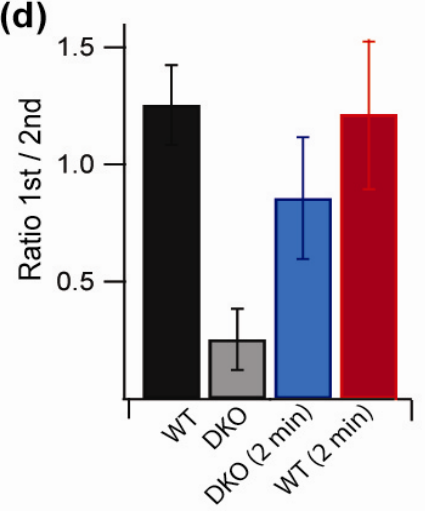

(b)

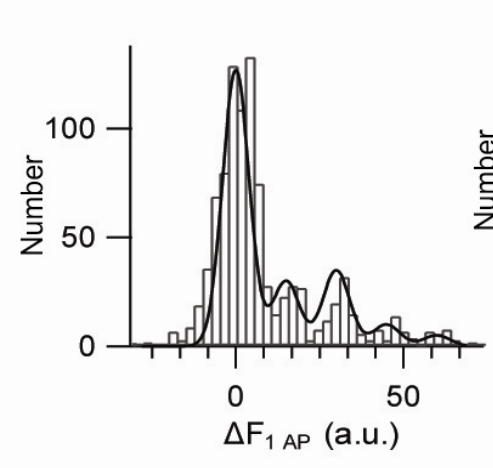

(e)

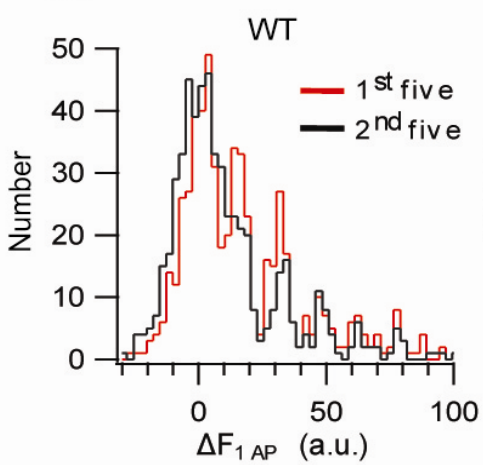

(c)

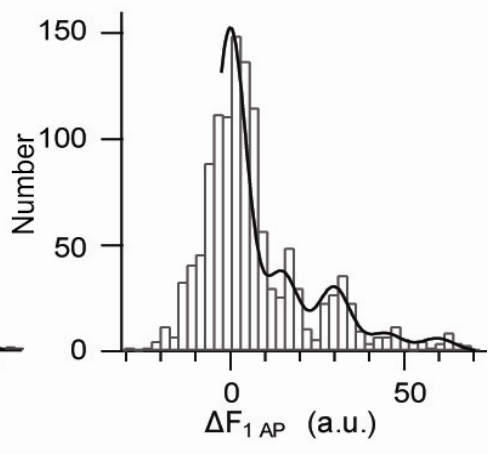

(f)

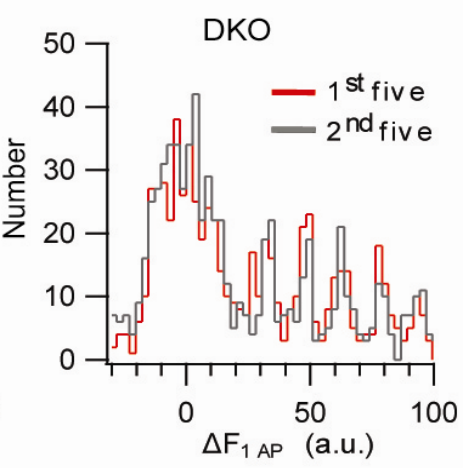

Figure 3.10: SVs with one copy of $\mathrm{SpH}$ are unable to fuse.

(a) Bar graph comparing the absolute amplitudes of the non-zero peaks from $\mathrm{SpH} \triangle \mathrm{F}$ histograms for WT (black) and DKO (grey) boutons. The amplitudes were obtained from the best-fit model (error bars represent s.d).

(b) $\Delta \mathrm{F}$ histogram after $2 \mathrm{~min}$ of prebleaching in $\mathrm{SpH}$-expressing $\mathrm{DKO}$ boutons shows 'recovery' of the first non-zero peak. Superimposed is the best-fit Gaussian curve (adjusted $R^{2}=0.89$ ) with a unitary size of $15.0 \pm 0.50$ a.u. ( $n>100$ boutons from 5 experiments).

(c) $\Delta \mathrm{F}$ histogram after $2 \mathrm{~min}$ of prebleaching in $\mathrm{SpH}$-expressing $\mathrm{WT}$ boutons. Superimposed is the bestfit Gaussian distribution (adjusted $R^{2}=0.94$ ). The unitary size estimated was $14.9 \pm 0.67$.

(d) Bar diagram showing relative amplitudes expressed as the ratio of the first non-zero peak to the second non-zero peak for WT (black), DKO (gray), and boutons prebleached for 2 min from DKO (blue) and WT (red) neurons (error bars represent s.d.).

(e \& f) The $\Delta \mathrm{F}$ distributions from SpH-expressing WT (e) and DKO boutons (f) of the first five trials of the experiments were compared to the last five. Bin width is 2.5 a.u. The peaks overlap and the relative amplitudes of the first to the second non-zero peaks are similar in both, WT and DKO histograms. 
Does the missing one molecule peak have any effect on the net amplitude distribution? To address this, intensity-weighted histograms were generated. A slight difference of $\sim 10 \%$ was observed for DKO neurons compared to WT, better seen in the integrated distribution, which implies that reduction of the single molecule peak does not alter the net shape of the distribution. Hence, total release was unperturbed even when SVs with a single SpH copy are unable to fuse. This finding is in agreement with the amplitude distributions of 40,100 and 900 APs (Fig. 3.7 and Fig. 3.8).

(a)

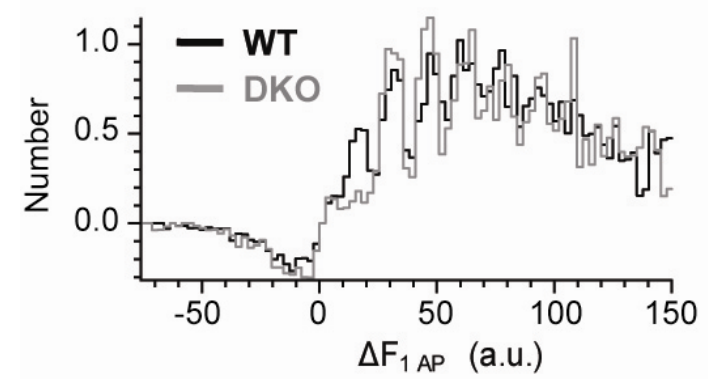

(b)

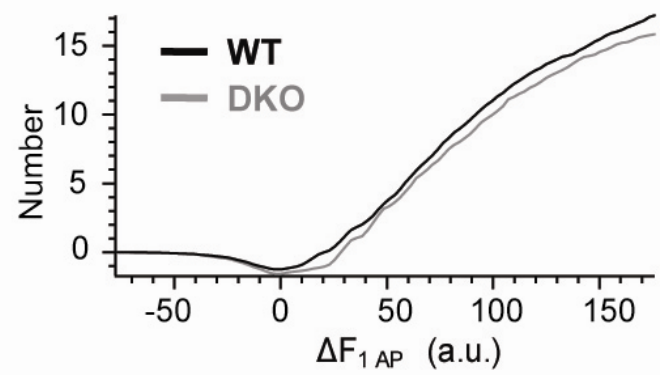

Figure 3.11: Effect of missing one molecule peak in DKO histograms on net fluorescence amplitude.

(a) Intensity-weighted histograms generated by multiplying each bin with its respective amplitude. The histogram was then normalized to the total number of events. The total sum of the histogram was then calculated i.e. WT $=34.41$ a.u. and DKO = 31.69 a.u. A slight but negligible difference $(<10 \%)$ between the WT and DKO histograms was observed.

(b) This difference is better visible in the integrated histograms. Thus, the missing one molecule peak does not affect the net fluorescence amplitude. 


\subsubsection{SynaptopHluorin is not proteolytically cleaved}

Underestimation of the actual copy number of $\mathrm{SpH}$ required to evoke fusion may result from the expression of $\mathrm{SpH}$ molecules that are non-fluorescent, either due to misfolding or by posttranslational proteolytic cleavage of the GFP-moiety. Misfolding of GFP moieties was previously estimated to account for maximally 10-20\%(Ulbrich and Isacoff, 2007) i.e. too low to account for a systematic underestimation of the number of $\mathrm{pHI}$ molecules or SNARE complexes. Moreover, the low number of $\mathrm{SpH}$ found to be sufficient to fuse SVs raises the concern that many $\mathrm{pH}$ moieties might be cleaved from $\mathrm{SpH}$ during trafficking to the SVs, resulting in copies of unlabeled Syb2 in SVs, which would go undetected in my measurements. To address this, in collaboration with Dr. Saheeb Ahmed from the laboratory of Prof. Reinhard Jahn (Dept. of Neurobiology; Max-Planck Inst for Biophysical Chemistry), western blot analysis of lysates from DKO hippocampal cultures overexpressing $\mathrm{SpH}$ was performed with an anti-Syb2 antibody that specifically binds to the $\mathrm{N}$-terminus (aa 2 - 17) of Syb2 (note that $\mathrm{pHI}$ is fused to the luminal C-terminus and thus does not interfere with detection) (Fig. 3.12). The presence of a single SpH band with no cleaved Syb2 product confirmed that there is no significant cleavage of the fusion protein, $\mathrm{SpH}$, when overexpressed on the DKO background. In contrast, two separate bands, one correlating to endogenous Syb2 and one representative of $\mathrm{SpH}$ could be detected from lysates of WT cells overexpressing SpH (Fig 3.12). 


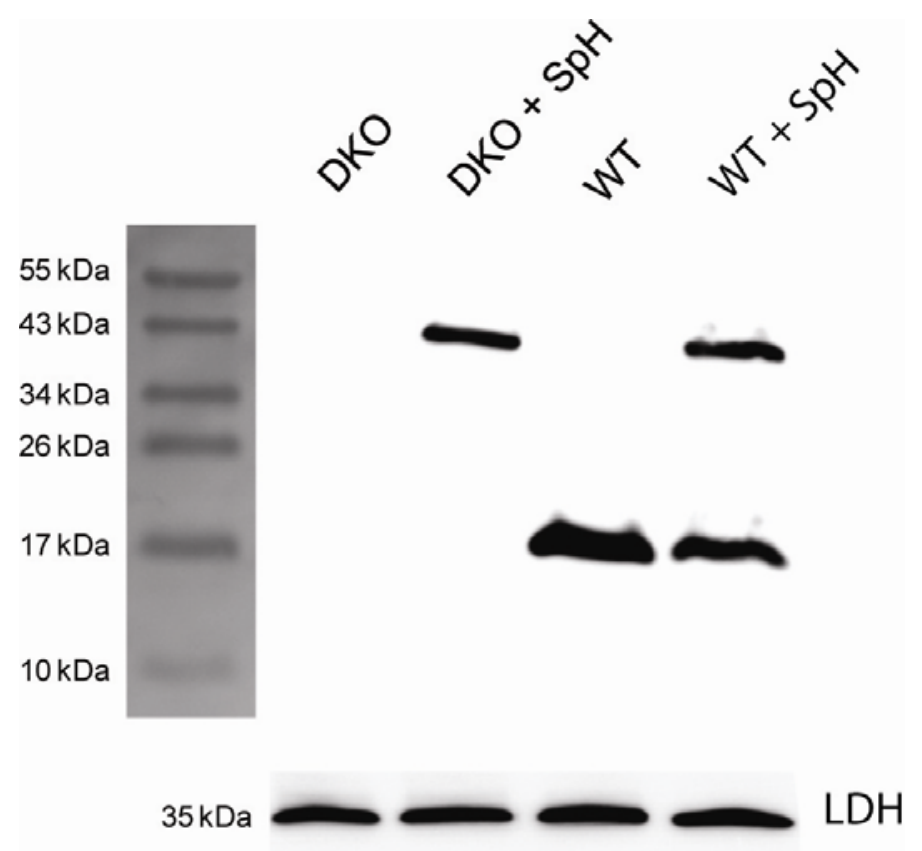

Figure 3.12: No cleavage of pHI upon SpH overexpression in DKO neurons.

Immunoblot of lysates from WT, DKO and SpH-overexpressing DKO and WT neuronal cultures probed with anti-Syb2 antibody (mouse monoclonal, 69.1). Endogenous Syb2 runs at $\sim 16 \mathrm{kDa}$, while Syb2 fused to $\mathrm{pH}$ should be shifted by $\sim 25 \mathrm{kDa}$. Thus, the single band at $\sim 40 \mathrm{kDa}$, in $\mathrm{DKO}+\mathrm{SpH}$ lane, corresponds to uncleaved $\mathrm{SpH}$. Note the absence of any cleaved Syb2 product at $\sim 16 \mathrm{kDa}$ in the $\mathrm{DKO}+\mathrm{SpH}$ lane. The lysates from WT cells show a single 16kDa endogenous Syb2 band, while WT cells overexpressing $\mathrm{SpH}$ have an additional $\mathrm{SpH}$ band at $\sim 40 \mathrm{kDa}$. The loading control used was $\mathrm{LDH}$ and shows nearly equal loading of the samples.

In summary, the above data provide the first evidence that two molecules of $\mathrm{SpH}$ and thus two SNARE complexes are necessary and sufficient for triggered SV fusion in living central synapses. 


\subsection{Number of Synaptotagmin1 molecules required to trigger fusion}

By optically recording single vesicle fusion with $\mathrm{pHI}$-fusion chimeras of different SV proteins followed by single $\mathrm{pHI}$ molecule calibration, it is possible to precisely quantify their copy numbers per SV. Therefore, I tested this approach on another interesting SV protein crucial for evoked fusion, namely Syt1. First, I estimated the number of Syt1-pHI molecules per SV on a WT background in the presence of the endogenous Syt1. Single APs were elicited in Syt1-pHI transfected boutons to measure fusion of single SVs. Since the surface pool of Syt1-pHI is comparable to $\mathrm{SpH}$, fluorescence transients were easily resolvable by prebleaching Syt1-pHI transfected boutons for $50 \mathrm{~s}$, like in $\mathrm{SpH}$ overexpression (Fig.3.3). The fluorescence responses exhibited instantaneous increase upon stimulation similar to responses obtained from boutons expressing SpH, Syp-pHI or vGlut-pHI (Fig. 3.13, for comparison see Fig. 3.1 and 3.3). The $\Delta \mathrm{F}$ distribution consisted of several evenly spaced peaks. The quantal size determined by fitting the histogram to a multiple Gaussian function was $13.02 \pm 0.49$ a.u (Fig. 3.13). When compared with the single $\mathrm{pHI}$ fluorescence, it was estimated that SVs contain on an average $0.85 \pm 0.22 \mathrm{Syt} 1-\mathrm{pHI}$ molecules. This was in good agreement with other $\mathrm{pHI}$-based reporters, measured earlier, indicating that the sorting process of SV proteins is random with a considerable fraction of SVs bearing on an average single Syt1-pHI molecule. 
(a)
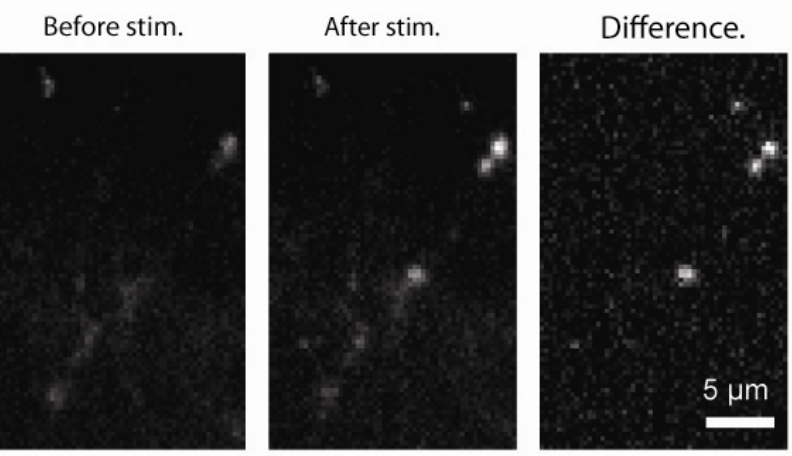

(b)

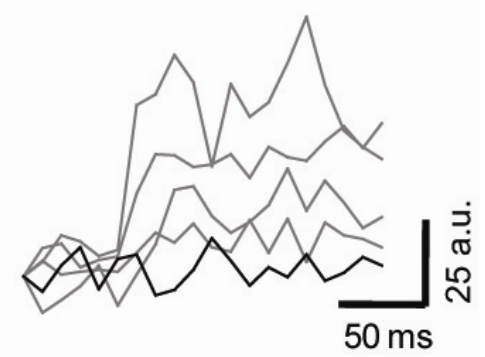

(c)

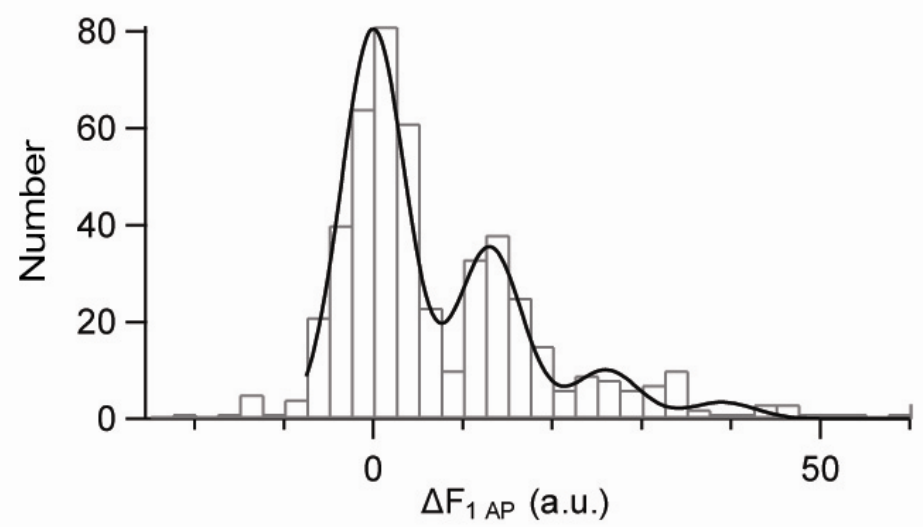

Figure 3.13: One Syt1-pHI molecule is targeted per SV when overexpressed on a WT background.

(a) Exemplar image of Syt1-pHI transfected boutons before and after 1 AP stimulation (Stim.). Difference image reveals sites of evoked release.

(b) Fluorescence transients from individual boutons (gray denotes responses and black denotes failures).

(c) $\Delta \mathrm{F}$ distribution of Syt1-pHI overexpressing boutons evoked by single APs plotted with a binwidth of 2.5 a.u ( $n>40$ boutons from 3 experiments). The smooth solid curve is the best overall fit to multiple Gaussians of fixed quantal size $q \pm$ s.d. The estimated $q$ is $13.0 \pm 0.49$ a.u.; adjusted $R^{2}=0.97$ ).

By overexpressing Syt1-pHI in Syt1 KO hippocampal neurons, and hence creating a scenario where Syt1-pHI is the only Syt1 variant, the effective copy number of Syt1 (required per SV) to rescue synchronous release could be determined. At first, the ability of Syt1-pHI to rescue fusion was tested in response to strong stimulation that depletes the recycling pool. A depletion pulse of 900 APs at $20 \mathrm{~Hz}$ was applied to measure the size of the recycling pool in 
the presence of folimycin and the remaining non-releasable pool was then dequenched with a subsequent pulse of $\mathrm{NH}_{4} \mathrm{Cl}$. Fluorescence increase after the $900 \mathrm{APs}$ stimulation and the total increase after $\mathrm{NH}_{4} \mathrm{Cl}$ application was plotted (Fig 3.14 a \& b). Amplitude distributions of the Syt1-pHI responses from KO neurons were perfectly superimposable on the responses from WT neurons implying the Syt1-pHI can rescue evoked release (Fig 3.14 a \& b). Previous studies in Syt1 KO neurons have shown that fast synchronous release is markedly reduced, but the slow asynchronous release persists (Geppert et al., 1994). Thus, the effect on total release in absence of Syt1 is quite debatable and seems to be dependent on the type of neuronal cultures such as autaptic cultures and mass cultures (Liu et al., 2009; NicholsonTomishima and Ryan, 2004). Therefore, it remains to be tested if Syt-pHI can rescue the kinetics of release in the KO neurons, which is the predominant effect upon Syt1 deletion.

Next, single vesicle optical recordings were performed in Syt1 KO neurons overexpressing Syt1-pHI. This way the fluorescence contribution of single vesicle fusion would give a direct estimate of the functional number of Syt1 molecules minimally necessary for synchronized fusion. Boutons expressing Syt1-pHI in KO neurons displayed robust fluorescence increases upon a single AP indistinguishable from WT responses (Fig 3.14c). The amplitude of fluorescence change was then plotted. The single AP distribution was quantal and could be well described by a multiple Gaussian, with several equidistant peaks and a sizeable fraction of SVs containing one Syt1-pHI molecule (Fig 3.14c). This was strikingly similar to the distribution observed from WT boutons (Fig 3.13). The quantal size estimated from the DKO distribution is $14.429 \pm 0.192$ a.u. equivalent to the WT quantal size (Fig 3.14c). On comparison with the fluorescence intensity of single $\mathrm{pH}$ molecule, it seems that a single copy of Syt1-pHI per SV is sufficient to sense $\mathrm{Ca}^{2+}$ influx and trigger fusion. 
(a)

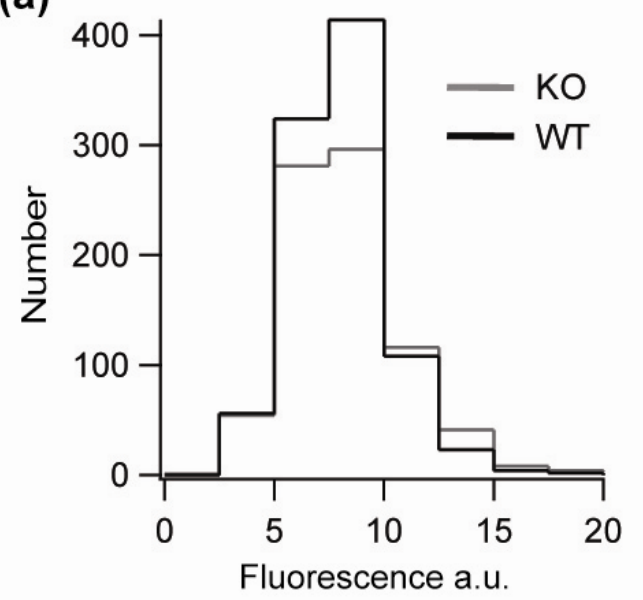

(c)

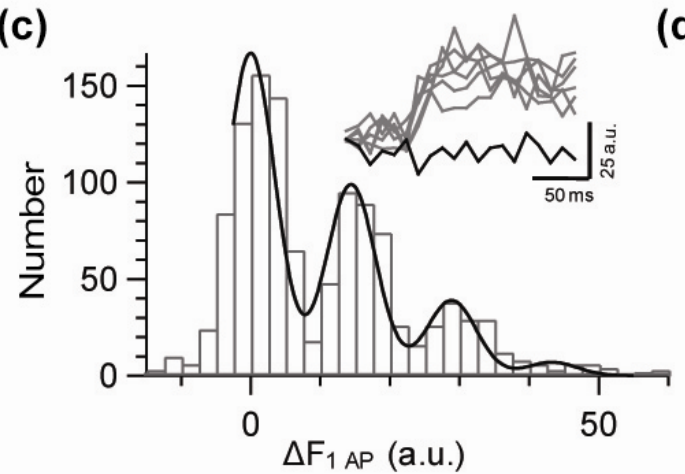

(b)

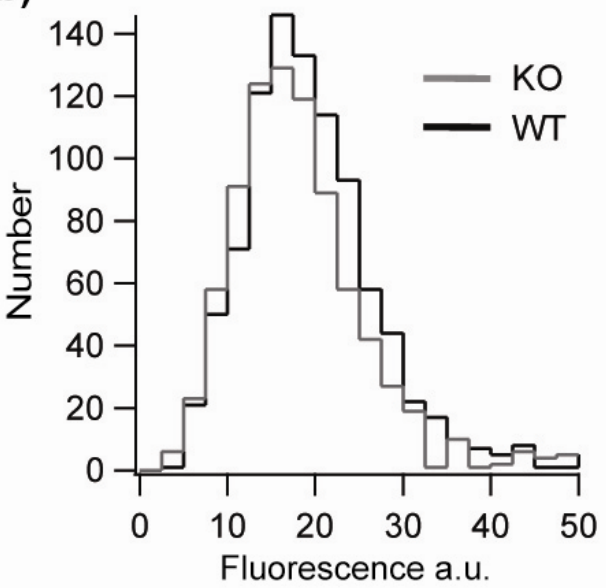

(d)

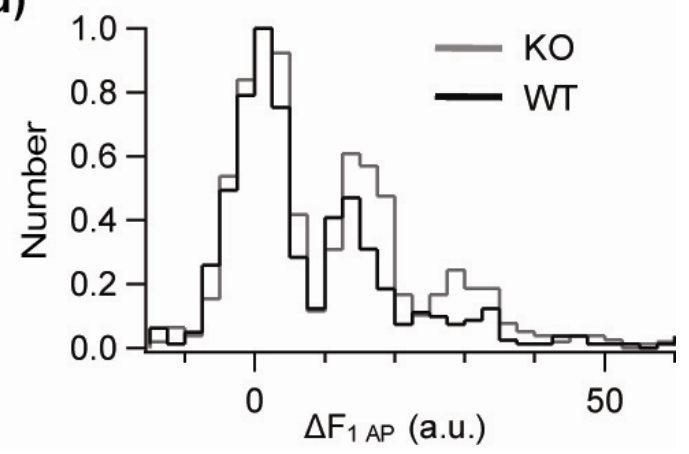

Figure 3.14: One Syt1-pHI molecule can apparently rescue fusion.

(a) $\Delta \mathrm{F}$ distributions of the recycling pool from Syt1-pHI-overexpressing KO (gray; $n>200$ boutons from 3 experiments) and WT (black ; $\mathrm{n} 200$ boutons from 3 experiments) boutons were similar.

(b) $\Delta \mathrm{F}$ distributions of the total SV pool from Syt1-pHI-overexpressing KO (gray ; $\mathrm{n}>200$ boutons from 3 experiments) and WT (black; $n>200$ boutons from 3 experiments) boutons overlapped perfectly.

(c) $\Delta \mathrm{F}$ distributions from Syt1-pHI overexpressing $\mathrm{KO}$ boutons evoked by 1 AP plotted with a binwidth of 2.5 a.u ( $n>100$ boutons from 6 experiments). The smooth solid curve is the overall best fit to multiple Gaussians of fixed quantal size $q \pm$ s.d. The estimated $q$ is $14.4 \pm 0.19$ a.u.(adjusted $R^{2}=0.92$ ). Inset shows exemplar fluorescence traces (gray denotes responses and black denotes failures).

(d) $\Delta \mathrm{F}_{1 \mathrm{AP}}$ histograms of Syt1-pHI transfected WT boutons overlaid on the responses from $\mathrm{KO}$ boutons displayed a perfect overlap indicating that one copy of Syt1-pHI is sufficient to initiate fusion. 


\subsection{Spatial and temporal kinetics of the readily retrievable pool of native vesicle proteins}

\subsection{1 $\mathrm{pH}-$ dependence of the cypHer probe}

To determine whether cypHer-coupled antibodies can reliably measure the $\mathrm{pH}$ changes during exo-endocytosis and hence map presynaptic activity, the $\mathrm{pH}$ dependence of its fluorescence properties was characterized. At first, hippocampal neurons were labeled with cypHer-coupled antibody against Syt1 (aSyt1-cypHer) by incubation at $37^{\circ} \mathrm{C}$ for 3-4 hrs (Material and Methods). Then neurons were fixed and permeabilized to access all the labeled Syt1 molecules. To calibrate the $\mathrm{pH}$-dependence of the cypHer dye, stained neurons were superfused with buffers of different $\mathrm{pH}$. At low $\mathrm{pH}(5.5)$ the fluorescence signal was maximal, while it reached a minimum at a $\mathrm{pH}$ of 9 (Fig. 3.15). The normalized fluorescence $(\Delta F / F)$ values were plotted as a function of $\mathrm{pH}$, which was then fitted to a simple HendersonHasselbalch equation (Material and methods; Fig. 3.15). The data indicate that the cypHer dye coupled to the antibody has a pK for protonation of 7.05 , ideal for measuring $\mathrm{pH}$ changes during exo- and endocytosis similar to the canonical GFP-based $\mathrm{pHI}$. Moreover, the $\mathrm{pH}-$ dependent fluorescence properties of the cypHer dye are exactly opposite to that of the GFPbased exo-endocytosis probe, $\mathrm{SpH}$ (Sankaranarayanan et al., 2000).

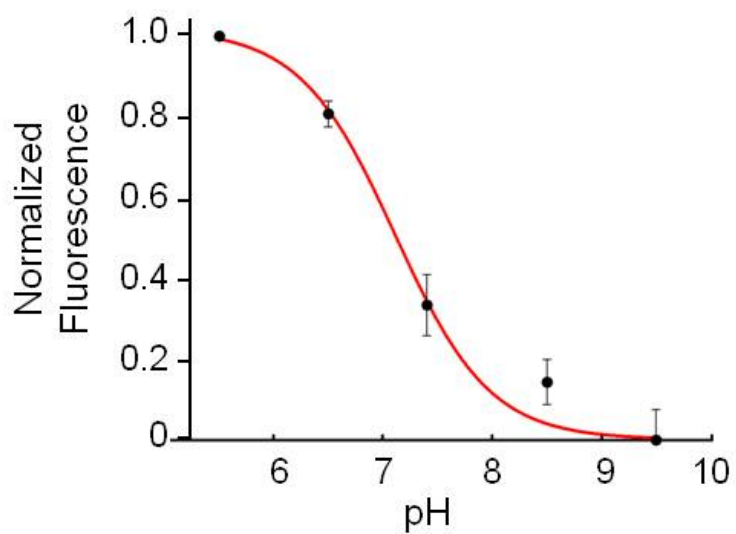

Figure 3.15: pH-titration curve of cypHer-based exogenous reporter.

The average normalized change in fluorescence of aSyt1-cypHer stained hippocampal boutons from multiple trials (each trial consisted of $>30$ boutons) as a function of $\mathrm{pH}$. The solid curve is a fit to a simple Henderson - Hasselbalch equation (adjusted $R^{2}=0.99$ ). The $\mathrm{pK}$ from the fit is 7.05 . 


\subsubsection{Live-cell labeling of neurons with cypHer-coupled antibodies}

In order to monitor SV recycling, live hippocampal neurons were labeled with cypHer-coupled antibodies, aSyt1-cypHer / aVGAT-cypHer, by incubating them at $37^{\circ} \mathrm{C}$ for $3-4$ hrs in the presence of $2 \mathrm{mM}$ external $\mathrm{Ca}^{2+}$ concentration to allow antibody uptake and labeling of the entire recycling pool (Material and Methods; Fig. 3.16a). These antibodies bind to their specific epitopes (Syt1 and VGAT respectively) on the presynaptic membrane and get internalized when the SVs are retrieved. Once endocytosed, the acidic vesicular lumen protonates cypHer molecules, which emit red fluorescence when excited at $640 \mathrm{~nm}$. Antibody incubation for 3-4 hrs leads to accumulation of a considerable amount of cypHer coupled antibodies in synaptic boutons, which exhibit a punctate staining (Fig. 3.16b). The fluorescence of cypHer tagged molecules on the presynaptic membrane is significantly silenced, only $\sim 30 \%$ is visible in neutral $(\mathrm{pH} 7.4)$ extracellular environment, thus allowing localization of functional boutons possible with considerable signal-to-noise ratio. Moreover, SV recycling occurs only in spontaneously active boutons, which makes this cypHer-based labeling specific for active boutons. 
(a)

(b)
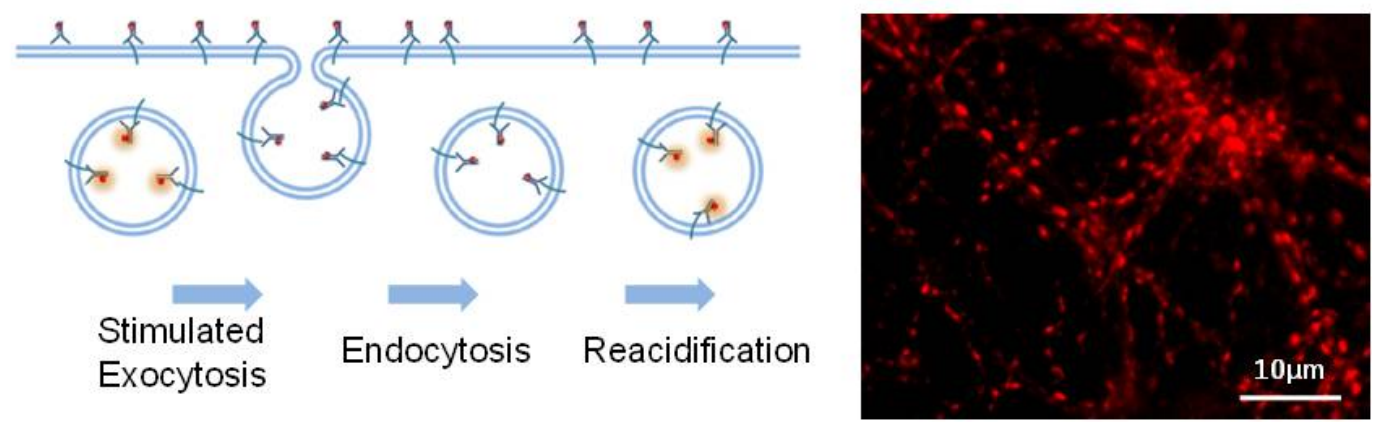

(c)

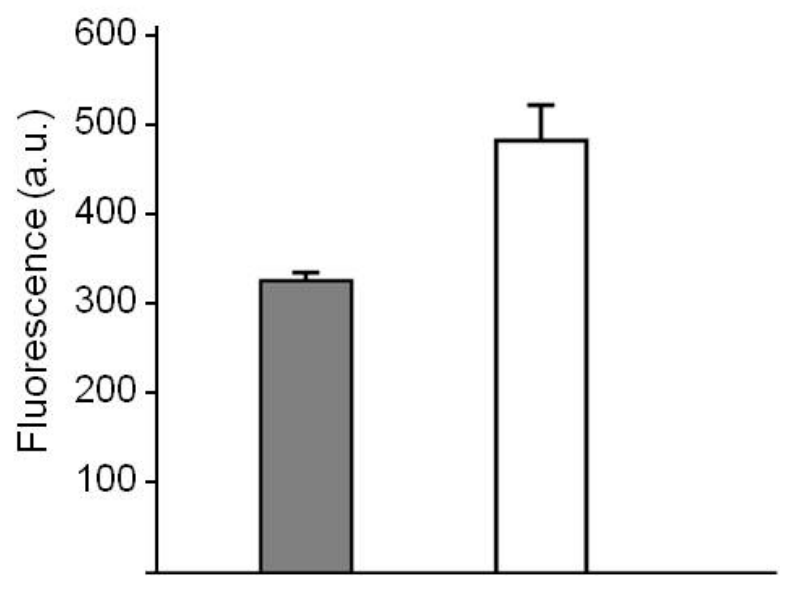

Figure 3.16: Live-cell labeling of hippocampal neurons with cypHer-coupled antibodies.

(a) Schematic illustration depicting fluorescence change in cypHer dye upon exo-endocytosis. CypHercoupled antibodies (red dots) bind to the extracellular/luminal domain of vesicular proteins (Syt1 or VGAT) and remain quenched due to the neutral extracellular $\mathrm{pH}$. The intravesicular acidic $\mathrm{pH}$ dequenches the cypHer dye and the fluorescence increases. Upon SV fusion and neutralization of cypHer dye the fluorescence declines. During endocytosis and reacidification the fluorescence signal recovers to the baseline.

(b) Fluorescence image of hippocampal neurons labeled with aVGAT-cypHer exhibit a distinct punctate pattern with each puncta typically representing individual active boutons.

(c) aSyt1-cypHer stained boutons are quenched using $\mathrm{NH}_{4} \mathrm{Cl}$ to estimate the total fraction of labeled Syt1 in the vesicular pool. The amplitude of the quenchable fraction after stimulation-induced (900 APs at 20 $\mathrm{Hz}$ ) uptake of aSyt1-cypHer (gray bar) is comparable to that after spontaneous labeling (white bar) at $37^{\circ} \mathrm{C}$ for 3-4 hrs. 
Antibody labeling reached saturation after incubation for 3-4 hrs. The labeling strategy used in this study does not block APs, with TTX, to exclusively measure spontaneous recycling. However, to test the efficiency of this labeling strategy the fraction of internalized aSyt1cypHer was compared to the fraction labeled with a stimulation-induced labeling protocol. Neurons were stained with aSyt1-cypHer in the presence of a strong stimulus such as 900 APs at $20 \mathrm{~Hz}$, which depletes the entire recycling pool of SVs in hippocampal boutons (Sankaranarayanan and Ryan, 2000). The neurons were then incubated at $37^{\circ} \mathrm{C}$ for $5-10$ min followed by extensive rinses to remove unbound antibodies. The internalized fraction of cypHer molecules was estimated by quenching the fluorescence with $\mathrm{NH}_{4} \mathrm{Cl}$, which equilibrates the $\mathrm{pH}$ across all membranes to 7.4 and therefore unmasks any cypHer-tagged molecules inside acidic compartments (Sankaranarayanan and Ryan, 2000). This quenchable fraction, which represents the population of aSyt1-cypHer internalized into SVs during the labeling time period, was comparable for both staining protocols (Fig. 3.16c). The better staining for spontaneous labeling can be explained by the more efficient epitope accessibility during the period of 3-4 hrs compared to the 5-10 min period used for the stimulation-induced labeling protocol (Fig. 3.16c). Moreover, aSyt1-cypHer labeling of neurons by spontaneous recycling (in the presence of TTX) targets the same pool of SVs as evoked (900 APs at $20 \mathrm{~Hz}$ ) labeling (Hua et al., 2010) contrary to recent studies (Chung et al., 2010; Fredj and Burrone, 2009).

\subsubsection{Stimulation-dependent exo-endocytosis measured using cypHer-coupled antibodies}

Once the boutons were labeled, the efficiency of the cypHer probe to monitor stimulationdependent exo-endocytosis was tested. Neurons were electrically stimulated by trains of APs delivered at a frequency of $20 \mathrm{~Hz}$. The cypHer fluorescence exhibited a sharp decline upon AP firing at individual boutons due to quenching of the vesicle resident cypHer coupled antibodies upon fusion and consequent neutralization at pH 7.4 (Fig. 3.17a). The cypHer fluorescence reached a minimum at the end of the stimulus followed by an exponential recovery due to dequenching of the internalized fluorophores by endocytosis and subsequent reacidification of SVs (Fig. 3.17b \& c). As is evident the cypHer approach provides a reverse fluorescence 
profile in comparison to the typical $\mathrm{pH}$ response, as expected from its opposite $\mathrm{pH}$-dependent fluorescence properties. Average traces of aSyt1-cypHer stained neurons exhibited typical stimulation-dependent fluorescence decay followed by an exponential rise (Fig. 3.17b). Selective labeling of inhibitory boutons was achieved by incubation with aVGAT-cypHer, which exhibited a similar punctate distribution after internalization of the probe. Upon stimulation, the aVGAT-cypHer stained inhibitory boutons showed similar fluorescence responses as aSyt1cypHer stained neurons (Fig. 3.17c).

(a)

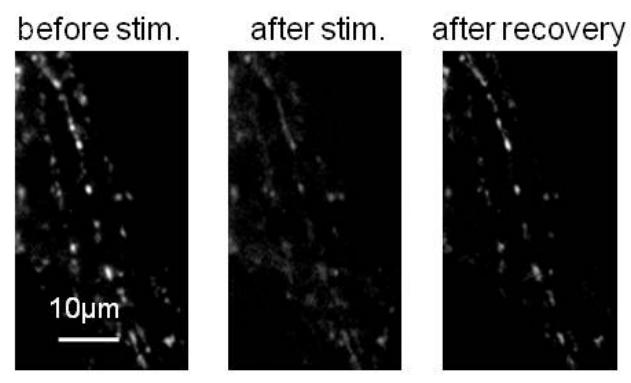

(b)

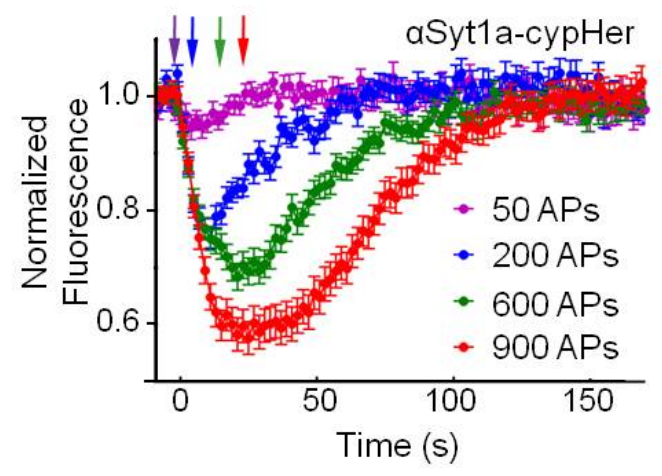

(c)

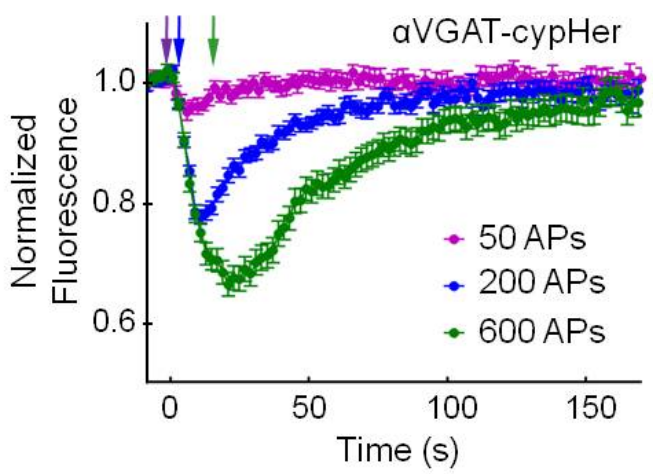

Figure 3.17: Fluorescence response of the cypHer probe upon exo-endocytosis.

(a) Fluorescence image of neurons stained with aVGAT-cypHer before and after stimulation (Stim.) with 900 APs.

(b) Average normalized fluorescence signal of aSyt1-cypHer stained boutons in response to different AP trains at $20 \mathrm{~Hz}$ ( $\mathrm{n}>50$ boutons for each stimulus). Error bars represent s.d.

(c) Average normalized fluorescence signal of aVGAT-cypHer stained boutons in response to different AP trains at $20 \mathrm{~Hz}$ ( $n>50$ boutons for each stimulus). Error bars represent s.d. 


\subsubsection{Size of the surface pool of synaptic vesicle constituents}

The fluorescence change of the cypHer signal scaled with the stimulus strength for both aSyt1-cypHer and aVGAT-cypHer labeled boutons (Fig. 3.17b \& c). The average peak fluorescence $(\Delta \mathrm{F})$ of aSyt1-cypHer stained boutons in response to 50,100 and 200 APs was calculated. This peak fluorescence reports the net difference between the kinetics of exo- and endocytosis during the stimulus. Previous studies based on $\mathrm{SpH}$ have shown that the initial rate of endocytosis post stimulus is independent of the stimulus strength and is similar to the endocytic rate during the stimulus period (Fernandez-Alfonso and Ryan, 2004; Sankaranarayanan and Ryan, 2000). Thus, we could estimate the pure exocytosis amplitude $\Delta \mathrm{F}$ by back-extrapolation of a linear fit to the initial post stimulus rate of endocytosis for each stimulus train (Fig. 3.18a \& b). Corrected $\Delta \mathrm{F}$ values, reflecting numbers of released vesicles, rise linearly with stimulus strength (Fig. 3.18b) as shown previously for $\mathrm{SpH}$ (FernandezAlfonso and Ryan, 2004; Sankaranarayanan and Ryan, 2000).

Up to $\sim 30 \%$ of $\mathrm{SpH}$ is stranded on the presynaptic membrane upon overexpression in hippocampal synapses (Dittman and Kaplan, 2006; Sankaranarayanan and Ryan, 2000; Wienisch and Klingauf, 2006). Endogenous SV proteins have also been shown to be present on the membrane comprising a 'surface reservoir' of SV constituents (Taubenblatt et al., 1999; Willig et al., 2006). To re-address this issue neurons were labeled with aSyt1-cypHer as described above. Since the surface resident cypHer molecules are quenched by the neutral extracellular environment, neurons were superfused with acidic buffer solution $(\mathrm{pH} 5.5)$ to dequench this surface pool. Using this method, the cypHer-coupled Syt1 molecules on the presynaptic membrane can be selectively unmasked without affecting the intracellular fraction. Fig. 3.18c shows the average cypHer fluorescence response to the acid pulse indicating the presence of aSyt1-cypHer on the bouton membrane. The amplitude of the fluorescence rise from individual boutons was plotted in a histogram (Fig. 3.18d). The distribution was well fit by a Gaussian curve (adjusted $R^{2}=0.98$ ) with a size of $49.46 \pm 1.56$ a.u. The total size of this surface pool was equivalent to the fluorescence change induced by $\sim 70$ APs (Fig. 3.18d 
inset). Therefore, the surface pool can support compensatory endocytosis of SVs for stimuli of up to 70 APs i.e. it is quite similar in size to the RRP.

(a)

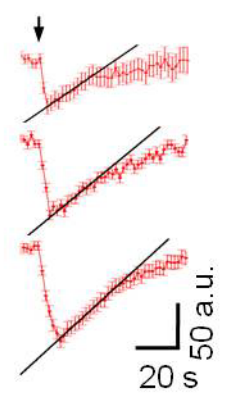

(c)

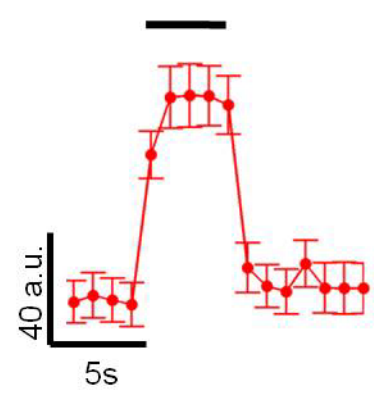

(b)

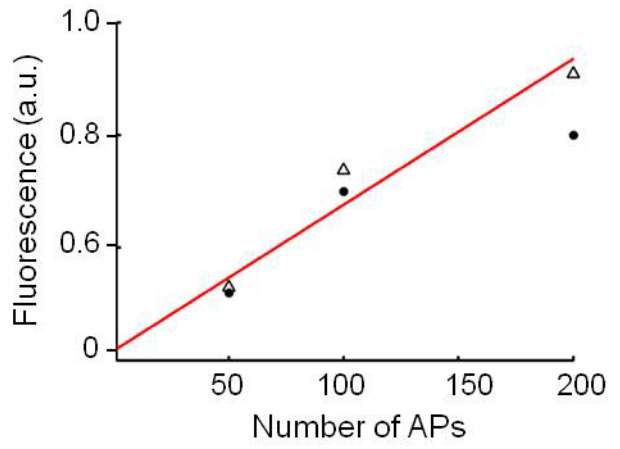

(d)

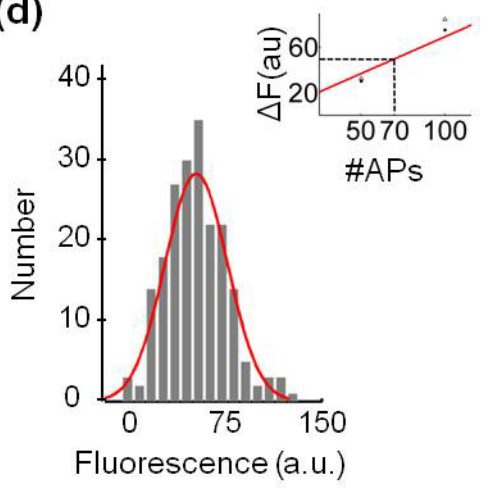

Figure 3.18: Dose-response curve to analyse the size of the surface pool.

(a) Averaged fluorescence traces of aSyt1-cypHer stained boutons in response to 50, 100 and 200 APs at $20 \mathrm{~Hz}$ ( $n>50$ boutons for each stimulus). Linear fit to the initial fluorescence recovery to estimate the endocytic rate constant during stimulus. Error bars represent s.d.

(b) Average peak change in fluorescence $(\Delta \mathrm{F})$ plotted as a function of AP number (filled circles) together with $\Delta \mathrm{F}$ corrected for endocytosis during stimulus (triangles) to estimate the net change in fluorescence exclusively due to exocytosis. These values were fit by a straight line (red).

(c) Fluorescence change in response to the acid pulse after labeling with aSyt1-cypHer. The increase in fluorescence indicates the size of the surface pool of Syt1 molecules.

(d) Distribution of fluorescence change upon application of an acid pulse. The solid red line represents a Gaussian fit (adjusted $R^{2}=0.99$ ) to the intensity distribution of fluorescence change in response to the acid pulse. Estimated mean size is $49.46 \pm 1.56$. Inset shows the size of the surface pool as estimated by the acid pulse is equivalent to the absolute fluorescence change triggered by 70 APs. 


\subsubsection{Dual-color measurements of vesicle recycling kinetics using SynaptopHluorin and cypHer-coupled antibodies}

CypHer-coupled antibodies provide a novel tool to label endogenous SV proteins and hence monitor exo-endocytosis at hippocampal boutons in real time. To compare these fluorescence profiles with that of the genetically encoded $\mathrm{pH}$ sensor, $\mathrm{SpH}$, a combination of $\mathrm{SpH}$ overexpression together with labeling of endogenous vesicle proteins, Syt1 and VGAT, with the cypHer-coupled antibodies was performed in hippocampal boutons. We performed dualcolor live-cell recordings with $\mathrm{SpH}$ (green) in one channel and cypHer-tagged antibodies (red) in the other with no bleed-through in between the channels, due to the wide spectral separation of the two fluorophores. Neurons transfected with $\mathrm{SpH}$ were labeled with cypHerconjugated antibodies aSyt1-cypHer or aVGAT-cypHer, by incubation at $37^{\circ} \mathrm{C}$ for 3-4 hrs (Material and Methods). Functional boutons labeled with aSyt1-cypHer colocalized with SpH overexpression as shown in Fig. 3.19a. Due to the low $\mathrm{SpH}$ transfection efficiency of $\sim 10 \%$ not all aSyt1-cypHer stained boutons co-express SpH. Trains of 200 APs were then delivered at $20 \mathrm{~Hz}$ and the fluorescence responses were examined. $\mathrm{SpH}$ signals showed typical fluorescence transients with the rising phase reflecting dequenching of $\mathrm{SpH}$ upon fusion/neutralization and the decay reflecting quenching of $\mathrm{SpH}$ by compensatory endocytosis and reacidification (Fig. 3.19b). In the red channel the aSyt1-cypHer responses of the same boutons showed mirrored signals as compared to $\mathrm{SpH}$, with a rapid decay phase (indicating exocytosis) followed by a slow exponential recovery (indicating endocytosis and reacidification) of the fluorescence signal. On fitting a monoexponential function to the recovery phase for both $\mathrm{SpH}$ and cypHer signals, similar time constants were estimated ( 23 $\mathrm{s}$ versus $\sim 22 \mathrm{~s}$; data not shown). This indicated that both probes label the same recycling pool of SVs and hence report the same kinetics of endocytosis and reacidification. Next, inhibitory $\mathrm{SpH}$ transfected boutons were labeled using the marker aVGAT-cypHer (Fig. 3.19c). About $32 \%$ of all SpH-expressing boutons were labeled with aVGAT-cypHer representing the fraction of inhibitory synapses in our cultures. Train stimulation with $200 \mathrm{APs}$ at $20 \mathrm{~Hz}$ induced similar fluorescence transients in both SpH and VGAT-cypHer channels (Fig. 3.19d). The time constant of fluorescence recovery was similar for both probes $(\sim 22 \mathrm{~s})$ consistent with the 
aSyt1-cypHer responses. Therefore, the kinetics of endocytosis and reacidification in inhibitory synapses is indistinguishable from the population response as probed with $\mathrm{SpH}$ and aSyt1cypHer.

(a)
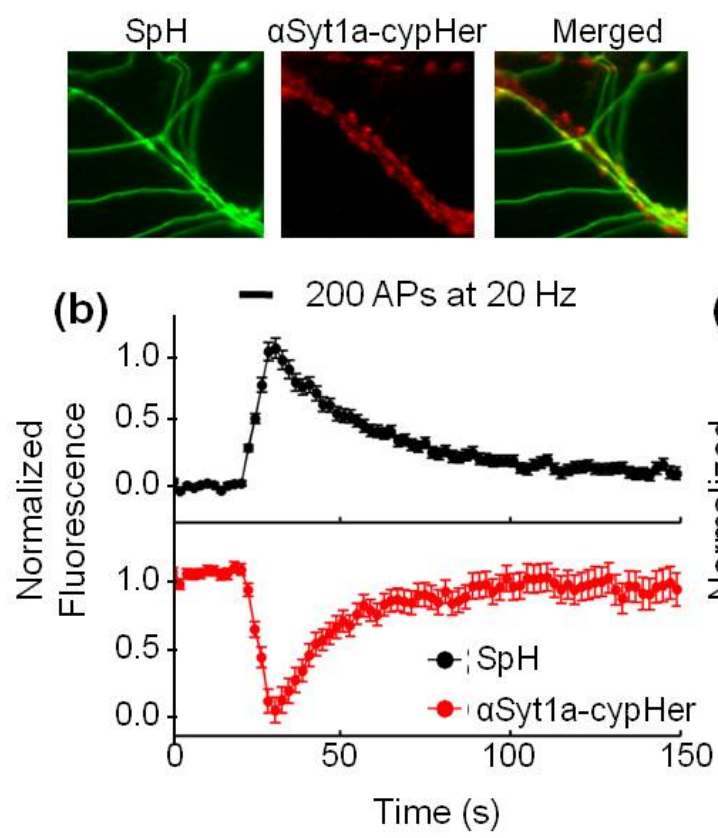

(c)

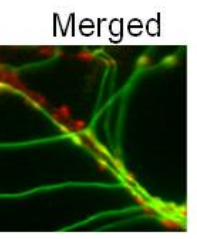

aVGAT-cypHer
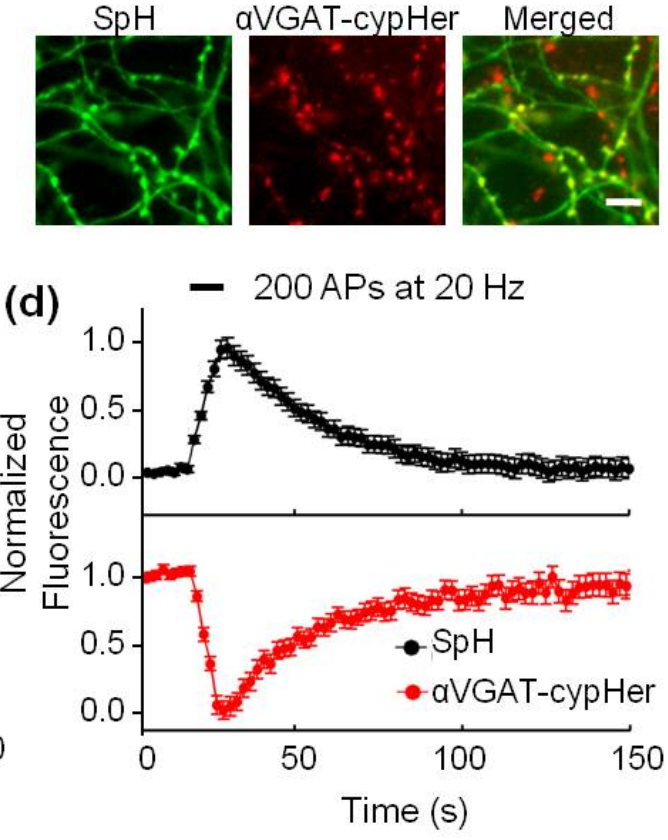

Figure 3.19: Comparison of SV recycling kinetics probed with $\mathrm{SpH}$ and cypHer-based technique.

(a) Staining of $\mathrm{SpH}$ transfected hippocampal neurons with aSyt1-cypHer. Fluorescence images show colocalization of individual boutons expressing $\mathrm{SpH}$ (green) and endogenous Syt1 (red) as labeled with aSyt1-cypHer.

(b) Average fluorescence signal of boutons during a stimulation with $200 \mathrm{APs}$ at $20 \mathrm{~Hz}$ for both $\mathrm{SpH}$ (green) and corresponding cypHer (red) channel ( $\mathrm{n}>50$ boutons). The fluorescence recovery of both $\mathrm{SpH}$ and cypHer traces were fit to a single exponential function. The time constant estimated by the fits were $\sim 23 \mathrm{~s}$ for $\mathrm{SpH}$ and $\sim 22 \mathrm{~s}$ for cypHer thus reporting very similar endocytic rates. Error bars represent s.d.

(c) Staining of SpH transfected hippocampal neurons with aVGAT-cypHer. Fluorescence images show colocalization of individual boutons expressing $\mathrm{SpH}$ (green) and the inhibitory synaptic marker VGAT (red).

(d) Average fluorescence responses to $200 \mathrm{APs}$ at $20 \mathrm{~Hz}$ measured by SpH and cypHer in parallel $(\mathrm{n}>$ 50 boutons). Monoexponential fit to the cypHer response yielded a time constant of $\sim 22 \mathrm{~s}$ similar to that estimated by SpH. Error bars represent s.d. Scale bar, $5 \mu \mathrm{m}$. 


\subsubsection{Kinetics of the readily retrievable surface pool of synaptic vesicle constituents}

Previous studies have shown that there is a surface reservoir of SV constituents which actively participates in stimulation-dependent exo-endocytic cycling and leads to molecular nonidentity between SVs exo- and endocytosed in response to the same stimulus (FernandezAlfonso et al., 2006; Wienisch and Klingauf, 2006). However, the kinetics of this functional surface pool has not been monitored in real time using a direct approach that selectively labels the endogenous native proteins. By engineering a TEV-cleavage site between the vesicle protein Syb2 and the $\mathrm{pHI}$, the fluorescence from the surface pool of $\mathrm{SpH}$ could be eliminated by TEV digest (Wienisch and Klingauf, 2006). Therefore to identify this pool, an indirect strategy was adopted in which SpH-TEV transfected neurons were co-labeled with aSyt1cypHer to label the endogenous Syt1 protein (Material and Methods). Next the neurons were incubated with TEV protease for 15 min as described previously (Wienisch and Klingauf, 2006), to silence the non-vesicular surface fluorescence of SpH-TEV transfected hippocampal boutons while keeping the aSyt1-cypHer molecules unperturbed. The RRP was depleted by delivering a train of $50 \mathrm{APs}$ at $20 \mathrm{~Hz}$ (Murthy and Stevens, 1999; Schikorski and Stevens, 1997). The SpH fluorescence transient displayed an instantaneous increase followed by little or no recovery (Fig. 3.20a, top panel) indicating that freshly exocytosed SV constituents including dequenched $\mathrm{SpH}$ is not retrieved by compensatory endocytosis (Wienisch and Klingauf, 2006). This indicates that the surface stranded pool of SV constituents are preferentially retrieved by the endocytic machinery rather than the freshly exocytosed material. Is this preferential uptake of membrane $\mathrm{SpH}$ a specific retrieval mechanism or a consequence of overexpression, which leads to missorted $\mathrm{SpH}$ and hence an exaggerated surface pool? To resolve this issue, the kinetics of the endogenous Syt1 labeled by the aSyt1-cypHer, was examined in the simultaneously recorded cypHer channel. The cypHer fluorescence transient showed a typical response with unperturbed fluorescence recovery indicating normal endocytosis and reacidification of SVs (Fig. 3.20a, bottom panel). Thus, the SV components $(\mathrm{SpH}$ and Syt1) being endocytosed upon exocytosis, are non-identical and derived from a functional surface pool of SV proteins present on the bouton membrane. Moreover, upon stronger or sequential stimulation the $\mathrm{SpH}$ fluorescence decay has been shown to gradually 
recover indicating that the surface pool of preexisting $\mathrm{SpH}$ has a limited capacity, necessitating additional endocytosis of freshly exocytosed SV constituents (Tabares et al., 2007; Wienisch and Klingauf, 2006).

Athough the previous experiment provides strong evidence in support of a surface pool of endogenous SV proteins, it was not possible to selectively label and measure the kinetics of this pool by itself. Therefore, to address this issue the vesicular cypHer fluorescence of $\alpha$ Syt1cypHer labeled SpH-transfected boutons was silenced by photobleaching at $640 \mathrm{~nm}$ for $5 \mathrm{~min}$. To minimize the effect of photobleaching aSyt1-cypHer on the surface pool of quenched Syt1cypHer molecules (since $30 \%$ remain unquenched at $\mathrm{pH} 7.4$ ) the neurons were superfused in an extracellular buffer of $\mathrm{pH} 8.5$ during the bleaching time (to maximize quenching), which was then replaced with neutral buffer $(\mathrm{pH} \mathrm{7.4)}$ just before stimulation. This way most of the vesicular Syt1-cypHer signal was silenced and a large degree of the surface fluorescence was preserved. The SpH channel remained unperturbed both in terms of its vesicular and surface signal. Stimulation with $50 \mathrm{APs}$ at $20 \mathrm{~Hz}$ induced typical fluorescence transients in SpHoverexpressing boutons with a rapid rise followed by an exponential decay (Fig. 3.20b, top panel). The $\mathrm{SpH}$ signal was used to map active boutons and their cypHer fluorescence response was then analyzed. The cypHer signal displayed little or no exocytic decrease upon stimulation indicating that most of the vesicular cypHer was prebleached (Fig. 3.20b, bottom panel). Notably, soon after the onset of stimulation the cypHer fluorescence markedly increased and reached a plateau, like a mirror image to the corresponding recovery phase of the $\mathrm{SpH}$ signal. This clearly suggested the preferential endocytosis of non-bleached Syt1cypHer molecules from the presynaptic membrane and their subsequent reacidification in the vesicular lumen (Fig. 3.20b, bottom panel). These data confirm the presence of SV constituents localized on the presynaptic membrane, which are readily retrieved upon exocytosis and hence constitute a distinct RRetP. Note, a small fraction of non-bleached vesicular cypHer molecules, due to incomplete bleaching, caused a slight delay or even small downward dip in the cypHer traces resulting in some remaining contribution of exocytosis. 
(a)

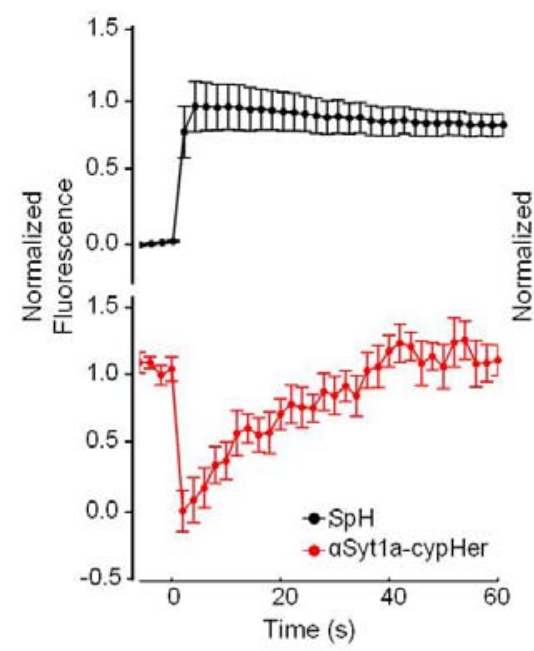

(b)

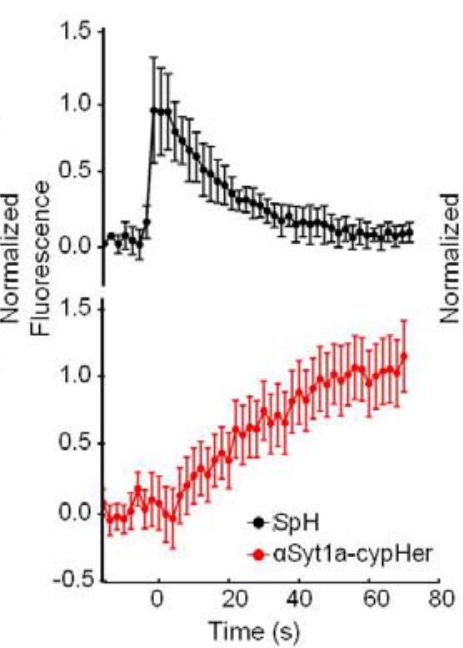

(c)

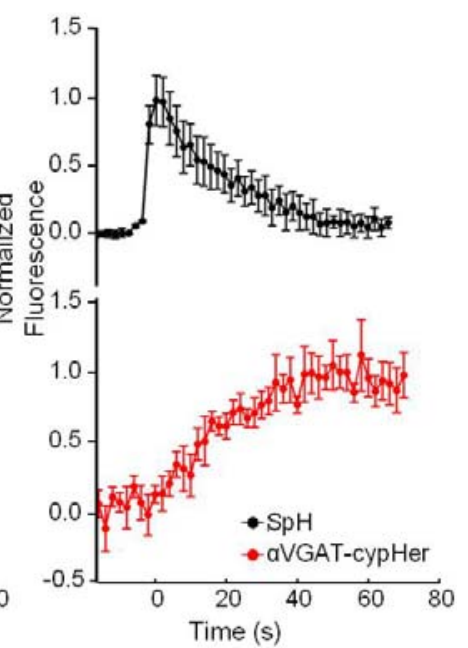

Figure 3.20: A readily retrievable surface pool of SV constituents.

(a) Cleaving plasma membrane-stranded $\mathrm{SpH}$ with TEV protease does not affect the cypHer signal at aSyt1-cypHer co-stained boutons. Average fluorescence profile in response to $50 \mathrm{APs}$ at $20 \mathrm{~Hz}(\mathrm{n}>100$ boutons). The $\mathrm{SpH}$ fluorescence transient (black) shows little or no recovery, while the cypHer signal (red) exhibits normal fluorescence recovery demonstrating endocytosis of pre-existing SV proteins (Syt1) rather than the freshly exocytosed SV proteins.

(b \& c) Photobleaching the cypHer signal at a pH of 8.5 for 5 min preferentially silences the vesicular pool of cypHer-tagged molecules. $50 \mathrm{APs}$ at $20 \mathrm{~Hz}$ induced typical SpH fluorescence transients (black) represented in the average trace ( $\mathrm{n}>100$ boutons). However soon after the stimulus the cypHer signal (red) for both (b) Syt1 and (c) VGAT, increases coincidentally with the SpH decay indicating endoyctosis of vesicle proteins from a RRetP resident on the bouton membrane. Error bars represent s.d.

To assess whether such a functional pool on the presynaptic membrane exists also for SV proteins with low copy number such as VGAT (Takamori et al., 2006), the above experiments were repeated using $\mathrm{SpH}$-transfected neurons co-stained with aVGAT-cypHer (Material and Methods). While upon stimulation with $50 \mathrm{APs}$ at $20 \mathrm{~Hz}$, the $\mathrm{SpH}$ transient showed normal compensatory endocytosis, the VGAT-cypHer signal increased and reached a plateau (Fig. 3.20c) indicating that even for low copy number SV proteins there exists a corresponding functional surface complement, which is preferentially recruited and internalized by the endocytic machinery. Therefore the presynaptic membrane acts as a major sorting platform, 
where SV constituents are recaptured and concentrated in the RRetP, which ensures the correct molecular composition of a SV.

\subsubsection{Size of the readily retrievable pool compared to the readily releasable pool}

During prebleaching of the vesicular cypHer molecules, a significant fraction of the surfaceresident cypHer molecules, although quenched by the alkaline $\mathrm{pH}$, also gets bleached since the fluorescence at alkaline $\mathrm{pH}$ is only reduced six- to seven-fold (compared to a 25 -fold fluorescence change for $\mathrm{SpH}$ ). The absolute size of this RRetP pool was quantified from the cypHer fluorescence increase observed in Fig. 3.20 upon 50 APs stimulation (Fig. 3.21). The sizes obtained were $19.5 \pm 6.2$ a.u. for Syt 1 and $17.7 \pm 2.1$ a.u. for VGAT. The RRP size estimated from the cypHer traces in Fig. 3.20a was $40.2 \pm 6.9$ a.u. for Syt1 and $34.8 \pm 3.6$ a.u. for VGAT (Fig. 3.21). These smaller size estimates of the RRetP could be solely attributed to partial bleaching of the surface pool, due to the small dynamic range of the cypHer dye (less than tenfold fluorescence drop from $\mathrm{pH} 5.5$ to 8.5 ), leading to a large underestimation of the RRetP size.

To circumvent the bleaching problem it was attempted to measure endocytosis from the RRetP by preferentially staining the surface-resident Syt1 molecules (Material and Methods). The results are shown in Fig.3.22 (amplitude quantification added to Fig. 3.21). This protocol of preferential surface labeling was able to reliably reproduce both the kinetics and amplitude of the RRetP retrieval like in Fig. 3.20. The absolute fluorescence increase upon the $1^{\text {st }}$ stimulus was now $53.7 \pm 4.8$ a.u. (Fig. 3.21), which was quite similar to the above estimates for the RRP (Fig. 3.20a) and within the range of surface pool (Fig. 3.18) sizes, equivalent to 70 APs. 


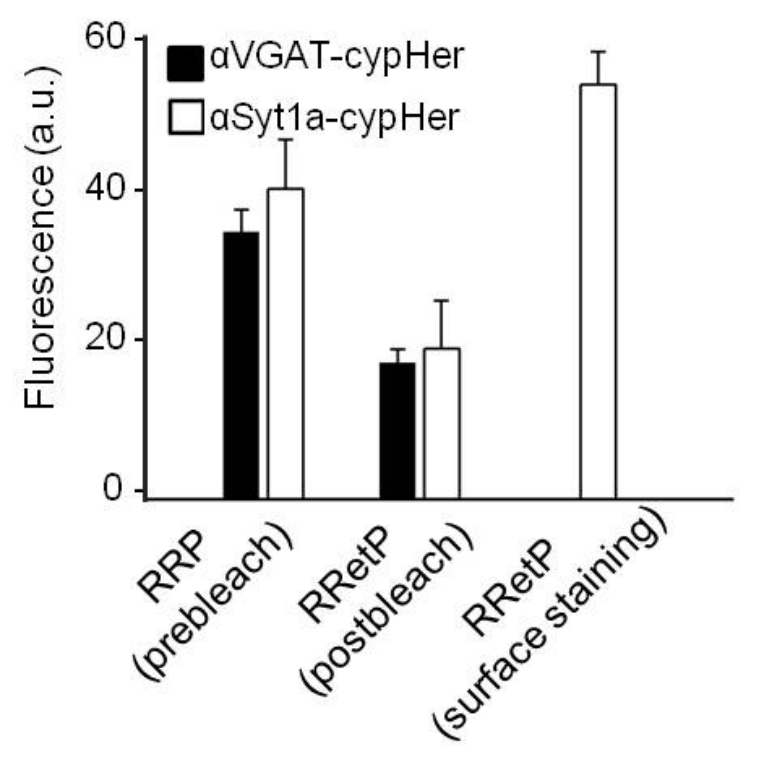

Figure 3.21: Size of the surface pool of SV constituents.

Comparison of RRP and RRetP sizes measured by different protocols for Syt1 and VGAT ( $\mathrm{n}>100$ boutons). Absolute fluorescence decreases in the cypHer transient upon $50 \mathrm{APs}$ at $20 \mathrm{~Hz}$ (before vesicular cypHer bleaching) are taken as measure for the size of the RRP (prebleach; $40.2 \pm 6.9$ a.u. for Syt1 and $34.8 \pm 3.6$ a.u. for VGAT). Absolute fluorescence increases on $50 \mathrm{APs}$ at $20 \mathrm{~Hz}$ (Fig. 3.20b \& c; cypHer traces) post bleach can be taken as lower bounds for the RRetP sizes (postbleach; $19.5 \pm 6.2$ a.u. for Syt1 and $17.7 \pm 2.1$ a.u. for VGAT). A better estimate for the RRetP size was obtained by preferential labeling of the Syt1 surface pool and subsequent quantification of the fluorescence increases (Fig. 3.21a), in response to 50 APs (surface staining; $53.7 \pm 4.8$ a.u.). Error bars represent s.e.m.

\subsubsection{Reuse of the readily retrievable pool upon sequential stimulation}

Can the RRetP be re-released upon repeated stimulation? To answer this question a second bout of stimulus was elicited $60 \mathrm{~s}$ later in the previous experiment to the surface Syt1-labeled boutons to monitor the re-release of the surface pool (Fig. 3.22). The cypHer fluorescence responses indeed displayed a fast drop due to partial exocytosis of the meanwhile recycled RRetP followed by an endocytic increase (Fig. 3.22a). Released fractions were $27.6 \pm 5.1 \%$ for 50 APs and $49.7 \pm 9.2 \%$ for 200 APs stimulation (Fig. 3.22b) in very good agreement with similar experiments performed using FM styryl dyes as tracers (Vanden Berghe and Klingauf, 2006). However, somewhat unexpected, the endocytic increases for the second stimulus 
clearly exceeded the exocytic drops (Fig. 3.22). This overshoot most likely reflects two effects. First, 50 APs might not fully deplete the RRetP (Fig. $\mathbf{3 . 1 8}$ suggest a capacity of rather 70 APs). Second, during staining some spontaneously recycling SVs were labeled that were exocytosed during the first stimulus (slowing somewhat the endocytic kinetics), therefore repopulating the RRetP (Fig. 3.22). Thus, the RRP appears to be counterbalanced by a RRetP of similar size.

(a)

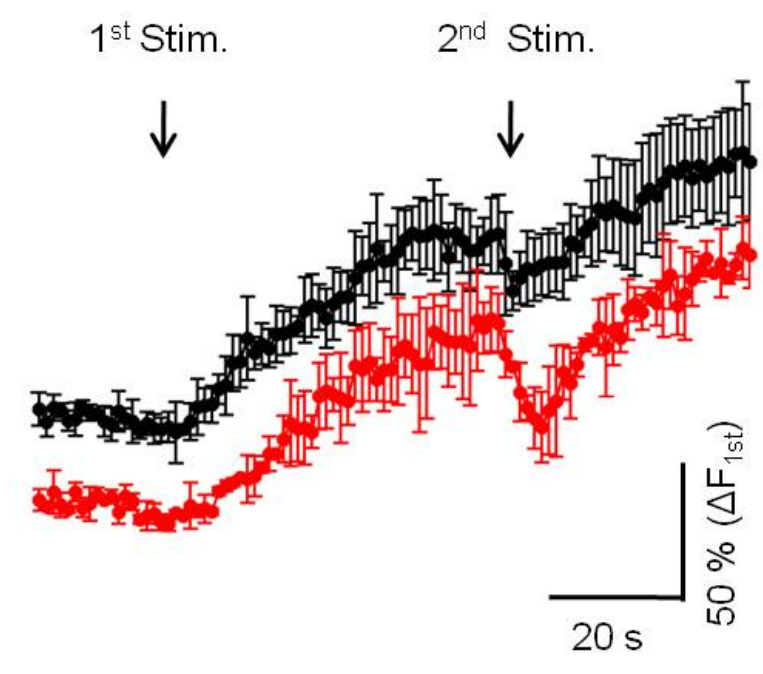

(b)

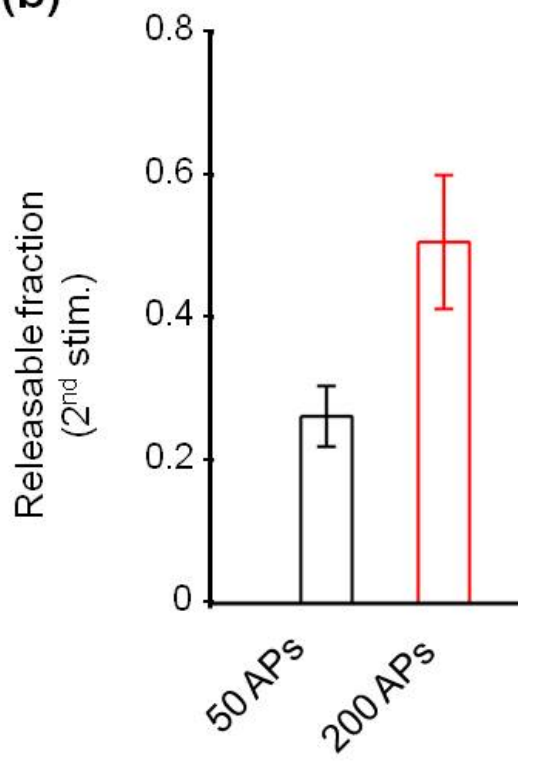

Figure 3.22: Sequential stimulation reveals reuse of the readily retrievable pool.

(a) Preferential staining of the surface pool of Syt1. Average fluorescence profile of boutons ( $n>100)$ stimulated first with $50 \mathrm{APs}$ at $20 \mathrm{~Hz}$ followed by a second stimulation (Stim.) with either 50 (black) or 200 APs at $20 \mathrm{~Hz}$ (red). In response to the second stimulus a marked fraction of the RRetP recycled in response to the first stimulus is released. Error bars represent s.d.

(b) Released fractions of the recycled RRetP (after first stimulus) in response to a second stimulus of 50 (27.6 $\pm 5.1 \%$; black) and 200 (49.7 $\pm 9.2 \%$; red) APs. Error bars represent s.e.m. 


\section{DISCUSSION}

\subsection{Two SNARE complexes are sufficient for vesicle fusion during fast synaptic transmission}

Recently single molecule techniques have been applied to cell biology of the synapse to count and localize GFP fused biomolecules and also determine their density in vivo (Balaji and Ryan, 2007; Chiu et al., 2002; Chiu et al., 2001; Sugiyama et al., 2005; Ulbrich and Isacoff, 2007). In the first part of this thesis I have applied such high-resolution fluorescence measurements towards understanding the precise stoichiometry of SNARE complexes sufficient to execute SNARE-dependent membrane fusion. By overexpressing a pHdependent switchable reporter, $\mathrm{pHI}$, fused to the SV SNARE Syb2 $(\mathrm{SpH})$, in hippocampal neurons single SV fusion was monitored (Fig. 3.3). The fluorescence contribution of a single fusing SV was calibrated with single $\mathrm{pHI}$ molecule measurements performed on isolated $\mathrm{pHI}$ molecules immobilized in a polyacrylamide gel (Fig. 3.5). This enabled the determination of the exact number of $\mathrm{SpH}$ proteins inserted per SV. The $\Delta \mathrm{F}$ distributions from optical recordings of single SV fusion appeared quantized where single $\mathrm{pHI}$ molecules could be resolved as distinct peaks of mean size equivalent to the single $\mathrm{pHI}$ molecule fluorescence (Fig. 3.4 and Fig. 3.5). To estimate the minimum copy number of $\mathrm{SpH}$ required for rescuing fusion of an individual SV, SpH was overexpressed on a genetic null background of Syb2/Ceb DKO hippocampal boutons. The $\Delta \mathrm{F}$ distributions, obtained from single vesicle $\mathrm{SpH}$ fluorescence measurements in DKO neurons, exhibited a dramatic absence of the one molecule peak (Fig. 3.9), which clearly defined the lower bound of two SNARE (SpH) molecules required to evoke fusion.

\subsubsection{Overexpression of pHluorin-fusion chimeras}

Previous studies using overexpression of the canonical $\mathrm{pHI}$-based reporter, such as $\mathrm{SpH}$, have reported an exaggerated fraction of these fusion proteins at the presynaptic membrane compared to native conditions. Such differences in the relative abundance and distribution of the $\mathrm{pH}$ chimeras have questioned the use of these probes for studying exo-endocytosis. Therefore, it was important to determine how many copies of these pHI-tagged SV proteins 
get incorporated into SVs upon transient overexpression. Systematic calibration of single SV fluorescence signals with single molecule bleaching analysis of purified and isolated $\mathrm{pHI}$ protein gave a direct measure of the actual number of reporters incorporated per SV (Fig. 3.4 and Fig. 3.5). These measurements indicate that on average very few (about two - three) copies of all the reporters (i.e. SpH, Syp-pHI or vGlut-pHI) get targeted per SV with a considerable fraction of SVs having only one copy (Fig. 3.4, Fig. 3.5 and Fig. S2). Thus, at the level of individual SVs there seems to be a major underexpression of these proteins. Moreover, this average copy number remains unaltered despite the type of promoter used to drive expression. This result was quite consistent with previous single vesicle optical studies where similar copy numbers of overexpressed $\mathrm{pHI}$-tagged SV proteins have been suggested (Balaji and Ryan, 2007; Fernandez-Alfonso et al., 2006; Gandhi and Stevens, 2003). Compared to previously reported endogenous copy numbers of SV proteins (Takamori et al., 2006), the number of corresponding pHI fusion protein targeted per SV in this study is strikingly low, which argues against any potential overexpression artifact associated with these probes for studying SV exo-endocytosis. Likewise, my experiments with the exogenous cypHer-based probe clearly demonstrate that $\mathrm{SV}$ recycling is identical for overexpressed and endogenous SV proteins.

\subsubsection{Regulation of synaptic vesicle protein stochiometry and release probability}

Given that there are 70 copies of Syb2 expressed per SV (Takamori et al., 2006), one would expect a larger quantal size for $\mathrm{SpH}$ in the recordings from WT neurons (Fig. 3.4). However, due to a fixed stoichiometry of SV proteins and competition with endogenous Syb2, there might not be empty slots for $\mathrm{SpH}$ to fill in the SV. Surprisingly, in the absence of the endogenous 70 Syb2 molecules (in Syb2/Ceb DKO neurons), the average copy number of $\mathrm{SpH}$ per SV still remains identical (two - three copies) indicating that incorporation of SpH into SVs is not slot-dependent (Fig. 3.4 and Fig. 3.9). Does this indicate a gross overestimation of copy numbers by the proteomic analysis of isolated SVs? A recent study based on single molecule fluorescence quantified the average number of SV proteins in isolated SVs and found a relatively low number $(\sim 10)$ of Syb2 molecules per SV and a high variance in Syb2 
copy numbers among SVs (Mutch et al., 2011). Therefore the stochiometry of SV proteins, in terms of Syb2 density, seems to be rather weakly regulated. Due to this high variance in the integration of SpH into SVs, there might be a proportion of 'dead-end' SVs in the DKO rescue boutons that have only one copy of $\mathrm{SpH}$ and are thus incapable of evoked fusion. Therefore, this population of non-fusing SVs might 'physically' hinder release at the AZ and lower the overall $\mathrm{P}_{\mathrm{r}}$. However, this would not be expected to have a dramatic effect in my measurements: First, since the single vesicle fluorescence distribution appears skewed, this 'dead-end' SV fraction is considerably small in comparison to SVs containing multiple SpH and fails to significantly affect the synaptic release properties (Fig. 3.9). Secondly, when both single AP fluorescence distributions are coarsely binned (quantal size reflect average copy numbers), they appear indifferent between WT and DKO rescue conditions with identical quantal size indicating that the average number of two - three SpH per SV remains unaltered (Fig. S2). Therefore it seems the two and three-molecule peak together in the $\Delta \mathrm{F}$ distributions for both SpH-expressing WT and DKO neurons (Fig. 3.4 and Fig. 3.9) are relatively dominant and contribute significantly towards the first quantal peak in the coarsely binned distribution (Fig. S2). Thus, SVs with two or three SpH fuse more frequently in WT or DKO boutons. Thirdly, when the fluorescence contribution of the single molecule peak is weighted against that of the other higher-order peaks by plotting an intensity-weighted distribution, it results in a minor difference of $\sim 10 \%$ between the WT and DKO boutons (Fig. 3.11). Thus, the effect of non-fusing SVs on release property might be partially compensated by homeostatic mechanisms at the presynapse which might upregulate the $\mathrm{P}_{\mathrm{r}}$. Such presynaptic homeostatic compensation is quite often observed within several minutes of postsynaptic receptor inhibition (Davis, 2006). Over a course of two weeks post-transfection, the synapse could potentially reorganize by increasing the number of release sites or altering the presynaptic $\mathrm{Ca}^{2+}$ current to account for low $\mathrm{P}_{\mathrm{r}}$ caused by presence of non-fusing $\mathrm{SV}$ s with only one $\mathrm{SpH}$ molecule. 


\subsection{3 pHluorin maturation efficiency; implication for fluorescence amplitude estimation}

The surprisingly low number of $\mathrm{SpH}$ required to rescue evoked fusion raises concerns over whether all the $\mathrm{pHI}$ moieties are functional or not. If indeed, there is a significant fraction of non-fluorescent $\mathrm{SpH}$, where the $\mathrm{pHI}$ is misfolded or not fully matured, it might lead to an underestimation of the actual number of $\mathrm{SpH}$ molecules per SV. However, the main results in this study point toward a rather low fraction of fluorescently silent SpH (Fig. 3.9). Since the occurrence of a non-fluorescent $\mathrm{SpH}$ molecule is random, it would be expected to equally affect the frequency of all quantal peaks and therefore would mask the selective reduction of the one-molecule peak in DKO rescue condition (Fig. 3.9). Hence, the dramatic reduction of the one-molecule SpH peak (Fig. 3.9) rather indicates a relatively high efficiency of GFP folding and maturation. Moreover, previous studies have reported that the fraction of misfolding for GFP is very low and consequently the chromophore formation efficiency of GFP fusion proteins is very high ( $80-90 \%$ ) (Patterson et al., 1997; Sugiyama et al., 2005; Ulbrich and Isacoff, 2007) - too high to result in any underestimation of my measurements. An elegant study has determined the efficiency of chromophore formation for postsynaptic GFP fusion proteins in hippocampal neurons (Sugiyama et al., 2005). Based on anti-GFP immunostaining and single molecule calibration it was suggested that $\sim 70-80 \%$ of the fluorescent proteins are functional, a lower limit considering that not all the epitopes would be accessible by the antibody (Sugiyama et al., 2005). To cause any appreciable underestimation of the SNARE molecules in my single vesicle measurements and hence account for the lack of the single molecule peak in Fig. 3.9 the folding efficiency of GFP has to be considerably lower.

$\mathrm{pHI}$ is attached to the ectodomain next to the TMD. Thus GFP on the C-terminus has ample time to fold during trafficking of the SV to the synapse. However, the folding of GFP should be fully independent of the SNARE motif structure on the other side of the membrane which might be still rather unstructured and might only completely fold when part of the SNARE complex. This independence is demonstrated by crystallographic studies, where only the cytosolic SNARE motifs, i.e. with TMDs chopped off, were used (Fasshauer et al., 1998b; Rizo and Rosenmund, 2008; Sutton et al., 1998). More importantly, one would expect the one-molecule 
peak to be unaltered in presence of misfolded non-fluorescent $\mathrm{SpH}$ molecules contrary to the situation represented in Fig. 3.9, since a fraction of the participating $\mathrm{SpH}$ molecules in the SNARE complex would always go undetected. Thus, the proportion of non-fluorescent SpH molecules can be well accounted for by the residual one-molecule peak of the intensity distribution obtained from SpH overexpression in DKO boutons (Fig. 3.9).

\subsubsection{Effects of local environment on pHluorin fluorescence}

The similarity of the $\mathrm{SpH}$ fluorescence in the neuronal measurements and in vitro indicates that the fluorophore environment does not have a considerable effect on its fluorescent property (Fig. 3.4 \& Fig. 3.5). This is quite consistent with the tightly constructed $\beta$ barrel structure of GFP which serves the role of protecting the chromophore well. Hence, the fluorescence properties of the chromophore, which is largely protected by the rigid shell of GFP, are independent of ionic strength, polarity of solution, and other conditions that might be encountered in living cells (Lippincott-Schwartz et al., 1999; Piston et al., 1999; Tsien, 1998). Careful single molecule calibrations have convincingly shown that GFP fluorescence intensity is unperturbed in the cellular environment even after fixation with 4 \% PFA (Brock et al., 1999; Chalfie et al., 1994; Chiu et al., 2002). Fluorescence characterstics of single GFP molecules have been extensively studied by immobilizing in aerated aqueous polymer gels where the 'intrinsic intensity' remains unaltered (Balaji and Ryan, 2007; Chiu et al., 2002; Chiu et al., 2001; Dickson et al., 1997; Hack et al., 2000; Kubitscheck et al., 2000). Thus the fluorescence property of GFP does not seem to be altered in my recording conditions.

\subsubsection{Participation of other v-SNARE molecules}

Fast $\mathrm{Ca}^{2+}$-triggered fusion in Syb2-deficient hippocampal neurons is diminished more than 100-fold (Schoch et al., 2001) and it has been convincingly shown to be the predominant isoform of v-SNAREs in these synapses. Besides Syb2, other closely related v-SNARE isoforms such as Ceb and Syb1 can mediate exocytosis, but they appear to be absent from the forebrain synapses (McMahon et al., 1993; Schoch et al., 2001; Trimble et al., 1990). However, in this study I use Syb2/Ceb DKO neurons thus eliminating both major v-SNARE isoforms. Another study on this DKO performed capacitance measurements on chromaffin 
cells (where both Ceb and Syb2 can catalyse evoked fusion) and showed that $\mathrm{Ca}^{2+}$-evoked secretion from these cells is nearly abolished. Treatment with Tetanus toxin (TeNT) led to no further reduction of the signal excluding Syb1 from a role in mediating fusion (Borisovska et al., 2005). Thus it is unlikely that other Syb isoforms can compensate for the lack of Syb2 by more than $1 \%$ for SV fusion. However, other non-cognate SNARE interactions might occur, which could explain the residual (less than $1 \%$ ) fusion observed in the DKO neurons (Fig. 3.9). In my data the dramatic reduction of the one-molecule peak (Fig. 3.9) is indicative of a sole dependence on Syb2 for evoked fusion.

\subsubsection{Docking and tethering of synaptic vesicles}

Previous studies have suggested that the initial docking and tethering of vesicles with the plasma membrane could be mediated by trans-SNARE pairing where the docked state could represent a partially zippered trans-SNARE complex (Reese et al., 2005; Verhage and Sorensen, 2008). The findings in this study provide new insight into the process of vesicle docking, priming and fusion during fast synaptic transmission. With a minimum of two $\mathrm{SpH}$ molecules per SV it is difficult to imagine that SVs could dock with their two SNAREs already pointing to the plasma membrane ready to engage and lock with t-SNAREs. Rather, other SV proteins could mediate the initial docking and tethering. The two SV SNAREs can freely diffuse within the SV membrane and should therefore be positioned randomly on the SV surface during the process of docking. However, once the SV is docked to the presynaptic membrane, the v-SNAREs diffuse to the base of SV and engage in SNARE pairing. Taken together, my findings imply that the initial docking is Syb2-independent and could rather be driven by other factors such as Munc18-1 (Voets et al., 2001), Syx1A (de Wit et al., 2006) or, as shown recently, Syt1 (de Wit et al., 2009). 


\subsubsection{Molecular nature of the fusion pore}

During regulated exocytosis, merger of the two membranes leads to the formation of an aqueous fusion pore whose physical properties have long been debated (Jackson and Chapman, 2008). As a result two contrasting schools of thought have emerged depending on whether the pore is lined by lipid or protein. Based on my results the fusion pore is most likely composed of lipids, two TMDs of Syb2, and two TMDs of Syx1A (Fig. 4.1). This implies that the inner fusion pore is predominantly lined by lipids instead of SNARE TMDs being arranged like barrel staves around the pore (Montecucco et al., 2005; Tokumaru et al., 2001; Weber et al., 1998). However, one cannot exclude the possibility that TMDs contributed by other integral membrane proteins such as Syt1, Syp or ion-channels may result in the formation of a proteinaceous fusion pore after its initial inception.
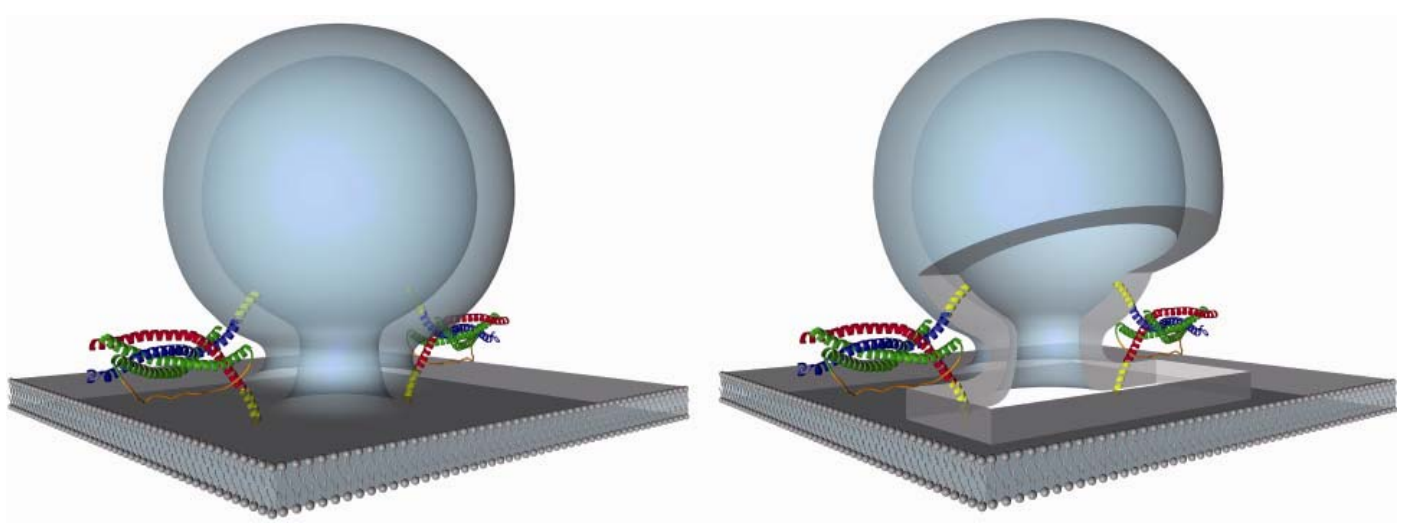

Figure 4.1: An illustration of SV fusion driven by two SNARE complexes during neuroexocytosis.

Assembly of two SNARE complexes by coil-coiling of the vesicle SNARE protein Syb2 (blue) and the two presynaptic membrane SNARE proteins Syx1A (red) and SNAP-25 (green) drives fusion. Each SNARE complex consists of four $\alpha$-helices aligned in parallel, with Syb2 and Syx1A contributing one helix and SNAP-25 contributing two helices. The transmembrane regions of the SNARE proteins are depicted in yellow (better seen in the version with a cut-open fusion pore on the right).

Courtesy: H. Sebesse and C.P. Adam. 


\subsubsection{Kinetics and energetics of vesicle fusion}

The finding that such a low copy number of Syb2 can rescue evoked fusion raises the possibility that the kinetics of synaptic transmission, observed in SpH-overexpressing DKO neurons, may be slower as compared to normal physiological neurosecretion where there might be up to 70 copies of Syb2 present per SV (Takamori et al., 2006). However, an earlier as well as a recent study have shown, that overexpression of $\mathrm{N}$ - or C-terminal GFP fusion constructs of Syb2 in Syb2 KO hippocampal neurons can fully rescue the amplitude and the fast kinetics of evoked excitatory postsynaptic potentials in Syb2 KO neurons (Deak et al., 2006; Guzman et al., 2010). Thus, the low copy numbers of SpH per SV appear to be sufficient to fully restore the kinetics of evoked fusion in DKO boutons.

SNARE assembly is believed to generate sufficient energy to drive membrane fusion (Hanson et al., 1997; Hu et al., 2003; Lin and Scheller, 1997). Recent studies using a surface force apparatus indicate that the stabilization energy of a single partially assembled neuronal SNARE complex is $\sim 35 k_{B} T$, which corresponds to the energy required for hemifusion of lipid bilayers (40-50 k $\left.k_{\mathrm{B}} \mathrm{T}\right)$ (Cohen and Melikyan, 2004; Kozlovsky and Kozlov, 2002; Li et al., 2007). Moreover, using isothermal titration calorimetry, the free energy estimated for the assembly of individual SNARE complexes was found to be sufficient for membrane fusion (Wiederhold and Fasshauer, 2009). Thus, assembly of one SNARE complex can in theory drive fusion. Indeed, a recent study based on in vitro FRET experiments indicate that liposomes bearing a single SNARE molecule are still capable of fusion with other liposomes or with purified SVs (van den Bogaart et al., 2010). Why in vivo more SNARE complexes are needed than in vitro remains to be elucidated. One reason might be the different time scales on which SV fusion and in vitro fusion proceed: while AP-triggered SV fusion in my experiments is completed within ms, in vitro fusion takes sec, indicating the presence of another very slow rate-limiting step upstream of SNARE complex formation in vitro. A trivial reason might also be that Syb2 is trafficked to SVs and to the synapse as dimers (Fdez et al., 2008). In this case, however, I would expect a suppression of not only the one-molecule but also the three-molecule peak in the amplitude histogram of SpH expressing DKO neurons (Fig. 3.9), which was not observed. In conclusion, 
the finding that two SNARE-complexes are sufficient and necessary for SV priming and fast $\mathrm{Ca}^{2+}$-triggered exocytosis fundamentally revises our understanding of SNARE-mediated fusion pore formation and membrane fusion. 


\subsection{Minimal number of Synaptotagmin1 required to trigger fusion}

The single vesicle optical measurements demonstrate a similar amplitude distribution of Syt1$\mathrm{pHI}$ expression in $\mathrm{KO}$ and $\mathrm{WT}$ boutons with several equidistant peaks of width equivalent to a single pHI molecule (Fig. 3.13 and Fig. 3.14). Unlike the $\mathrm{SpH}$ distribution in the Syb2/Ceb DKO neurons, the single molecule peak is unperturbed when Syt1-pHI is expressed in Syt1 KO neurons indicating that one copy of Syt1-pHI can rescue evoked release (Fig. 3.14). In principle, a single Syt1 molecule can bind five $\mathrm{Ca}^{2+}$ ions and therefore can fulfill the $\mathrm{Ca}^{2+} \mathrm{co}-$ operativity for single SV release. But to ensure the validity of my results, it needs to be tested whether Syt1-pHI is indeed able to rescue the kinetics/synchronicity of release with patchclamp physiology. Although other isoforms of Syt, such as Syt2 and Syt9, localized on SVs can rescue synchronous release when overexpressed on the null background, their endogenous levels are quite low and not sufficient to mediate evoked fusion in the absence of Syt1 (Pang et al., 2006; Xu et al., 2007). Furthermore, cleavage of Syt1-pHI would result in a major underestimation of Syt1 copy numbers by filling SVs with unlabeled Syt1. Therefore, proteolytic cleavage of Syt1-pHI upon overexpression needs to be checked using western blot analysis of the cell lysates with an anti-Syt1 antibody. Despite these caveats, a previous study based on charge neutralization of the $\mathrm{Ca}^{2+}$ binding $\mathrm{C} 2$ domains in Syt1 proposed that as little as two Syt1 molecules may be sufficient to initiate fusion (Stevens and Sullivan, 2003), which is close to my estimate of one Syt1 molecule per SV. If indeed one Syt1 molecule is sufficient to evoke release, then the quantitative model of $\mathrm{Ca}^{2+}$ co-operativity originally proposed (Dodge and Rahamimoff, 1967) could be attributed to the co-operative binding of $\mathrm{Ca}^{2+}$ to different sites within a single Syt1 molecule. 


\subsection{A readily retrievable pool of synaptic vesicle constituents}

In the second part of the thesis the functional surface pool of endogenous SV constituents, the so-called readily retrievable pool (RRetP) was identified in hippocampal synapses, and its kinetics were directly monitored using a novel exogenous $\mathrm{pH}$-sensitive marker, cypHer5E dye, coupled to antibodies against the luminal domains of Syt1 and VGAT (Fig. 3.16). Due to the opposite $\mathrm{pH}$-dependent fluorescence properties of the cypHer dye as compared to $\mathrm{pH}$ (cypHer is visible at a pH of 5.5 and quenched at neutral $\mathrm{pH}$ ) and the wide spectral separation (cypHer Ex: 640 nm; pHI Ex: 488 nm), it could be used as a parallel and independent read-out to reliably monitor exo-endocytosis during SV recycling in hippocampal synapses in real time

(Fig. 3.17 and Fig. 3.19). Endocytic time constants measured using cypHer-conjugated antibodies against Syt1 and VGAT or SpH yielded comparable results indicating that both probes do not interfere with the endocytic machinery (Fig. 3.19). Moreover, the retrieval machinery does not distinguish between native and overexpressed proteins. To visualize the spatial and temporal kinetics of the RRetP, endogenous SV proteins on the presynaptic membrane were either selectively labeled by preferential surface staining with cypHercoupled antibodies or the vesicular cypHer fluorescence was silenced by prebleaching while keeping the plasma membrane cypHer molecules relatively unperturbed (Fig. 3.20 and Fig. 3.22). Upon modest stimulation the preferentially labeled surface pools of Syt1 and VGAT molecules were readily endocytosed with little or no contribution from the freshly exocytosed SV constituents (Fig. 3.20 and Fig. 3.22). The kinetics of retrieval from the RRetP followed the usual time course of clathrin-mediated endocytosis and could fully compensate for the depletion of the RRP in these synapses (Fig. 3.18 and Fig. 3.21). Stronger stimulation exhausts this pool and leads to active recruitment of newly added SV components (Wienisch and Klingauf, 2006). Once endocytosed, this surface pool was recycled back into the RRP for further rounds of use (Fig. 3.22). Overexpression of SV proteins, such as $\mathrm{SpH}$, does not affect the size, capacity or kinetics of this RRetP indicating that this is not a mere consequence of surface-stranded proteins but rather a fundamental phenomenon crucial for SV recycling. 


\subsubsection{A novel exogenous tracer for studying exo-endocytosis of endogenous vesicle proteins}

Most optical assays used to study exo-endocytosis in synapses rely on non-specific membrane tracers such as FM dyes or on overexpression of genetic probes such as $\mathrm{pHI}-$ fusion chimeras. Due to the high background fluorescence and slow departitioning rates of FM dyes, it is difficult to measure fast kinetics and make conclusions about different modes of SV recycling (Smith et al., 2008). On the other hand possible overexpression artifacts associated with pHI-fusion proteins make it difficult to draw conclusions about the cellular distribution and dynamics of the native SV proteins. Thus, it is essential to study the trafficking of SV proteins in their native environment and more so in real time. Using cypHer5E-conjugated antibodies against Syt1 and VGAT we achieved specific labeling of these endogenous SV proteins (Fig. 3.16). Antibody uptake into SVs during the $3-4 \mathrm{hrs}$ incubation at $37^{\circ} \mathrm{C}$, however, might be mediated to a large degree by spontaneous recycling, and thus might label a different SV pool in comparison to stimulation-induced uptake (Fig. 3.16) (Fredj and Burrone, 2009; Groemer and Klingauf, 2007; Sara et al., 2005). But analysis of the spontaneous and evoked SV recycling, using aSyt1-cypHer, revealed that both modes of release draw upon a common pool of SVs at these central synapses (Hua et al., 2010). Due to the spectral separation between cypHer and the GFP-based genetic probe $\mathrm{SpH}$ we could use the two $\mathrm{pH}$-dependent optical reporters in tandem and hence obtain two independent read-outs of presynaptic activity (Fig. 3.19). AP-driven fluorescence transients of aSyt1-cypHer and aVGAT-cypHer-stained boutons exhibited reverse profiles in comparison to $\mathrm{SpH}$ due to their opposite $\mathrm{pH}-$ dependences of fluorescence (Fig. 3.17 and Fig. 3.19). However, the kinetics of endocytosis and reacidification assayed by both methods gave similar estimates showing that this cypHerbased approach is a reliable tool to monitor vesicle recycling at presynaptic boutons.

\subsubsection{Stranded pool of vesicle proteins on the presynaptic membrane}

Upon SV fusion, the vesicle constituents would be expected to mix and get diluted at the presynaptic membrane, which would impose severe constraints on the endocytic machinery to recapture and reform a SV. One possible solution to avoid this large dilution at the surface is 
to maintain a sufficiently sized pool of SV proteins at the presynaptic membrane, which can be readily used by the endocytic machinery. Although the initial attempts to identify vesicle proteins at the plasma membrane, based on immunoassays with intraluminal anti-Syt1 antibodies, were unsuccessful (Kraszewski et al., 1995; Matteoli et al., 1992), later quantitative studies revealed a significant pool of endogenous Syt1 protein at the surface of 'resting' synapses (Fernandez-Alfonso et al., 2006; Willig et al., 2006). In addition, ultrastructural studies also documented the existence of several SV proteins like Syt1 and Syb2 at the synaptic membrane (Taubenblatt et al., 1999). Recent studies using overexpression of pHItagged SV proteins (SpH and Syt1-pHI) have demonstrated a sizeable fraction ( 20-30\%) of these molecules at the surface (Fernandez-Alfonso et al., 2006; Wienisch and Klingauf, 2006). Although overexpression or addition of a $\mathrm{pHI}$ moiety might lead to an imbalance in the targeting of the SV proteins, it has been convincingly shown that the cellular dynamics of the pHI-tagged proteins closely resembles those of the native proteins (Fernandez-Alfonso et al., 2006; Wienisch and Klingauf, 2006).

It was originally proposed that the surface expression of SV proteins arises from to constitutive recycling which may be essential not only during maturation of synapses but also for their functional maintenance (Matteoli et al., 1992). Alternatively, the stranded pool may be a consequence of inefficiencies in the endocytic machinery or ectopic trafficking during synaptogenesis (Ahmari et al., 2000; Sampo et al., 2003). Overexpression does lead to a slight mislocalisation and hence exaggeration of the size of stranded $\mathrm{SpH}$ or Syt1-pHI molecules at the surface, however, the kinetics of retrieval as shown in Fig. $\mathbf{3 . 2 0}$ remain unperturbed and are identical to that of native proteins. Coexpression of Syp with $\mathrm{SpH}$ selectively alleviates the surface pool of $\mathrm{SpH}$ from $30 \%$ to $10 \%$ without affecting the vesicular $\mathrm{SpH}$ fraction or the retrieval time course (Wienisch and Klingauf, 2006). Since Syp interacts with Syb2, it ensures proper targeting of $\mathrm{SpH}$ (Calakos and Scheller, 1994). Therefore, by having similar levels of both proteins, it restores the stochiometry of $\mathrm{SpH}$ and represents a more physiological condition. Amounts of endogenous Syt1 on the surface probed with intraluminal antibodies yielded similar estimates when compared to surface expression of Syt1-pHI (19\% vs $24 \%$ ) indicating little or no mislocalisation (Opazo et al., 2010; Wienisch 
and Klingauf, 2006). Other SV proteins such as Syp-pHI and vGlut-pHI have also been shown to exhibit some surface expression although the surface pool of these proteins is relatively small compared to SpH and Syt1-pHI (Balaji and Ryan, 2007; Granseth et al., 2006). But even the relatively small fraction of surface-stranded endogenous vesicle transporters (VGAT probed with aVGAT-cypHer) is also preferentially retrieved upon exocytosis and appears to have the same capacity as the Syt1 RRetP, indicating that almost all surface-stranded transporter molecules had been efficiently sorted to the RRetP (Fig. 3.20). Thus the existence of a surface pool reduces the dilution of freshly exocytosed SV constituents at the plasma membrane and reduces the load on the endocytic machinery to recapture and resort SV proteins.

\subsubsection{Stimulation-dependent uptake of the readily retrievable pool}

By probing the fate of SV proteins at the bouton membrane, we could directly demonstrate the active and preferential uptake of this surface pool upon stimulated endocytosis thereby uncoupling exo- and endocytosis. As shown in Fig. $\mathbf{3 . 2 0}$ upon stimulation, the cypHer fluorescence transient enabled us for the first time to monitor the dynamics of the SVs derived from a RRetP consisting of endogenous Syt1 or VGAT molecules. To provide direct evidence that this surface pool actively participates in exo-endocytosis, the vesicular cypHer signal from aSyt1-cypHer and aVGAT-cypHer stained boutons was photobleached. A stimulus of 50 APs was then elicited, which is known to deplete the RRP of docked and primed vesicles. The increase in cypHer fluorescence indicates preferential endocytosis of cypHer stained Syt1 and VGAT residing in the external bouton membrane (Fig. 3.20). This preferential recruitment of pre-existing SV constituents upon stimulation remains unperturbed with or without $\mathrm{SpH}$ overexpression, thus validating previous pHI based results (Fernandez-Alfonso et al., 2006; Wienisch and Klingauf, 2006). Although overexpression of some pHI-fusion proteins leads to an excess of surface stranded proteins it has been shown to have no effect on the actual functional size of this pool (Fernandez-Alfonso et al., 2006; Wienisch and Klingauf, 2006). The size of the RRetP could be determined by selective dequenching of surface-bound aSyt1cypHer with a brief application of acidic buffer (Fig. 3.18). Retrieval from the surface pool 
could counterbalance exocytosis for up to 70 APs at $20 \mathrm{~Hz}$ (Fig. 3.18 and Fig. 3.20). We further measured the size of this functional surface pool by monitoring endocytosis of surface Syt1 molecules with a labeling strategy that preferentially stained the surface-resident Syt1 molecules (Fig. 3.21). Thus the RRetP has a capacity similar to the RRP (Fig. 3.20). Moreover, it is interesting to note that even for NT transporters (VGAT), which have been shown to occur in 5-10 copies per SV (Takamori et al., 2006), there is a functional surface pool that is preferentially internalized upon AP triggered exo-endocytosis (Fig. 3.20).

The preferential uptake of SV constituents from a surface repertoire nicely explains the loss of the SV protein complement postfusion and the molecular non-identity of SVs exo- and endocytosed. Although overexpression of $\mathrm{SpH}$ may lead to a significant increase of the total surface pool, using $\mathrm{SpH}$ and cypHer in parallel clearly defines the participation of a functional retrievable surface pool in stimulation-dependent exo-endocytosis. The RRetP could represent preassembled endocytic structures at designated sites near the AZs of these synapses, which are preferentially retrieved during stimulation-dependent exo-endocytosis. Presence of this functional surface pool of presorted endocytic structures might explain a first component of clathrin-mediated endocytosis (Miller and Heuser, 1984). This also explains why the initial endocytic rate remains constant irrespective of the stimulation strength as suggested in other studies (Fernandez-Alfonso and Ryan, 2004; Sankaranarayanan and Ryan, 2000). However, once this RRetP reaches saturation (at $\sim 70 \mathrm{APs}$ ), the rate of endocytosis drops for longer stimuli (Sankaranarayanan and Ryan, 2000) since the endocytic machinery has to invest more energy and time to recapture and resort freshly exocytosed components to the sites of retrieval. Since endocytosis of the RRetP (Fig. 3.20 and Fig. 3.22) itself, however, takes a number of sec, the main advantage of a RRetP might not be so much a gain in speed but rather a gain in the precision of resorting and reclustering to maintain maximum fusion competence of newly endocytosed vesicles. This is in line with the counter-intuitive finding that knock-down or knock-out of major sorting factors like AP-1 and AP-2 subunits result in similar or even faster endocytosis kinetics of a large fraction of retrieved SV proteins (Glyvuk et al., 2010; Kim and Ryan, 2009), probably corresponding to bulk endocytosis. However, the re- 
availability of endocytosed SVs is slowed down and the fusion competence diminished (Glyvuk et al., 2010).

\subsubsection{Spatial organization of the readily retrievable pool}

How is the functional surface pool spatially organized at the presynapse? Previous studies have shown that the endocytic machinery surrounds the active zone and is organised at the periphery of the presynaptic membrane (Brodin and Shupliakov, 2006; Roos and Kelly, 1999; Teng et al., 1999; Teng and Wilkinson, 2000). In collaboration with the laboratory of Prof. Stefan W. Hell at the Dept. of Nanobiophotonics (Max-Planck Inst. for Biophysical Chemistry), the spatial organization of the Syt1 surface pool at hippocampal synapses was investigated by my colleague Cora S. Thiel using dual-color isotropic stimulated emission depletion (isoSTED) (Schmidt et al., 2008) nanoscopy. After surface Syt1 staining, cells were fixed, permeabilized and labeled with the cytomatrix of active zone (CAZ) marker, RIM1/2 and the post-synaptic density (PSD) scaffolding protein, Homer1. In the three-dimensional reconstruction of hippocampal en passant boutons from isoSTED image stacks (Fig. 4.2), distinct Syt1 patches were found in the peri-active zone of about $500 \mathrm{~nm}$ around the active and PSD zones, respectively. This pattern resembled a doughnut-like arrangement of Syt1 patches at around the AZ (RIM1/2) and PSD complexes (Homer1). This is in agreement with previous observations of endocytic components which were found to be enriched at the peri-active zone, generally identified as the site of endocytosis which revealed several preassembled patches of clathrin coats from which new vesicles could bud off (Brodin and Shupliakov, 2006; Roos and Kelly, 1999; Teng et al., 1999; Teng and Wilkinson, 2000). 
(a)

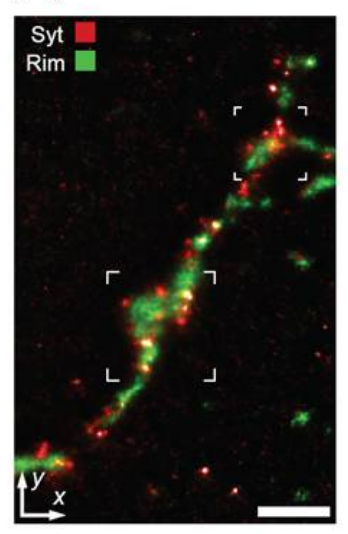

(b)

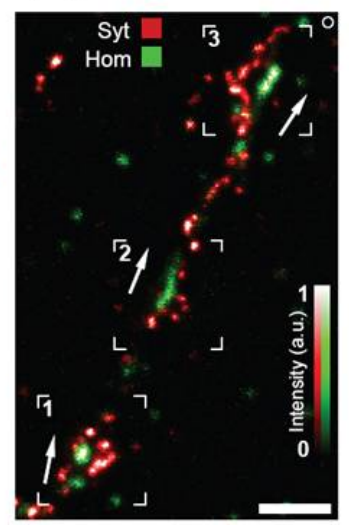

(c)

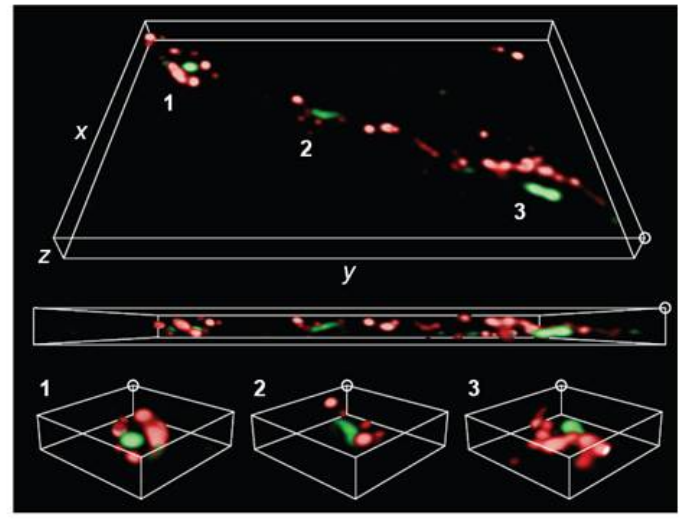

Figure 4.2: Spatial organization of the readily retrievable pool.

3D dual-color isoSTED nanoscopy of cultured hippocampal boutons showing the localization of surfacestranded Syt1 (red) at synapses identified by the CAZ marker, RIM1/2 (green) and the PSD marker, Homer1 (green) respectively.

(a \& b) z-Projection of a 3D image stack of Syt1 at synapses labeled by RIM1/2 (a) and by Homer1 (b), with synapses at ROI numbered 1 to 3 . Arrows mark the local orientation of the axon.

(c) Perspective views of volume rendered data. The Syt1 labeling reveals preassembled patches, which are localized in the periphery of the active zone, i.e. typically within $500 \mathrm{~nm}$ distance from Homer1 patches.

Courtesy: C. S. Thiel, R. Schmidt and A. Egner.

The centre-surround arrangement of exo- and endocytic zones suggests that newly added SV constituents migrate laterally at the plasma membrane from the release sites to the endocytic sites for subsequent retrieval. Since there is no barrier for diffusional exchange, SV components post fusion could laterally disperse within the bouton membrane and may be recaptured and reclustered at the peri-active zone by adaptor proteins, thus repopulating the RRetP with fresh SV constituents. This way SV material could be rapidly and efficiently cleared from the release site and then retained at the RRetP for compensatory endocytosis. Thus, the preassembled patches of Syt1 at the peri-active zone, as seen in the isoSTED images (Fig. 4.2), are fully consistent with fast recapturing of exocytosed SV proteins in the RRetP. In fact clusters of Syt1 on the presynaptic membrane were previously detected by 
STED microscopy in a separate study (Willig et al., 2006), which concluded that the SV constituents do not disperse but remain clustered post fusion although the degree of clustering was too small (about two-fold) to prevent diffusional dispersion, and furthermore the measurements on fixed neurons provide only a snapshot and do not reveal dynamics.

In order to sustain release, freshly exocytosed SV constituents need to be effectively removed from the release site, such that its intricate molecular organization and integrity is not disturbed for the next round of fusion. Clearance of release sites may indeed be the ratelimiting step for docking, priming and fusion of new SVs, since accumulation of SV proteins could jam the release site and render them refractory to further release. Previous studies have shown that acute perturbation of endocytic proteins, such as dynamin, led to a delayed SV replenishment and an enhanced short-term depression (Hosoi et al., 2009; Kawasaki et al., 2000). Since the number of SVs in the synapse remained unaltered, these short-term effects could not be explained by the unavailability of SVs for fusion. Therefore, an intermediate step in exo-endocytic coupling, most likely the clearance of release sites was thought to be disrupted (Hosoi et al., 2009). Post fusion release sites could be cleared by two different modes (Fig. 4.3). In the first mode, freshly exocytosed SV proteins remain clustered and migrate in membrane patches towards the rim of the active zone by directed diffusion (Fig. 4.3). However, clustering of SV proteins in a patch might actually jam release sites and block clearance. In the second more likely scenario, the SV proteins initially disperse and decluster after fusion followed by fast reclustering mediated by endocytic adaptor proteins, such as AP-2 and stonin 2 (Diril et al., 2006; Kim and Ryan, 2009), enriched near the AZ (Fig. 4.3). Thus the rate of diffusional dispersion of SV constituents after fusion, away from the release site, would determine how fast the release site is cleared and hence available for the next release event. 
(a)
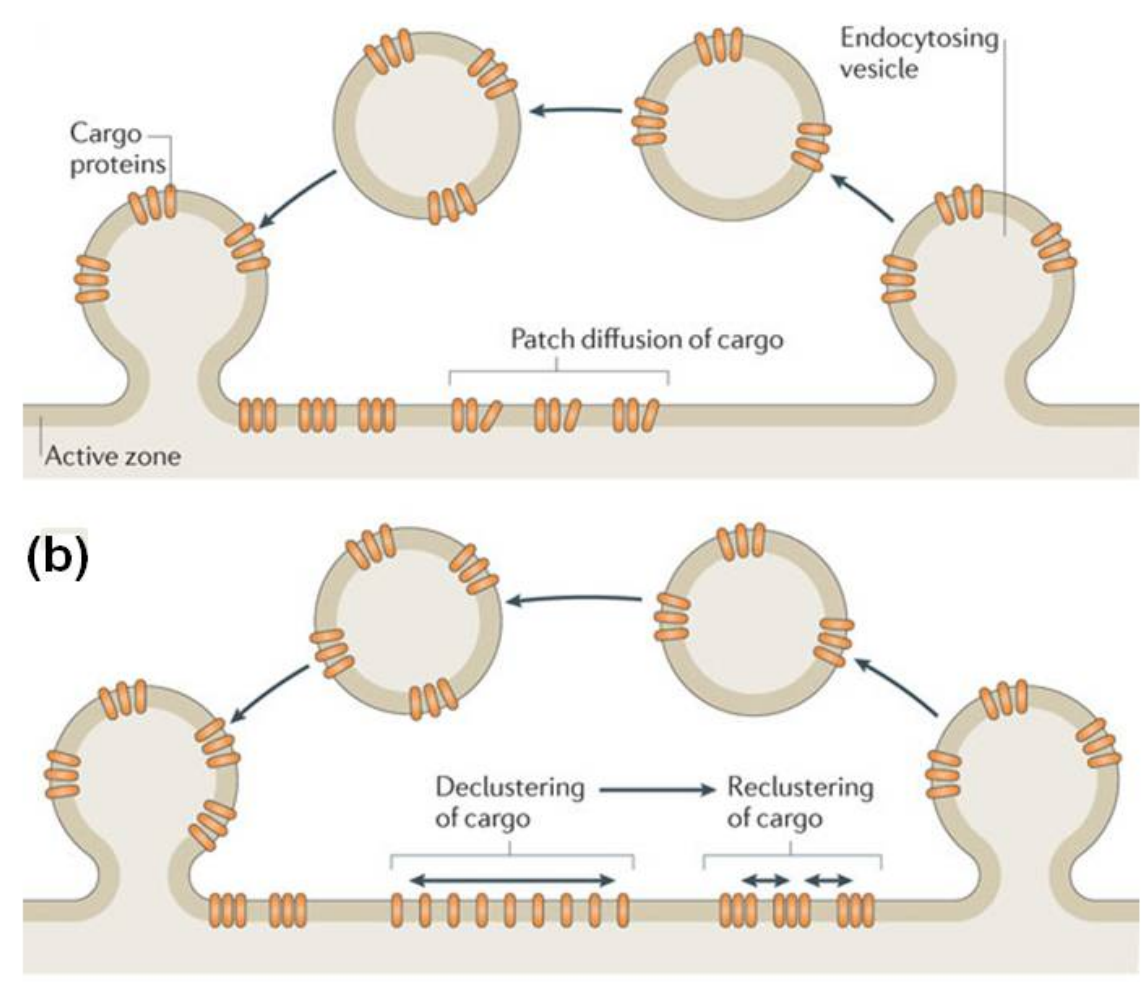

Figure 4.3: Models of release site clearance.

(a) SV cargo proteins remain clustered after exocytosis and diffuse as a unit in raft-like patches towards the endocytic sites thereby coupling exo-and endocytosis.

(b) SV disintegrates and its constituents disperse upon fusion and undergo rapid diffusion. This is followed by recapturing and reclustering by adaptor proteins at the periactive zone for compensatory endocytosis.

Taken from (Haucke et al., 2011).

\subsubsection{Possible mechanisms of exo-endocytic coupling}

The regulatory mechanisms coupling exo- and endocytosis are not well understood. Exocytosis itself could be a trigger for compensatory endocytosis, where fused membrane bound SV proteins signal their retrieval by interacting with specific adaptor proteins. Indeed neurons lacking SV proteins required for exocytosis, such as Syb2 and Syt1, show striking deficits in endocytosis (Deak et al., 2004; Nicholson-Tomishima and Ryan, 2004). Another 
regulatory factor coupling exo- and endocytosis could be local intracellular $\mathrm{Ca}^{2+}$. Interestingly in synaptic terminals, where exocytosis was blocked with BoNT/A, influx of $\mathrm{Ca}^{2+}$ upon membrane depolarization was sufficient to trigger endocytosis (Neale et al., 1999). In three recent studies, the importance of $\mathrm{Ca}^{2+}$ for coupling of exo- and endocyosis has been emphasized (Hosoi et al., 2009; Wu et al., 2009; Yao et al., 2009). On the contrary, upon $\mathrm{Ca}^{2+}$-independent release induced by hypertonic sucrose, compensatory endocytosis was found to be intact (Pyle et al., 2000). Thus, it is unclear whether $\mathrm{Ca}^{2+}$ really mediates or merely modulates exo-endocytic coupling. Moreover, it has been proposed that $\mathrm{Ca}^{2+}$ elevation during stimulation triggers a phosphorylation cascade of several accessory proteins which are essential for endocytosis (Cousin and Robinson, 2001). Work in Drosophila neuromuscular junctions suggests that there might be a vesicular calcium channel, flower, that once incorporated into the plasma membrane during SV fusion would allow $\mathrm{Ca}^{2+}$ entry at the site of endocytosis and thus might trigger the retrieval of SVs from the RRetP (Yao et al., 2009). However, the detailed mechanism that couples exocytosis from the RRP and compensatory endocytosis from the RRetP and the molecular underpinnings remain to be elucidated. 


\section{SUMMARY}

In this study, I have utilized pH-switchable reporters, both genetically encoded GFP-based pHI and exogenous cypHer5E-based probes, to first estimate the minimum copy numbers of fusogenic molecules required for SV exocytosis, and secondly to investigate the spatiotemporal coupling of exo- and endocytosis. pHI-fusion chimeras enabled single SV and single molecule optical resolution, which was crucial for determining protein numbers required for exocytosis. On the other hand, the cypHer probe enabled tracking of endogenous SV proteins during endocytosis with kinetics identical to pHI-fusion chimeras, thereby providing a complementary tool to study SV recycling.

Fast exocytosis during synaptic transmission in conventional bouton-type synapses is the most studied fusion reaction. However, the exact number of SNARE complexes minimally needed for fusion during synaptic transmission is controversially debated. This study presents the first successful attempt to optically resolve single SNARE molecules mediating fusion and then counting the minimum number of these molecules ('nano proteomics') required to drive fusion during fast $\mathrm{Ca}^{2+}$-triggered exocytosis in a physiological setting. Previous studies have suggested a wide range of SNARE complex estimates (from 1 to 15), largely based on indirect determinations. High-resolution optical recordings of single SV fusion, using v-SNARE molecules coupled to $\mathrm{pHI}(\mathrm{SpH})$, followed by quantitative single $\mathrm{pHI}$ molecule calibration gave a direct estimate of the exact number of $\mathrm{SpH}$ molecules per $\mathrm{SV}$. This number was quite low with a sizeable fraction of SVs containing a single copy of $\mathrm{SpH}$. When overexpressed on a null background (in absence of the endogenous complement of Syb2) one copy of SpH was unable to mediate evoked fusion. Thus, a minimum of two $\mathrm{SpH}$ copies and hence two SNARE complexes are necessary and sufficient for SV fusion during fast synaptic transmission. This is a major revision of current estimates, and asks for a reconsideration of the current popular models for membrane fusion that invoke multiple SNARE complexes in a ring-shaped arrangement. Furthermore, the present finding has important implications for the mechanisms underlying SV docking and priming. 
The above approach was also applied to quantify the number of Syt1-pHI molecules required to sense $\mathrm{Ca}^{2+}$ and evoke SV fusion. The amplitude histograms indicate that a single molecule of Syt1-pHI is sufficient for SV fusion. However, the results need to be complemented by other kinetic and biochemical measurements.

In the second part of this study, I together with my colleague Yunfeng Hua introduced a novel $\mathrm{pH}$-sensitive exogenous optical tracer; a cyanine dye variant (CypHer5E) coupled to antibodies against specific endogenous SV proteins Syt1 and VGAT, to reliably monitor exoendocytosis during SV recycling in hippocampal synapses in real time. This new technique provided an alternative and complementary tool to monitor the spatio-temporal dynamics of SV protein recycling and to study mechanisms of exo- and endocytosis. This approach eliminates the need of overexpression - and possible artifacts that come with it - like for pHIfusion constructs or of using non-specific membrane markers like FM dyes. Additionally, by monitoring the recycling of endogenous SV proteins labeled with this new $\mathrm{pH}$ sensor, we visualized for the first time the spatial and temporal kinetics of a functional surface pool of SV proteins (RRetP) from the presynaptic membrane upon stimulation. Once the RRetP, which has a size equivalent to that of the RRP, is depleted, it is repopulated by freshly exocytosed SV constituents. This was corroborated by isoSTED microscopy conducted in parallel by my colleague Cora S. Thiel in collaboration with the laboratory of Prof. Stefan W. Hell (Dept. of Nanobiophotonics, Max-Planck Inst. for Biophysical Chemistry, Göttingen), where they measured the spatial distribution of the surface pool of SV constituents in hippocampal boutons. SV proteins at the presynaptic membrane were largely concentrated at the periactive zone close to the periphery of the bouton. These represent putative sites of endocytosis from where preassembled SV constituents might be readily retrieved during activity thereby enabling small CNS synapses to maintain a steady-state rate of synaptic transmission. 
(a)

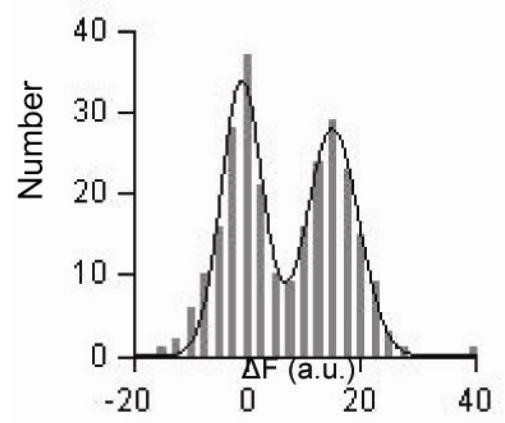

$\sigma_{0}=5.15$

$\sigma_{\mathrm{k} 1}=6.43$ (b)

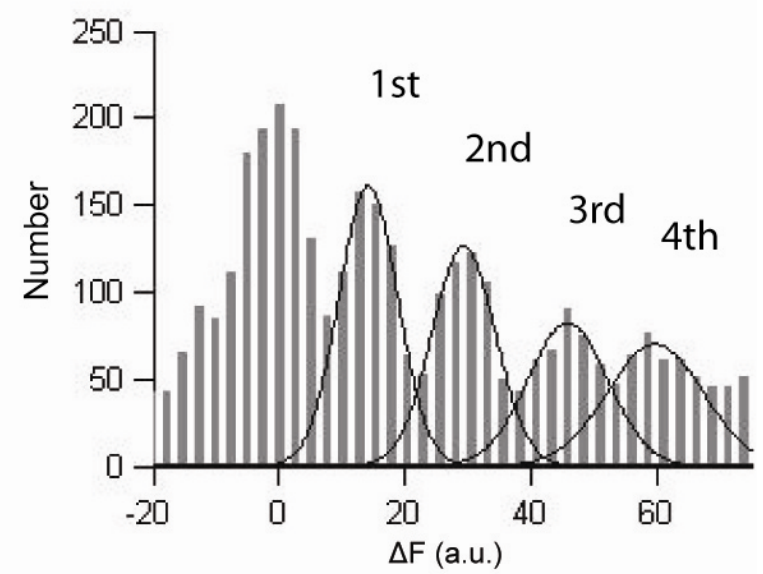

From eqn (iii) $\sigma_{q}=3.84$

(c)

\begin{tabular}{|c|c|c|c|}
\hline $\begin{array}{c}\text { Peak no.(non- } \\
\text { zero peaks) }\end{array}$ & $\begin{array}{c}\text { Predicted } \sigma_{k} \\
\text { from Fig (a) }\end{array}$ & $\begin{array}{c}\sigma_{k} \text { from Gaussian } \\
\text { fitting (Fig b) }\end{array}$ & $\begin{array}{c}\mu_{k} \text { from Gaussian } \\
\text { fitting (Fig b) }\end{array}$ \\
\hline $1^{\text {st }}$ & 6.43 & 6.61 & $14.21 \pm 1.05$ \\
\hline $2^{\text {nd }}$ & 7.48 & 7.15 & $29.35 \pm 1.87$ \\
\hline $3^{\text {rd }}$ & 8.41 & 9.003 & $45.9 \pm 1.67$ \\
\hline $4^{\text {th }}$ & 9.24 & 10.77 & $59.79 \pm 2.1$ \\
\hline
\end{tabular}

Figure S1: Comparison of standard deviations of $\Delta \mathrm{F}$ distributions from single molecule and single AP experiments.

(a) Amplitude distribution of single molecule bleaching steps from Fig 3.5. The quantal standard deviation $\sigma_{q}$ was estimated from eqn (iii).

(b) Amplitude distribution of single AP fluorescence response from Fig. 3.9 fitted with individual gaussian functions for each of the non-zero peaks.

(c) Table comparing the calculated $\sigma_{k}$ from (a) and eqn (iii; Material and Methods) with those predicted by (b). There is a marginal increase in the $\sigma_{k}$ values. The predicated $\mu_{k}$ values increase in multiples of the quantal fluorescence (mean of the 1st peak) i.e. $14.21 \pm 1.05$ units fully consistent with the multigaussian estimates. 
(a)

\section{Syp-pHI}

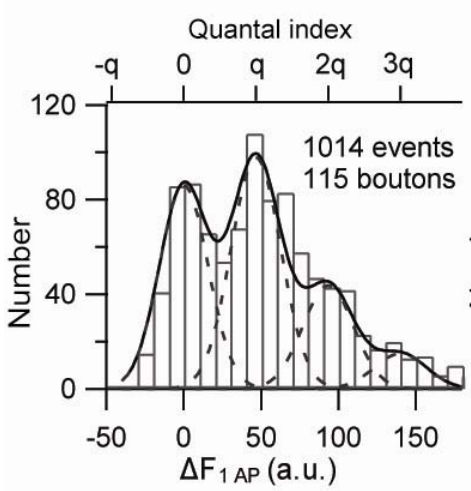

(b)

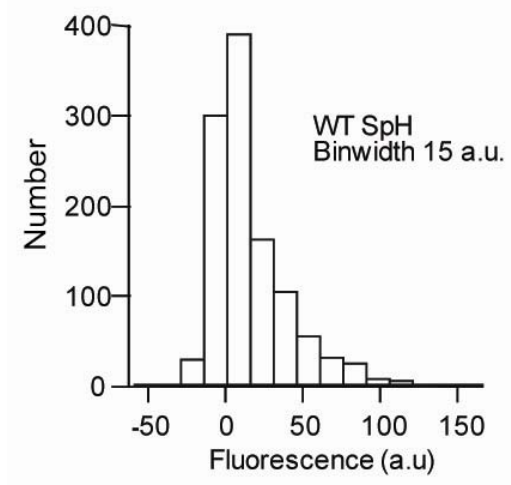

vGlut-pHI

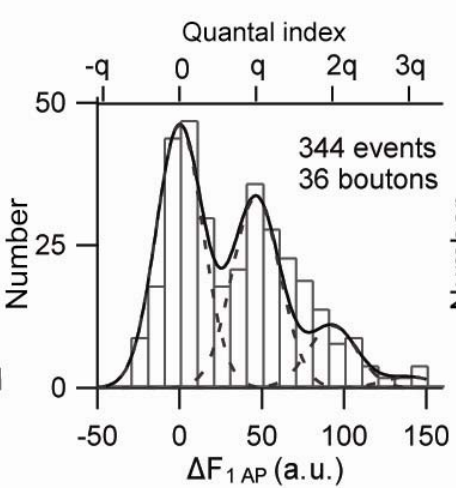

$\mathrm{SpH}$

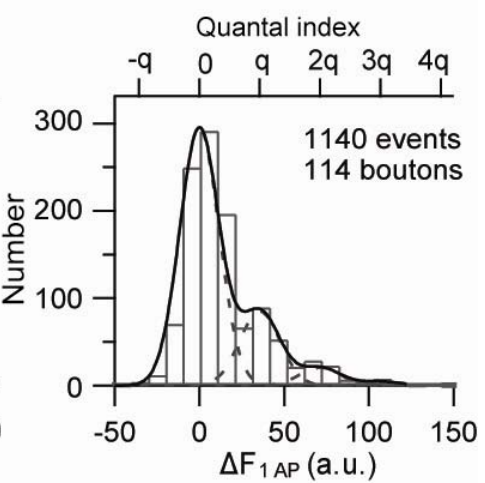

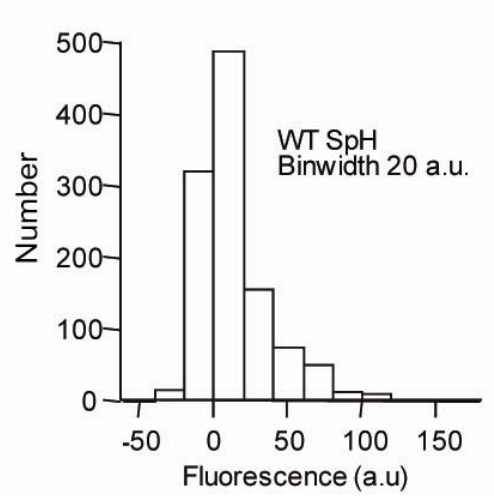

Figure S2: An average of 2-3 copies of pHI-tagged proteins are targeted per SV.

(a) $\Delta \mathrm{F}$ Histograms from Syp-pHI, vGlut-pHI, and $\mathrm{SpH}$ transfected boutons replotted with a bin width of 10 a.u. The solid and dashed lines are the overall and individual fits to multiple Gaussians of fixed quantal size $q \pm$ s.d. The estimated q for Syp-pHI is $46.8 \pm 1.44$ a.u (adjusted $R^{2}=0.91$ ), for vGlut-pHI $46.5 \pm 1.95$ a.u (adjusted $R^{2}=0.94$ ), and for $\mathrm{SpH} 35.6 \pm 3.85$ a.u (adjusted $R^{2}=0.98$ ). Comparison with single pHI molecule intensity (Fig. 3.5) reveals that on average about 2-3 copies of pHI-tagged proteins are incorporated per SV.

(b) Coarser binning of $\mathrm{SpH} \Delta \mathrm{F}$ distributions (from (a) with bin width $>10$ a.u.) eliminates any peaks that are visible in the histograms with a binwidth of 10 and 2.5 a.u. 
Ahmari, S.E., Buchanan, J., and Smith, S.J. (2000). Assembly of presynaptic active zones from cytoplasmic transport packets. Nat Neurosci 3, 445-451.

Alvarez de Toledo, G., Fernandez-Chacon, R., and Fernandez, J.M. (1993). Release of secretory products during transient vesicle fusion. Nature $363,554-558$.

Antonin, W., Holroyd, C., Fasshauer, D., Pabst, S., Von Mollard, G.F., and Jahn, R. (2000). A SNARE complex mediating fusion of late endosomes defines conserved properties of SNARE structure and function. EMBO J 19, 6453-6464.

Aravanis, A.M., Pyle, J.L., and Tsien, R.W. (2003). Single synaptic vesicles fusing transiently and successively without loss of identity. Nature 423, 643-647.

Ashby, M.C., Ibaraki, K., and Henley, J.M. (2004). It's green outside: tracking cell surface proteins with pH-sensitive GFP. Trends Neurosci 27, 257-261.

Axelrod, D. (2003). Total internal reflection fluorescence microscopy in cell biology. Methods Enzymol 361, 1-33.

Balaji, J., and Ryan, T.A. (2007). Single-vesicle imaging reveals that synaptic vesicle exocytosis and endocytosis are coupled by a single stochastic mode. Proc Natl Acad Sci U S A 104, 20576-20581.

Bock, J.B., Matern, H.T., Peden, A.A., and Scheller, R.H. (2001). A genomic perspective on membrane compartment organization. Nature 409, 839-841.

Bollmann, J.H., Sakmann, B., and Borst, J.G. (2000). Calcium sensitivity of glutamate release in a calyxtype terminal. Science 289, 953-957.

Borisovska, M., Zhao, Y., Tsytsyura, Y., Glyvuk, N., Takamori, S., Matti, U., Rettig, J., Sudhof, T., and Bruns, D. (2005). v-SNAREs control exocytosis of vesicles from priming to fusion. EMBO J 24, 21142126.

Bowen, M.E., Weninger, K., Brunger, A.T., and Chu, S. (2004). Single molecule observation of liposomebilayer fusion thermally induced by soluble $\mathrm{N}$-ethyl maleimide sensitive-factor attachment protein receptors (SNAREs). Biophys J 87, 3569-3584.

Bowser, D.N., and Khakh, B.S. (2007). Two forms of single-vesicle astrocyte exocytosis imaged with total internal reflection fluorescence microscopy. Proc Natl Acad Sci U S A 104, 4212-4217.

Brock, R., Hamelers, I.H., and Jovin, T.M. (1999). Comparison of fixation protocols for adherent cultured cells applied to a GFP fusion protein of the epidermal growth factor receptor. Cytometry 35, 353-362.

Brodin, L., Low, P., and Shupliakov, O. (2000). Sequential steps in clathrin-mediated synaptic vesicle endocytosis. Curr Opin Neurobiol 10, 312-320.

Brodin, L., and Shupliakov, O. (2006). Giant reticulospinal synapse in lamprey: molecular links between active and periactive zones. Cell Tissue Res 326, 301-310.

Brodsky, F.M., Chen, C.Y., Knuehl, C., Towler, M.C., and Wakeham, D.E. (2001). Biological basket weaving: formation and function of clathrin-coated vesicles. Annu Rev Cell Dev Biol 17, 517-568.

Brose, N., Rosenmund, C., and Rettig, J. (2000). Regulation of transmitter release by Unc-13 and its homologues. Curr Opin Neurobiol 10, 303-311.

Calakos, N., and Scheller, R.H. (1994). Vesicle-associated membrane protein and synaptophysin are associated on the synaptic vesicle. J Biol Chem 269, 24534-24537.

Ceccarelli, B., Hurlbut, W.P., and Mauro, A. (1972). Depletion of vesicles from frog neuromuscular junctions by prolonged tetanic stimulation. J Cell Biol 54, 30-38.

Ceccarelli, B., Hurlbut, W.P., and Mauro, A. (1973). Turnover of transmitter and synaptic vesicles at the frog neuromuscular junction. J Cell Biol 57, 499-524.

Chalfie, M., Tu, Y., Euskirchen, G., Ward, W.W., and Prasher, D.C. (1994). Green fluorescent protein as a marker for gene expression. Science 263, 802-805.

Chapman, E.R. (2008). How does synaptotagmin trigger neurotransmitter release? Annu Rev Biochem 77, 615-641. 
Chen, Y.A., Scales, S.J., and Scheller, R.H. (2001). Sequential SNARE assembly underlies priming and triggering of exocytosis. Neuron $30,161-170$.

Chiu, C.S., Jensen, K., Sokolova, I., Wang, D., Li, M., Deshpande, P., Davidson, N., Mody, I., Quick, M.W., Quake, S.R., et al. (2002). Number, density, and surface/cytoplasmic distribution of GABA transporters at presynaptic structures of knock-in mice carrying GABA transporter subtype 1-green fluorescent protein fusions. J Neurosci 22, 10251-10266.

Chiu, C.S., Kartalov, E., Unger, M., Quake, S., and Lester, H.A. (2001). Single-molecule measurements calibrate green fluorescent protein surface densities on transparent beads for use with 'knock-in' animals and other expression systems. J Neurosci Methods 105, 55-63.

Choi, S., Klingauf, J., and Tsien, R.W. (2000). Postfusional regulation of cleft glutamate concentration during LTP at 'silent synapses'. Nat Neurosci 3, 330-336.

Chung, C., Barylko, B., Leitz, J., Liu, X., and Kavalali, E.T. (2010). Acute dynamin inhibition dissects synaptic vesicle recycling pathways that drive spontaneous and evoked neurotransmission. J Neurosci 30, 1363-1376.

Cohen, F.S., and Melikyan, G.B. (2004). The energetics of membrane fusion from binding, through hemifusion, pore formation, and pore enlargement. J Membr Biol 199, 1-14.

Cousin, M.A., and Robinson, P.J. (2001). The dephosphins: dephosphorylation by calcineurin triggers synaptic vesicle endocytosis. Trends Neurosci 24, 659-665.

Couteaux, R., and Pecot-Dechavassine, M. (1970). [Synaptic vesicles and pouches at the level of "active zones" of the neuromuscular junction]. C R Acad Sci Hebd Seances Acad Sci D 271, 2346-2349.

Cremona, O., and De Camilli, P. (1997). Synaptic vesicle endocytosis. Curr Opin Neurobiol 7, 323-330.

Cremona, O., Di Paolo, G., Wenk, M.R., Luthi, A., Kim, W.T., Takei, K., Daniell, L., Nemoto, Y., Shears, S.B., Flavell, R.A., et al. (1999). Essential role of phosphoinositide metabolism in synaptic vesicle recycling. Cell 99, 179-188.

Danglot, L., and Galli, T. (2007). What is the function of neuronal AP-3? Biol Cell 99, 349-361.

Danieli, T., Pelletier, S.L., Henis, Y.I., and White, J.M. (1996). Membrane fusion mediated by the influenza virus hemagglutinin requires the concerted action of at least three hemagglutinin trimers. J Cell Biol 133, 559-569.

Davis, G.W. (2006). Homeostatic control of neural activity: from phenomenology to molecular design. Annu Rev Neurosci 29, 307-323.

de Lange, R.P., de Roos, A.D., and Borst, J.G. (2003). Two modes of vesicle recycling in the rat calyx of Held. J Neurosci 23, 10164-10173.

de Wit, H., Cornelisse, L.N., Toonen, R.F., and Verhage, M. (2006). Docking of secretory vesicles is syntaxin dependent. PLoS One 1, e126.

de Wit, H., Walter, A.M., Milosevic, I., Gulyas-Kovacs, A., Riedel, D., Sorensen, J.B., and Verhage, M. (2009). Synaptotagmin-1 Docks Secretory Vesicles to Syntaxin-1/SNAP-25 Acceptor Complexes. Cell.

Deak, F., Schoch, S., Liu, X., Sudhof, T.C., and Kavalali, E.T. (2004). Synaptobrevin is essential for fast synaptic-vesicle endocytosis. Nat Cell Biol 6, 1102-1108.

Deak, F., Shin, O.H., Kavalali, E.T., and Sudhof, T.C. (2006). Structural determinants of synaptobrevin 2 function in synaptic vesicle fusion. J Neurosci $26,6668-6676$.

Dickson, R.M., Cubitt, A.B., Tsien, R.Y., and Moerner, W.E. (1997). On/off blinking and switching behaviour of single molecules of green fluorescent protein. Nature 388, 355-358.

Diril, M.K., Wienisch, M., Jung, N., Klingauf, J., and Haucke, V. (2006). Stonin 2 is an AP-2-dependent endocytic sorting adaptor for synaptotagmin internalization and recycling. Dev Cell 10, 233-244.

Dittman, J., and Ryan, T.A. (2009). Molecular circuitry of endocytosis at nerve terminals. Annu Rev Cell Dev Biol 25, 133-160.

Dittman, J.S., and Kaplan, J.M. (2006). Factors regulating the abundance and localization of synaptobrevin in the plasma membrane. Proc Natl Acad Sci U S A 103, 11399-11404.

Dodge, F.A., Jr., and Rahamimoff, R. (1967). Co-operative action a calcium ions in transmitter release at the neuromuscular junction. J Physiol 193, 419-432. 
Domanska, M.K., Kiessling, V., Stein, A., Fasshauer, D., and Tamm, L.K. (2009). Single vesicle millisecond fusion kinetics reveals number of SNARE complexes optimal for fast SNARE-mediated membrane fusion. J Biol Chem.

Donovan, F.M., Pike, C.J., Cotman, C.W., and Cunningham, D.D. (1997). Thrombin induces apoptosis in cultured neurons and astrocytes via a pathway requiring tyrosine kinase and RhoA activities. J Neurosci $17,5316-5326$.

Dulubova, I., Sugita, S., Hill, S., Hosaka, M., Fernandez, I., Sudhof, T.C., and Rizo, J. (1999). A conformational switch in syntaxin during exocytosis: role of munc18. EMBO J 18, 4372-4382.

Eisenberg, E., and Greene, L.E. (2007). Multiple roles of auxilin and hsc70 in clathrin-mediated endocytosis. Traffic 8, 640-646.

Eliasson, L., Renstrom, E., Ding, W.G., Proks, P., and Rorsman, P. (1997). Rapid ATP-dependent priming of secretory granules precedes $\mathrm{Ca}(2+)$-induced exocytosis in mouse pancreatic B-cells. J Physiol 503 ( Pt 2), 399-412.

Fasshauer, D., Eliason, W.K., Brunger, A.T., and Jahn, R. (1998a). Identification of a minimal core of the synaptic SNARE complex sufficient for reversible assembly and disassembly. Biochemistry $37,10354-$ 10362.

Fasshauer, D., and Margittai, M. (2004). A transient N-terminal interaction of SNAP-25 and syntaxin nucleates SNARE assembly. J Biol Chem 279, 7613-7621.

Fasshauer, D., Sutton, R.B., Brunger, A.T., and Jahn, R. (1998b). Conserved structural features of the synaptic fusion complex: SNARE proteins reclassified as Q- and R-SNAREs. Proc Natl Acad Sci U S A 95, 15781-15786.

Fdez, E., Jowitt, T.A., Wang, M.C., Rajebhosale, M., Foster, K., Bella, J., Baldock, C., Woodman, P.G., and Hilfiker, S. (2008). A role for soluble N-ethylmaleimide-sensitive factor attachment protein receptor complex dimerization during neurosecretion. Mol Biol Cell 19, 3379-3389.

Ferguson, S.M., Brasnjo, G., Hayashi, M., Wolfel, M., Collesi, C., Giovedi, S., Raimondi, A., Gong, L.W., Ariel, P., Paradise, S., et al. (2007). A selective activity-dependent requirement for dynamin 1 in synaptic vesicle endocytosis. Science 316, 570-574.

Fernandez-Alfonso, T., Kwan, R., and Ryan, T.A. (2006). Synaptic vesicles interchange their membrane proteins with a large surface reservoir during recycling. Neuron $51,179-186$.

Fernandez-Alfonso, T., and Ryan, T.A. (2004). The kinetics of synaptic vesicle pool depletion at CNS synaptic terminals. Neuron 41, 943-953.

Fernandez-Chacon, R., Konigstorfer, A., Gerber, S.H., Garcia, J., Matos, M.F., Stevens, C.F., Brose, N., Rizo, J., Rosenmund, C., and Sudhof, T.C. (2001). Synaptotagmin I functions as a calcium regulator of release probability. Nature 410, 41-49.

Fiebig, K.M., Rice, L.M., Pollock, E., and Brunger, A.T. (1999). Folding intermediates of SNARE complex assembly. Nat Struct Biol 6, 117-123.

Fredj, N.B., and Burrone, J. (2009). A resting pool of vesicles is responsible for spontaneous vesicle fusion at the synapse. Nat Neurosci 12, 751-758.

Gad, H., Low, P., Zotova, E., Brodin, L., and Shupliakov, O. (1998). Dissociation between Ca2+-triggered synaptic vesicle exocytosis and clathrin-mediated endocytosis at a central synapse. Neuron 21, 607-616.

Gandhi, S.P., and Stevens, C.F. (2003). Three modes of synaptic vesicular recycling revealed by singlevesicle imaging. Nature 423, 607-613.

Geppert, M., Goda, Y., Hammer, R.E., Li, C., Rosahl, T.W., Stevens, C.F., and Sudhof, T.C. (1994). Synaptotagmin I: a major Ca2+ sensor for transmitter release at a central synapse. Cell 79, 717-727.

Gestaut, D.R., Graczyk, B., Cooper, J., Widlund, P.O., Zelter, A., Wordeman, L., Asbury, C.L., and Davis, T.N. (2008). Phosphoregulation and depolymerization-driven movement of the Dam1 complex do not require ring formation. Nat Cell Biol 10, 407-414.

Gibbons, D.L., Vaney, M.C., Roussel, A., Vigouroux, A., Reilly, B., Lepault, J., Kielian, M., and Rey, F.A. (2004). Conformational change and protein-protein interactions of the fusion protein of Semliki Forest virus. Nature $427,320-325$.

Glyvuk, N., Tsytsyura, Y., Geumann, C., D'Hooge, R., Huve, J., Kratzke, M., Baltes, J., Boning, D., Klingauf, J., and Schu, P. (2010). AP-1/sigma1B-adaptin mediates endosomal synaptic vesicle recycling, learning and memory. EMBO J 29, 1318-1330. 
Granseth, B., Odermatt, B., Royle, S.J., and Lagnado, L. (2006). Clathrin-mediated endocytosis is the dominant mechanism of vesicle retrieval at hippocampal synapses. Neuron 51, 773-786.

Groemer, T.W., and Klingauf, J. (2007). Synaptic vesicles recycling spontaneously and during activity belong to the same vesicle pool. Nat Neurosci 10, 145-147.

Groffen, A.J., Martens, S., Diez Arazola, R., Cornelisse, L.N., Lozovaya, N., de Jong, A.P., Goriounova, N.A., Habets, R.L., Takai, Y., Borst, J.G., et al. (2010). Doc2b is a high-affinity Ca2+ sensor for spontaneous neurotransmitter release. Science 327, 1614-1618.

Gundelfinger, E.D., Kessels, M.M., and Qualmann, B. (2003). Temporal and spatial coordination of exocytosis and endocytosis. Nat Rev Mol Cell Biol 4, 127-139.

Guzman, R.E., Schwarz, Y.N., Retting, J., and Bruns, D. (2010). SNARE force synchronizes synaptic vesicle fusion and controls the kinetics of quantal synaptic transmission. J Neurosci in press.

Hack, N.J., Billups, B., Guthrie, P.B., Rogers, J.H., Muir, E.M., Parks, T.N., and Kater, S.B. (2000). Green fluorescent protein as a quantitative tool. J Neurosci Methods 95, 177-184.

Hammarlund, M., Palfreyman, M.T., Watanabe, S., Olsen, S., and Jorgensen, E.M. (2007). Open syntaxin docks synaptic vesicles. PLoS Biol 5, e198.

Han, X., Wang, C.T., Bai, J., Chapman, E.R., and Jackson, M.B. (2004). Transmembrane segments of syntaxin line the fusion pore of Ca2+-triggered exocytosis. Science 304, 289-292.

Hanson, P.I., Roth, R., Morisaki, H., Jahn, R., and Heuser, J.E. (1997). Structure and conformational changes in NSF and its membrane receptor complexes visualized by quick-freeze/deep-etch electron microscopy. Cell 90, 523-535.

Harata, N.C., Choi, S., Pyle, J.L., Aravanis, A.M., and Tsien, R.W. (2006). Frequency-dependent kinetics and prevalence of kiss-and-run and reuse at hippocampal synapses studied with novel quenching methods. Neuron 49, 243-256.

Harlow, M., Ress, D., Koster, A., Marshall, R.M., Schwarz, M., and McMahan, U.J. (1998). Dissection of active zones at the neuromuscular junction by EM tomography. J Physiol Paris 92, 75-78.

Harlow, M.L., Ress, D., Stoschek, A., Marshall, R.M., and McMahan, U.J. (2001). The architecture of active zone material at the frog's neuromuscular junction. Nature 409, 479-484.

Harris, T.W., Hartwieg, E., Horvitz, H.R., and Jorgensen, E.M. (2000). Mutations in synaptojanin disrupt synaptic vesicle recycling. J Cell Biol 150, 589-600.

Haucke, V., Neher, E., and Sigrist, S.J. (2011). Protein scaffolds in the coupling of synaptic exocytosis and endocytosis. Nat Rev Neurosci 12, 127-138.

Hayashi, T., McMahon, H., Yamasaki, S., Binz, T., Hata, Y., Sudhof, T.C., and Niemann, H. (1994). Synaptic vesicle membrane fusion complex: action of clostridial neurotoxins on assembly. EMBO J 13, 5051-5061.

He, L., and Wu, L.G. (2007). The debate on the kiss-and-run fusion at synapses. Trends Neurosci 30, 447-455.

He, L., Wu, X.S., Mohan, R., and Wu, L.G. (2006). Two modes of fusion pore opening revealed by cellattached recordings at a synapse. Nature $444,102-105$.

Heerssen, H., Fetter, R.D., and Davis, G.W. (2008). Clathrin dependence of synaptic-vesicle formation at the Drosophila neuromuscular junction. Curr Biol 18, 401-409.

Heuser, J.E., and Reese, T.S. (1973). Evidence for recycling of synaptic vesicle membrane during transmitter release at the frog neuromuscular junction. J Cell Biol 57, 315-344.

Holt, M., Cooke, A., Wu, M.M., and Lagnado, L. (2003). Bulk membrane retrieval in the synaptic terminal of retinal bipolar cells. J Neurosci 23, 1329-1339.

Hosoi, N., Holt, M., and Sakaba, T. (2009). Calcium dependence of exo- and endocytotic coupling at a glutamatergic synapse. Neuron 63, 216-229.

Hu, C., Ahmed, M., Melia, T.J., Sollner, T.H., Mayer, T., and Rothman, J.E. (2003). Fusion of cells by flipped SNAREs. Science 300, 1745-1749.

Hua, Y., and Scheller, R.H. (2001). Three SNARE complexes cooperate to mediate membrane fusion. Proc Natl Acad Sci U S A 98, 8065-8070. 
Hua, Y., Sinha, R., Martineau, M., Kahms, M., and Klingauf, J. (2010). A common origin of synaptic vesicles undergoing evoked and spontaneous fusion. Nat Neurosci 13, 1451-1453.

Jackson, M.B., and Chapman, E.R. (2008). The fusion pores of $\mathrm{Ca}(2+)$-triggered exocytosis. Nat Struct Mol Biol 15, 684-689.

Jahn, R. (2004). Principles of exocytosis and membrane fusion. Ann N Y Acad Sci 1014, 170-178.

Jahn, R., Lang, T., and Sudhof, T.C. (2003). Membrane fusion. Cell 112, 519-533.

Jahn, R., and Scheller, R.H. (2006). SNAREs--engines for membrane fusion. Nat Rev Mol Cell Biol 7, 631-643.

Ji, W., Xu, P., Li, Z., Lu, J., Liu, L., Zhan, Y., Chen, Y., Hille, B., Xu, T., and Chen, L. (2008). Functional stoichiometry of the unitary calcium-release-activated calcium channel. Proc Natl Acad Sci U S A 105, 13668-13673.

Jockusch, W.J., Speidel, D., Sigler, A., Sorensen, J.B., Varoqueaux, F., Rhee, J.S., and Brose, N. (2007). CAPS-1 and CAPS-2 are essential synaptic vesicle priming proteins. Cell 131, 796-808.

Joo, C., Balci, H., Ishitsuka, Y., Buranachai, C., and Ha, T. (2008). Advances in single-molecule fluorescence methods for molecular biology. Annu Rev Biochem 77, 51-76.

Karatekin, E., Di Giovanni, J., Iborra, C., Coleman, J., O'Shaughnessy, B., Seagar, M., and Rothman, J.E. A fast, single-vesicle fusion assay mimics physiological SNARE requirements. Proc Natl Acad Sci U S A $107,3517-3521$.

Karatekin, E., Di Giovanni, J., Iborra, C., Coleman, J., O'Shaughnessy, B., Seagar, M., and Rothman, J.E. (2010). A fast, single-vesicle fusion assay mimics physiological SNARE requirements. Proc Natl Acad Sci U S A 107, 3517-3521.

Katz, B., and Miledi, R. (1965). Release of acetylcholine from a nerve terminal by electric pulses of variable strength and duration. Nature 207, 1097-1098.

Katz, B., and Miledi, R. (1967). Ionic requirements of synaptic transmitter release. Nature 215, 651.

Katz, B., and Miledi, R. (1969). Spontaneous and evoked activity of motor nerve endings in calcium Ringer. J Physiol 203, 689-706.

Kawasaki, F., Hazen, M., and Ordway, R.W. (2000). Fast synaptic fatigue in shibire mutants reveals a rapid requirement for dynamin in synaptic vesicle membrane trafficking. Nat Neurosci 3, 859-860.

Kawasaki, F., Mattiuz, A.M., and Ordway, R.W. (1998). Synaptic physiology and ultrastructure in comatose mutants define an in vivo role for NSF in neurotransmitter release. J Neurosci 18, 1024110249.

Keller, J.E., Cai, F., and Neale, E.A. (2004). Uptake of botulinum neurotoxin into cultured neurons. Biochemistry 43, 526-532.

Kim, S.H., and Ryan, T.A. (2009). Synaptic vesicle recycling at CNS snapses without AP-2. J Neurosci 29, 3865-3874.

Kiseleva, E.V., Storozhevykh, T.P., Pinelis, V.G., Gluza, E., and Strukova, S.M. (2004). Role of thrombin in activation of neurons in rat hippocampus. Bull Exp Biol Med 137, 453-456.

Klingauf, J., Kavalali, E.T., and Tsien, R.W. (1998). Kinetics and regulation of fast endocytosis at hippocampal synapses. Nature 394, 581-585.

Kloepper, T.H., Kienle, C.N., and Fasshauer, D. (2007). An elaborate classification of SNARE proteins sheds light on the conservation of the eukaryotic endomembrane system. Mol Biol Cell 18, 3463-3471.

Klyachko, V.A., and Jackson, M.B. (2002). Capacitance steps and fusion pores of small and largedense-core vesicles in nerve terminals. Nature 418, 89-92.

Knowles, M.K., Barg, S., Wan, L., Midorikawa, M., Chen, X., and Almers, W. (2010). Single secretory granules of live cells recruit syntaxin-1 and synaptosomal associated protein 25 (SNAP-25) in large copy numbers. Proc Natl Acad Sci U S A 107, 20810-20815.

Koenig, J.H., and Ikeda, K. (1989). Disappearance and reformation of synaptic vesicle membrane upon transmitter release observed under reversible blockage of membrane retrieval. J Neurosci 9, 3844-3860.

Koenig, J.H., and Ikeda, K. (1996). Synaptic vesicles have two distinct recycling pathways. J Cell Biol 135, 797-808. 
Koenig, J.H., Yamaoka, K., and Ikeda, K. (1998). Omega images at the active zone may be endocytotic rather than exocytotic: implications for the vesicle hypothesis of transmitter release. Proc Natl Acad Sci U S A 95, 12677-12682.

Koh, T.W., and Bellen, H.J. (2003). Synaptotagmin I, a Ca2+ sensor for neurotransmitter release. Trends Neurosci 26, 413-422.

Kohout, S.C., Ulbrich, M.H., Bell, S.C., and Isacoff, E.Y. (2008). Subunit organization and functional transitions in Ci-VSP. Nat Struct Mol Biol 15, 106-108.

Kozlovsky, Y., and Kozlov, M.M. (2002). Stalk model of membrane fusion: solution of energy crisis. Biophys J 82, 882-895.

Kraszewski, K., Mundigl, O., Daniell, L., Verderio, C., Matteoli, M., and De Camilli, P. (1995). Synaptic vesicle dynamics in living cultured hippocampal neurons visualized with CY3-conjugated antibodies directed against the lumenal domain of synaptotagmin. J Neurosci 15, 4328-4342.

Kubitscheck, U., Kuckmann, O., Kues, T., and Peters, R. (2000). Imaging and tracking of single GFP molecules in solution. Biophys J 78, 2170-2179.

Landis, D.M. (1988). Membrane and cytoplasmic structure at synaptic junctions in the mammalian central nervous system. J Electron Microsc Tech 10, 129-151.

Leake, M.C., Chandler, J.H., Wadhams, G.H., Bai, F., Berry, R.M., and Armitage, J.P. (2006). Stoichiometry and turnover in single, functioning membrane protein complexes. Nature 443, 355-358.

Lemke, E.A., and Klingauf, J. (2005). Single synaptic vesicle tracking in individual hippocampal boutons at rest and during synaptic activity. J Neurosci 25, 11034-11044.

Li, F., Pincet, F., Perez, E., Eng, W.S., Melia, T.J., Rothman, J.E., and Tareste, D. (2007). Energetics and dynamics of SNAREpin folding across lipid bilayers. Nat Struct Mol Biol 14, 890-896.

Lin, R.C., and Scheller, R.H. (1997). Structural organization of the synaptic exocytosis core complex. Neuron 19, 1087-1094.

Lindau, M., and Almers, W. (1995). Structure and function of fusion pores in exocytosis and ectoplasmic membrane fusion. Curr Opin Cell Biol 7, 509-517.

Lindau, M., and Alvarez de Toledo, G. (2003). The fusion pore. Biochim Biophys Acta 1641, 167-173.

Lippincott-Schwartz, J., Presley, J.F., Zaal, K.J., Hirschberg, K., Miller, C.D., and Ellenberg, J. (1999). Monitoring the dynamics and mobility of membrane proteins tagged with green fluorescent protein. Methods Cell Biol 58, 261-281.

Lisman, J.E., Raghavachari, S., and Tsien, R.W. (2007). The sequence of events that underlie quantal transmission at central glutamatergic synapses. Nat Rev Neurosci 8, 597-609.

Liu, H., Dean, C., Arthur, C.P., Dong, M., and Chapman, E.R. (2009). Autapses and networks of hippocampal neurons exhibit distinct synaptic transmission phenotypes in the absence of synaptotagmin I. J Neurosci 29, 7395-7403.

Lou, X., Scheuss, V., and Schneggenburger, R. (2005). Allosteric modulation of the presynaptic Ca2+ sensor for vesicle fusion. Nature 435, 497-501.

Martens, H., Weston, M.C., Boulland, J.L., Gronborg, M., Grosche, J., Kacza, J., Hoffmann, A., Matteoli, M., Takamori, S., Harkany, T., et al. (2008). Unique luminal localization of VGAT-C terminus allows for selective labeling of active cortical GABAergic synapses. J Neurosci 28, 13125-13131.

Martens, S., Kozlov, M.M., and McMahon, H.T. (2007). How synaptotagmin promotes membrane fusion. Science 316, 1205-1208.

Martens, S., and McMahon, H.T. (2008). Mechanisms of membrane fusion: disparate players and common principles. Nat Rev Mol Cell Biol 9, 543-556.

Martin, T.F. (2000). Racing lipid rafts for synaptic-vesicle formation. Nat Cell Biol 2, E9-11.

Matteoli, M., Takei, K., Perin, M.S., Sudhof, T.C., and De Camilli, P. (1992). Exo-endocytotic recycling of synaptic vesicles in developing processes of cultured hippocampal neurons. J Cell Biol 117, 849-861.

McMahon, H.T., Ushkaryov, Y.A., Edelmann, L., Link, E., Binz, T., Niemann, H., Jahn, R., and Sudhof, T.C. (1993). Cellubrevin is a ubiquitous tetanus-toxin substrate homologous to a putative synaptic vesicle fusion protein. Nature 364, 346-349. 
Meinrenken, C.J., Borst, J.G., and Sakmann, B. (2002). Calcium secretion coupling at calyx of held governed by nonuniform channel-vesicle topography. J Neurosci 22, 1648-1667.

Meinrenken, C.J., Borst, J.G., and Sakmann, B. (2003). Local routes revisited: the space and time dependence of the $\mathrm{Ca} 2+$ signal for phasic transmitter release at the rat calyx of Held. J Physiol 547, 665689.

Merrifield, C.J., Feldman, M.E., Wan, L., and Almers, W. (2002). Imaging actin and dynamin recruitment during invagination of single clathrin-coated pits. Nat Cell Biol 4, 691-698.

Miesenbock, G., De Angelis, D.A., and Rothman, J.E. (1998). Visualizing secretion and synaptic transmission with pH-sensitive green fluorescent proteins. Nature 394, 192-195.

Miller, T.M., and Heuser, J.E. (1984). Endocytosis of synaptic vesicle membrane at the frog neuromuscular junction. J Cell Biol 98, 685-698.

Mohrmann, R., de Wit, H., Verhage, M., Neher, E., and Sorensen, J.B. (2010). Fast vesicle fusion in living cells requires at least three SNARE complexes. Science 330, 502-505.

Montecucco, C., Schiavo, G., and Pantano, S. (2005). SNARE complexes and neuroexocytosis: how many, how close? Trends Biochem Sci 30, 367-372.

Mueller, V.J., Wienisch, M., Nehring, R.B., and Klingauf, J. (2004). Monitoring clathrin-mediated endocytosis during synaptic activity. J Neurosci 24, 2004-2012.

Murthy, V.N., and Stevens, C.F. (1998). Synaptic vesicles retain their identity through the endocytic cycle. Nature 392, 497-501.

Murthy, V.N., and Stevens, C.F. (1999). Reversal of synaptic vesicle docking at central synapses. Nat Neurosci 2, 503-507.

Mutch, S.A., Kensel-Hammes, P., Gadd, J.C., Fujimoto, B.S., Allen, R.W., Schiro, P.G., Lorenz, R.M., Kuyper, C.L., Kuo, J.S., Bajjalieh, S.M., et al. (2011). Protein quantification at the single vesicle level reveals that a subset of synaptic vesicle proteins are trafficked with high precision. J Neurosci 31, 14611470.

Nakajo, K., Ulbrich, M.H., Kubo, Y., and Isacoff, E.Y. (2010). Stoichiometry of the KCNQ1 - KCNE1 ion channel complex. Proc Natl Acad Sci U S A 107, 18862-18867.

Neale, E.A., Bowers, L.M., Jia, M., Bateman, K.E., and Williamson, L.C. (1999). Botulinum neurotoxin A blocks synaptic vesicle exocytosis but not endocytosis at the nerve terminal. J Cell Biol 147, 1249-1260.

Neher, E., and Marty, A. (1982). Discrete changes of cell membrane capacitance observed under conditions of enhanced secretion in bovine adrenal chromaffin cells. Proc Natl Acad Sci U S A 79, 67126716.

Nicholson-Tomishima, K., and Ryan, T.A. (2004). Kinetic efficiency of endocytosis at mammalian CNS synapses requires synaptotagmin I. Proc Natl Acad Sci U S A 101, 16648-16652.

Opazo, F., Punge, A., Buckers, J., Hoopmann, P., Kastrup, L., Hell, S.W., and Rizzoli, S.O. (2010). Limited intermixing of synaptic vesicle components upon vesicle recycling. Traffic 11, 800-812.

Paillart, C., Li, J., Matthews, G., and Sterling, P. (2003). Endocytosis and vesicle recycling at a ribbon synapse. J Neurosci 23, 4092-4099.

Palm, G.J., Zdanov, A., Gaitanaris, G.A., Stauber, R., Pavlakis, G.N., and Wlodawer, A. (1997). The structural basis for spectral variations in green fluorescent protein. Nat Struct Biol 4, 361-365.

Pang, Z.P., Sun, J., Rizo, J., Maximov, A., and Sudhof, T.C. (2006). Genetic analysis of synaptotagmin 2 in spontaneous and Ca2+-triggered neurotransmitter release. EMBO J 25, 2039-2050.

Parks, T.D., Howard, E.D., Wolpert, T.J., Arp, D.J., and Dougherty, W.G. (1995). Expression and purification of a recombinant tobacco etch virus Nla proteinase: biochemical analyses of the full-length and a naturally occurring truncated proteinase form. Virology 210, 194-201.

Patterson, G.H., Knobel, S.M., Sharif, W.D., Kain, S.R., and Piston, D.W. (1997). Use of the green fluorescent protein and its mutants in quantitative fluorescence microscopy. Biophys J 73, 2782-2790.

Pearse, B.M. (1976). Clathrin: a unique protein associated with intracellular transfer of membrane by coated vesicles. Proc Natl Acad Sci U S A 73, 1255-1259.

Perrais, D., and Merrifield, C.J. (2005). Dynamics of endocytic vesicle creation. Dev Cell 9, 581-592. 
Pierce, D.W., Hom-Booher, N., and Vale, R.D. (1997). Imaging individual green fluorescent proteins. Nature 388, 338.

Piston, D.W., Patterson, G.H., and Knobel, S.M. (1999). Quantitative imaging of the green fluorescent protein (GFP). Methods Cell Biol 58, 31-48.

Pobbati, A.V., Stein, A., and Fasshauer, D. (2006). N- to C-terminal SNARE complex assembly promotes rapid membrane fusion. Science 313, 673-676.

Poirier, M.A., Hao, J.C., Malkus, P.N., Chan, C., Moore, M.F., King, D.S., and Bennett, M.K. (1998). Protease resistance of syntaxin.SNAP-25.VAMP complexes. Implications for assembly and structure. J Biol Chem 273, 11370-11377.

Pyle, J.L., Kavalali, E.T., Piedras-Renteria, E.S., and Tsien, R.W. (2000). Rapid reuse of readily releasable pool vesicles at hippocampal synapses. Neuron 28, 221-231.

Reese, C., Heise, F., and Mayer, A. (2005). Trans-SNARE pairing can precede a hemifusion intermediate in intracellular membrane fusion. Nature 436, 410-414.

Reimer, R.J., Fon, E.A., and Edwards, R.H. (1998). Vesicular neurotransmitter transport and the presynaptic regulation of quantal size. Curr Opin Neurobiol 8, 405-412.

Rettig, J., and Neher, E. (2002). Emerging roles of presynaptic proteins in Ca++-triggered exocytosis. Science 298, 781-785.

Richards, D.A., Guatimosim, C., and Betz, W.J. (2000). Two endocytic recycling routes selectively fill two vesicle pools in frog motor nerve terminals. Neuron 27, 551-559.

Rizo, J., and Rosenmund, C. (2008). Synaptic vesicle fusion. Nat Struct Mol Biol 15, 665-674.

Rizzoli, S.O., and Jahn, R. (2007). Kiss-and-run, collapse and 'readily retrievable' vesicles. Traffic 8 , 1137-1144.

Roos, J., and Kelly, R.B. (1999). The endocytic machinery in nerve terminals surrounds sites of exocytosis. Curr Biol 9, 1411-1414.

Rosenboom, H., and Lindau, M. (1994). Exo-endocytosis and closing of the fission pore during endocytosis in single pituitary nerve terminals internally perfused with high calcium concentrations. Proc Natl Acad Sci U S A 91, 5267-5271.

Rosenmund, C., Rettig, J., and Brose, N. (2003). Molecular mechanisms of active zone function. Curr Opin Neurobiol 13, 509-519.

Rosenmund, C., and Stevens, C.F. (1996). Definition of the readily releasable pool of vesicles at hippocampal synapses. Neuron 16, 1197-1207.

Ryan, T.A. (2006). A pre-synaptic to-do list for coupling exocytosis to endocytosis. Curr Opin Cell Biol 18, 416-421.

Sampo, B., Kaech, S., Kunz, S., and Banker, G. (2003). Two distinct mechanisms target membrane proteins to the axonal surface. Neuron 37, 611-624.

Sankaranarayanan, S., Atluri, P.P., and Ryan, T.A. (2003). Actin has a molecular scaffolding, not propulsive, role in presynaptic function. Nat Neurosci 6, 127-135.

Sankaranarayanan, S., De Angelis, D., Rothman, J.E., and Ryan, T.A. (2000). The use of pHluorins for optical measurements of presynaptic activity. Biophys J 79, 2199-2208.

Sankaranarayanan, S., and Ryan, T.A. (2000). Real-time measurements of vesicle-SNARE recycling in synapses of the central nervous system. Nat Cell Biol 2, 197-204.

Sankaranarayanan, S., and Ryan, T.A. (2001). Calcium accelerates endocytosis of vSNAREs at hippocampal synapses. Nat Neurosci 4, 129-136.

Sara, Y., Virmani, T., Deak, F., Liu, X., and Kavalali, E.T. (2005). An isolated pool of vesicles recycles at rest and drives spontaneous neurotransmission. Neuron 45, 563-573.

Schiavo, G., Matteoli, M., and Montecucco, C. (2000). Neurotoxins affecting neuroexocytosis. Physiol Rev 80, 717-766.

Schikorski, T., and Stevens, C.F. (1997). Quantitative ultrastructural analysis of hippocampal excitatory synapses. J Neurosci 17, 5858-5867. 
Schmidt, R., Wurm, C.A., Jakobs, S., Engelhardt, J., Egner, A., and Hell, S.W. (2008). Spherical nanosized focal spot unravels the interior of cells. Nat Methods 5, 539-544.

Schneggenburger, R., and Neher, E. (2000). Intracellular calcium dependence of transmitter release rates at a fast central synapse. Nature 406, 889-893.

Schoch, S., Deak, F., Konigstorfer, A., Mozhayeva, M., Sara, Y., Sudhof, T.C., and Kavalali, E.T. (2001). SNARE function analyzed in synaptobrevin/VAMP knockout mice. Science 294, 1117-1122.

Schoch, S., and Gundelfinger, E.D. (2006). Molecular organization of the presynaptic active zone. Cell Tissue Res 326, 379-391.

Schweizer, F.E., and Ryan, T.A. (2006). The synaptic vesicle: cycle of exocytosis and endocytosis. Curr Opin Neurobiol 16, 298-304.

Siksou, L., Varoqueaux, F., Pascual, O., Triller, A., Brose, N., and Marty, S. (2009). A common molecular basis for membrane docking and functional priming of synaptic vesicles. Eur J Neurosci 30, 49-56.

Simpson, L.L. (2004). Identification of the major steps in botulinum toxin action. Annu Rev Pharmacol Toxicol 44, 167-193.

Slepnev, V.I., and De Camilli, P. (2000). Accessory factors in clathrin-dependent synaptic vesicle endocytosis. Nat Rev Neurosci 1, 161-172.

Smith-Swintosky, V.L., Cheo-Isaacs, C.T., D'Andrea, M.R., Santulli, R.J., Darrow, A.L., and AndradeGordon, P. (1997). Protease-activated receptor-2 (PAR-2) is present in the rat hippocampus and is associated with neurodegeneration. J Neurochem 69, 1890-1896.

Smith, S.M., Renden, R., and von Gersdorff, H. (2008). Synaptic vesicle endocytosis: fast and slow modes of membrane retrieval. Trends Neurosci 31, 559-568.

Sollner, T., Bennett, M.K., Whiteheart, S.W., Scheller, R.H., and Rothman, J.E. (1993a). A protein assembly-disassembly pathway in vitro that may correspond to sequential steps of synaptic vesicle docking, activation, and fusion. Cell 75, 409-418.

Sollner, T., Whiteheart, S.W., Brunner, M., Erdjument-Bromage, H., Geromanos, S., Tempst, P., and Rothman, J.E. (1993b). SNAP receptors implicated in vesicle targeting and fusion. Nature 362, 318-324.

Sorensen, J.B. (2004). Formation, stabilisation and fusion of the readily releasable pool of secretory vesicles. Pflugers Arch 448, 347-362.

Sorensen, J.B., Nagy, G., Varoqueaux, F., Nehring, R.B., Brose, N., Wilson, M.C., and Neher, E. (2003). Differential control of the releasable vesicle pools by SNAP-25 splice variants and SNAP-23. Cell 114, 75-86.

Stevens, C.F., and Sullivan, J.M. (2003). The synaptotagmin C2A domain is part of the calcium sensor controlling fast synaptic transmission. Neuron 39, 299-308.

Stewart, B.A., Mohtashami, M., Trimble, W.S., and Boulianne, G.L. (2000). SNARE proteins contribute to calcium cooperativity of synaptic transmission. Proc Natl Acad Sci U S A 97, 13955-13960.

Steyer, J.A., Horstmann, H., and Almers, W. (1997). Transport, docking and exocytosis of single secretory granules in live chromaffin cells. Nature $388,474-478$.

Sudhof, T.C. (1995). The synaptic vesicle cycle: a cascade of protein-protein interactions. Nature 375, 645-653.

Sudhof, T.C. (2004). The synaptic vesicle cycle. Annu Rev Neurosci 27, 509-547.

Sudhof, T.C., and Rothman, J.E. (2009). Membrane fusion: grappling with SNARE and SM proteins. Science 323, 474-477.

Sugiyama, Y., Kawabata, I., Sobue, K., and Okabe, S. (2005). Determination of absolute protein numbers in single synapses by a GFP-based calibration technique. Nat Methods 2, 677-684.

Sutton, R.B., Fasshauer, D., Jahn, R., and Brunger, A.T. (1998). Crystal structure of a SNARE complex involved in synaptic exocytosis at 2.4 A resolution. Nature 395, 347-353.

Tabares, L., Ruiz, R., Linares-Clemente, P., Gaffield, M.A., Alvarez de Toledo, G., Fernandez-Chacon, R., and Betz, W.J. (2007). Monitoring synaptic function at the neuromuscular junction of a mouse expressing synaptopHluorin. J Neurosci 27, 5422-5430.

Takamori, S., Holt, M., Stenius, K., Lemke, E.A., Gronborg, M., Riedel, D., Urlaub, H., Schenck, S., Brugger, B., Ringler, P., et al. (2006). Molecular anatomy of a trafficking organelle. Cell 127, 831-846. 
Takei, K., and Haucke, V. (2001). Clathrin-mediated endocytosis: membrane factors pull the trigger. Trends Cell Biol 11, 385-391.

Takei, K., Mundigl, O., Daniell, L., and De Camilli, P. (1996). The synaptic vesicle cycle: a single vesicle budding step involving clathrin and dynamin. J Cell Biol 133, 1237-1250.

Taubenblatt, P., Dedieu, J.C., Gulik-Krzywicki, T., and Morel, N. (1999). VAMP (synaptobrevin) is present in the plasma membrane of nerve terminals. J Cell Sci 112 ( Pt 20), 3559-3567.

Teng, H., Cole, J.C., Roberts, R.L., and Wilkinson, R.S. (1999). Endocytic active zones: hot spots for endocytosis in vertebrate neuromuscular terminals. J Neurosci 19, 4855-4866.

Teng, H., and Wilkinson, R.S. (2000). Clathrin-mediated endocytosis near active zones in snake motor boutons. J Neurosci 20, 7986-7993.

Thiele, C., Hannah, M.J., Fahrenholz, F., and Huttner, W.B. (2000). Cholesterol binds to synaptophysin and is required for biogenesis of synaptic vesicles. Nat Cell Biol 2, 42-49.

Thomas, P., Lee, A.K., Wong, J.G., and Almers, W. (1994). A triggered mechanism retrieves membrane in seconds after $\mathrm{Ca}(2+)$-stimulated exocytosis in single pituitary cells. J Cell Biol 124, 667-675.

Threadgill, R., Bobb, K., and Ghosh, A. (1997). Regulation of dendritic growth and remodeling by Rho, Rac, and Cdc42. Neuron 19, 625-634.

Tokumaru, H., Umayahara, K., Pellegrini, L.L., Ishizuka, T., Saisu, H., Betz, H., Augustine, G.J., and Abe, T. (2001). SNARE complex oligomerization by synaphin/complexin is essential for synaptic vesicle exocytosis. Cell 104, 421-432.

Tombola, F., Ulbrich, M.H., and Isacoff, E.Y. (2008). The voltage-gated proton channel Hv1 has two pores, each controlled by one voltage sensor. Neuron $58,546-556$.

Trimble, W.S., Gray, T.S., Elferink, L.A., Wilson, M.C., and Scheller, R.H. (1990). Distinct patterns of expression of two VAMP genes within the rat brain. J Neurosci 10, 1380-1387.

Tsien, R.Y. (1998). The green fluorescent protein. Annu Rev Biochem 67, 509-544.

Ulbrich, M.H., and Isacoff, E.Y. (2007). Subunit counting in membrane-bound proteins. Nat Methods 4 , 319-321.

Vale, R.D. (2008). Microscopes for fluorimeters: the era of single molecule measurements. Cell 135 , 779-785.

Vale, R.D., Funatsu, T., Pierce, D.W., Romberg, L., Harada, Y., and Yanagida, T. (1996). Direct observation of single kinesin molecules moving along microtubules. Nature 380, 451-453.

van den Bogaart, G., Holt, M.G., Bunt, G., Riedel, D., Wouters, F.S., and Jahn, R. One SNARE complex is sufficient for membrane fusion. Nat Struct Mol Biol 17, 358-364.

van den Bogaart, G., Holt, M.G., Bunt, G., Riedel, D., Wouters, F.S., and Jahn, R. (2010). One SNARE complex is sufficient for membrane fusion. Nat Struct Mol Biol 17, 358-364.

Vanden Berghe, P., and Klingauf, J. (2006). Synaptic vesicles in rat hippocampal boutons recycle to different pools in a use-dependent fashion. J Physiol 572, 707-720.

Verhage, M., and Sorensen, J.B. (2008). Vesicle docking in regulated exocytosis. Traffic 9, 1414-1424.

Verstreken, P., Koh, T.W., Schulze, K.L., Zhai, R.G., Hiesinger, P.R., Zhou, Y., Mehta, S.Q., Cao, Y., Roos, J., and Bellen, H.J. (2003). Synaptojanin is recruited by endophilin to promote synaptic vesicle uncoating. Neuron 40, 733-748.

Voets, T., Toonen, R.F., Brian, E.C., de Wit, H., Moser, T., Rettig, J., Sudhof, T.C., Neher, E., and Verhage, M. (2001). Munc18-1 promotes large dense-core vesicle docking. Neuron 31, 581-591.

Voglmaier, S.M., and Edwards, R.H. (2007). Do different endocytic pathways make different synaptic vesicles? Curr Opin Neurobiol 17, 374-380.

Wadel, K., Neher, E., and Sakaba, T. (2007). The coupling between synaptic vesicles and Ca2+ channels determines fast neurotransmitter release. Neuron 53, 563-575.

Weber, T., Zemelman, B.V., McNew, J.A., Westermann, B., Gmachl, M., Parlati, F., Sollner, T.H., and Rothman, J.E. (1998). SNAREpins: minimal machinery for membrane fusion. Cell 92, 759-772.

Weimer, R.M., Richmond, J.E., Davis, W.S., Hadwiger, G., Nonet, M.L., and Jorgensen, E.M. (2003). Defects in synaptic vesicle docking in unc-18 mutants. Nat Neurosci 6, 1023-1030. 
Wiederhold, K., and Fasshauer, D. (2009). Is assembly of the SNARE complex enough to fuel membrane fusion? J Biol Chem 284, 13143-13152.

Wienisch, M., and Klingauf, J. (2006). Vesicular proteins exocytosed and subsequently retrieved by compensatory endocytosis are nonidentical. Nat Neurosci 9, 1019-1027.

Willig, K.I., Rizzoli, S.O., Westphal, V., Jahn, R., and Hell, S.W. (2006). STED microscopy reveals that synaptotagmin remains clustered after synaptic vesicle exocytosis. Nature 440, 935-939.

Wu, L.G., Ryan, T.A., and Lagnado, L. (2007). Modes of vesicle retrieval at ribbon synapses, calyx-type synapses, and small central synapses. J Neurosci 27, 11793-11802.

Wu, W., and Wu, L.G. (2007). Rapid bulk endocytosis and its kinetics of fission pore closure at a central synapse. Proc Natl Acad Sci U S A 104, 10234-10239.

Wu, X.S., McNeil, B.D., Xu, J., Fan, J., Xue, L., Melicoff, E., Adachi, R., Bai, L., and Wu, L.G. (2009). $\mathrm{Ca}(2+)$ and calmodulin initiate all forms of endocytosis during depolarization at a nerve terminal. Nat Neurosci 12, 1003-1010.

$\mathrm{Xu}$, J., Mashimo, T., and Sudhof, T.C. (2007). Synaptotagmin-1, -2, and -9: $\mathrm{Ca}(2+)$ sensors for fast release that specify distinct presynaptic properties in subsets of neurons. Neuron 54, 567-581.

Xu, Y., Zhang, F., Su, Z., McNew, J.A., and Shin, Y.K. (2005). Hemifusion in SNARE-mediated membrane fusion. Nat Struct Mol Biol 12, 417-422.

Yao, C.K., Lin, Y.Q., Ly, C.V., Ohyama, T., Haueter, C.M., Moiseenkova-Bell, V.Y., Wensel, T.G., and Bellen, H.J. (2009). A synaptic vesicle-associated $\mathrm{Ca} 2+$ channel promotes endocytosis and couples exocytosis to endocytosis. Cell 138, 947-960.

Zhang, Q., Cao, Y.Q., and Tsien, R.W. (2007). Quantum dots provide an optical signal specific to full collapse fusion of synaptic vesicles. Proc Natl Acad Sci U S A 104, 17843-17848.

Zhang, Q., Li, Y., and Tsien, R.W. (2009). The dynamic control of kiss-and-run and vesicular reuse probed with single nanoparticles. Science 323, 1448-1453.

Zhu, Y., Xu, J., and Heinemann, S.F. (2009). Two pathways of synaptic vesicle retrieval revealed by single-vesicle imaging. Neuron 61, 397-411. 


\section{ABBREVIATIONS}

\begin{tabular}{|c|c|}
\hline AP & Action Potential \\
\hline AP-1, AP-2 & Adaptor protein-1, 2 \\
\hline a.u. & Arbitrary units \\
\hline$A Z$ & Active zone \\
\hline BBS & BBS (N, N-bis[2-hydroxyethyl]-2-aminoethanesulfonic acid \\
\hline BoNT/A & Botulinum neurotoxin serotype $A$ \\
\hline CAZ & Cytoplasmic matrix of active zone \\
\hline Ceb & Cellubrevin \\
\hline CME & Clathrin-mediated endocytosis \\
\hline CNQX & 6-cyano-7-nitroquinoxaline-2,3-dione \\
\hline CNS & Central nervous system \\
\hline $\mathrm{D}, \mathrm{L}-\mathrm{APV}$ & D-amino-5-phosphonovaleric acid \\
\hline EGTA & Ethylene glycol-bis(2-aminoethylether)-N,N,N',N'-tetraacetic acid \\
\hline EM & Electron microscopy \\
\hline EM-CCD & Electron multiplying charged coupled device \\
\hline EPSC & Excitatory postsynaptic current \\
\hline DKO & Double knock-out \\
\hline DIV & Days in vitro \\
\hline EDTA & Ethylenediaminetetraaceticacid \\
\hline EGTA & Ethylene glycol-bis(2-aminoethylether)-N,N,N',N'-tetraacetic acid \\
\hline E18 & Embryonic day 18 \\
\hline FBS & Fetal bovine serum \\
\hline GABA & Y-amino-butyric acid \\
\hline GFP & Green fluorescent protein \\
\hline $\mathrm{kDa}$ & Kilo Dalton \\
\hline $\mathrm{KO}$ & Knock-out \\
\hline MES & 2-[N-morpholino]ethane sulphonic acid] \\
\hline NA & Numerical aperture \\
\hline NSF & $\mathrm{N}$-ethylmaleimide-sensitive factor \\
\hline NT & Neurotransmitter \\
\hline $\mathrm{PC} 12$ & cell line derived from PheoChromocytoma of the rat adrenal medulla \\
\hline PFA & Paraformaldehyde \\
\hline $\mathrm{pHI}$ & pHluorin \\
\hline $\mathrm{P}_{\mathrm{r}}$ & Release probability \\
\hline PSD & Post synaptic density \\
\hline q & Quantal size \\
\hline
\end{tabular}


$\mathrm{RIM} 1 / 2$

$\mathrm{ROI}$

RRP

RRetP

s.d.

s.e.m.

SNAP

SNAP-25

SNARE

$\mathrm{SpH}$

STED

Syb2

Syp1

Syt1

Syx $1 A$

SV

TeNT

TEV

TIRFM

TMD

TTX

vGlut

VGAT

WT
Rab3-interacting molecule 1/2

Region of interest

Readily releasable pool

Readily retrievable pool

Standard deviation

Standard error of mean

Soluble NSF attachment protein

Synaptosomal associated protein of $25 \mathrm{kDa}$

SNAP receptor

Synaptobrevin2-pHluorin

Stimulated emission depletion

Synaptobrevin2

Synaptophysin 1

Synaptotagmin 1

Syntaxin-1A

Synaptic vesicle

Tetanus neurotoxin

Tobacco-etch-virus

Total internal reflection fluorescence microscopy

Transmembrane domain

Tetrodotoxin

Vesicle glutamate transporter

Vesicle GABA transporter

Wild type 


\section{ACKNOWLEDGMENTS}

First of all I would like to thank my PhD supervisor Prof. Jürgen Klingauf for giving me the opportunity to work in his group, for exposing me to the exciting field of exo- and endocytosis, for the motivation to undertake challenging projects and at the same time for being a very patient mentor. His incredible urge to know the outcome of expeiments, his openness to new ideas, the liberty to try unconventional experiments and the high level of enthusiasm about the results has made $\mathrm{my} \mathrm{PhD}$ research a rich and a great learning experience.

I am grateful to the members of my thesis committee, Prof. Erwin Neher for his continuous support and valuable advice on my project and Prof. Walter Stühmer for his suggestions during the thesis committee meetings.

I would like to acknowledge members of the Dept. of Neurobiology for a fruitful collaboration; Dr. Saheeb Ahmed for his help with biochemistry and Prof. Reinhard Jahn for his critical suggestions and for sharing the same level of enthusiasm, as we did, about the SNARE complex work. In addition, I would also like to acknowledge Dr. Matthew Holt for introducing me to the field of membrane fusion and Dr. Geert van den Bogaart for his feedback and fruitful discussions.

I would like to express my gratitude to the IMPRS MSc/PhD Neuroscience program for giving me the opportunity to be a part of the excellent neuroscience research community in Göttingen. A special thanks to the co-ordination team of Prof. Michael Hörner and Ms. Sandra Drube, for their endearing support, in all respects over the years, which made life in Göttingen a remarkable experience.

I would like to thank the past and present members of our research group MOST (microscopy of synaptic transmission) for creating a lively working environment both intellectually and otherwise. I thank Dr. Teja Groemer for his help and advice in the initial phase of my PhD, Dr. Cora Thiel for her help with molecular biology and purification of the pHluorin protein, Dr. Yaroslav Tsytsyura for help with the microscope set-ups, Dr. Natalia Glyvuk for kind advice on immunostaining, Daniel Böning for his assistance with the TIRF set-up, Benjamin Wilhelm and Sebastian Richter for their enthusiasm and support. I would like to specially thank my colleague Yunfeng Hua for helping me with the cypHer projects, his programming skills, for constant brainstorming sessions, which resulted in amazing ideas that steered two fruitful projects. Furthermore, I would like to acknowledge Michael Pilot, Ina Herfort and Dirk Reuter for their expert technical assistance. 
I would like to acknowledge all the members of the Dept. of Membrane Biophysics for their support. Thanks to Dr. Takeshi Sakaba for reading my thesis, intense discussions, ideas and advice on my project, to Kun-Han Lin, Chao-Hua Huang and Lijun Yao, for their help and support. I would like to express my gratitude to Dr. Andrew Woehler for his help in statistical analysis and imaging. I would also like to thank Frank Würriehausen for help with computers, Frank Köhne for help with electronics, Irmgard Barteczko for all the administrative support and the members of the animal house facility for maintaining the mouse lines.

I would like to express my gratitude to all my friends for their moral support over the years. My deepest thanks to Mrinalini Hoon for proof-reading my thesis, more importantly for being the cornerstone of my life and for being an endless source of help, motivation and inspiration. Last but not the least I would like to thank my parents and my brother for their love and support all along. 


\section{CURRICULUM VITAE}

\section{Personal details :}

Name :

Date of Birth :

Place of Birth :

Nationality :

Work address :

Email :

\section{Education :}

Aug'02 - May'05

Jul' 05 - Jun'06

Sept'06 - Aug'07

Sept'07 - May'11
Raunak Sinha

12th Feb, 1985

Kolkata, India

Indian

Dept. of Membrane Biophysics

Max-Planck Inst. for Biophysical Chemistry

Am Fassberg 11

37077 Göttingen

rsinha@gwdg.de

BSc (Hons) in Physiology

Calcutta University, Presidency College, Kolkata, India

Junior Research Scholar

Tata Institute of Fundamental Research (TIFR), India

International Max Planck Research School for Neurosciences

MSc/PhD Neuroscience Program, Georg August-University Göttingen,

Germany

$\mathrm{PhD}$ thesis in the research group of Microscopy of Synaptic

Transmission under the supervision of Prof. Jürgen Klingauf at the

Dept. of Membrane Biophysics, Max Planck Institute for Biophysical

Chemistry, Göttingen, Germany 


\section{Congress Contributions :}

Jul'08

N.Glyvuk, Y.Tsytsyura, C.Thiel, R. Sinha, J.Wienands, J.Klingauf. Role of mAbp1 in synaptic vesicle turnover. 6th FENS forum of European Neuroscience, Geneva, Switzerland. (Poster)

Sept'08

R. Sinha, N.Glyvuk, Y.Tsytsyura, C.Thiel, J.Klingauf. Coupling of actin and dynamin by mAbp1 is essential for vesicle turnover and vesicle size regulation. Horizons in molecular biology, 5th International $\mathrm{PhD}$ student symposium, Göttingen, Germany. (Poster)

Oct'09

R. Sinha, J. Klingauf. Two SNARE complexes are sufficient to drive synaptic vesicle fusion. (Poster)

Y. Hua, R. Sinha, C. S. Thiel, H. Martens, E. Neher, J. Klingauf. Readily retrievable pool of synaptic vesicles. (Poster)

Society for Neuroscience Meeting, Chicago, USA

Jul'10

R. Sinha, J. Klingauf. Two SNARE complexes are sufficient for vesicle fusion in central nervous system synapses. Gordon research conference, 'Synaptic Transmission', UNE, Biddeford, USA. (Poster)

Nov'10

R. Sinha, J. Klingauf. Stochiometry of SNARE complexes sufficient for fast calcium-triggered exocytosis in central nervous system synapses. (Talk)

Y. Hua, R. Sinha, E. Neher, J. Klingauf. A novel optical probe to monitor synaptic vesicle recycling. (Poster)

Society for Neuroscience Meeting, San Diego, USA 


\section{PUBLICATION LIST}

Sinha, R., Ahmed, S., Jahn, R., Klingauf, J.

Two SNARE complexes are sufficient for vesicle fusion in central nervous system synapses.

Proc Natl Acad Sci U S A. 2011 Aug 23; 108(34):14318-23.

Hua, Y.*, Sinha, R.*, Thiel, C.S. ${ }^{*}$, Schmidt, R., Hüve, J., Martens, H., Hell, S.W., Egner, A., Klingauf, J.

A readily retrievable pool of synaptic vesicles.

Nat Neurosci. 2011 Jun 12;14(7):833-9.

${ }^{*}$ equal contribution

Hua, Y.*, Sinha, R.*, Martineau, M., Kahms, M., Klingauf, J.

A common origin of synaptic vesicles undergoing evoked and spontaneous fusion.

Nat Neurosci. 2010 Dec;13(12):1451-3.

${ }^{*}$ equal contribution

Suseendranathan, K., Sengupta, K., Rikhy, R., D'Souza, J.S., Kokkanti, M., Kulkarni, M.G., Kamdar, R., Changede, R., Sinha, R., Subramanian, L., Singh, K., Rodrigues, V., Rao. B.J.

Expression pattern of Drosophila translin and behavioral analyses of the mutant.

Eur J Cell Biol. 2007 Mar; 86(3):173-86. 